\title{
Affine modifications and affine hypersurfaces with a very transitive automorphism group
}

\author{
Sh. Kalimant M. Zaidenberg
}

\begin{abstract}
We study a kind of modification of an affine domain which produces another affine domain. First appeared in passing in the basic paper of O. Zariski Zar, it was further considered by E.D. Davis [Da]. In [Ka 1] its geometric counterpart was applied to construct contractible smooth affine varieties non-isomorphic to Euclidean spaces. Here we provide certain conditions (more general then those in Ka 1]) which guarantee preservation of the topology under a modification.

As an application, we show that the group of biregular automorphisms of the affine hypersurface $X \subset \mathbf{C}^{k+2}$ given by the equation $u v=p\left(x_{1}, \ldots, x_{k}\right)$ where $p \in \mathbf{C}\left[x_{1}, \ldots, x_{k}\right]$, acts $m$ - transitively on the smooth part reg $X$ of $X$ for any $m \in \mathbf{N}$. We present examples of such hypersurfaces diffeomorphic to Euclidean spaces.
\end{abstract}

\section{Contents}

Introduction . . . . . . . . . . . . . . . . . 1

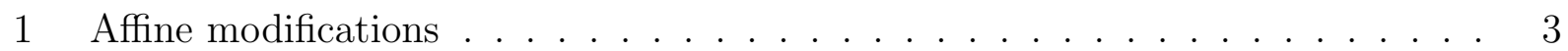

2 The universal property of affine modifications . . . . . . . . . . . 8

3 Topology of affine modifications . . . . . . . . . . . . . . . 12

4 Topology of the hypersurfaces $u v=p\left(x_{1}, \ldots, x_{k}\right) \ldots \ldots \ldots \ldots$

$5 \quad \mathbf{C}_{+}$-actions on the hypersurfaces $u v=p\left(x_{1}, \ldots, x_{k}\right) \ldots \ldots \ldots$

6 Examples of acyclic surfaces in $\mathbf{C}^{3}$ and of smooth contractible 4-folds $u v=$

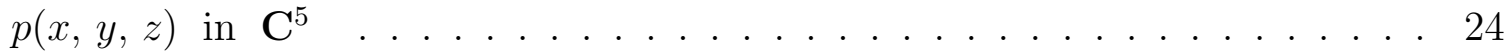

7 On the theorems of Sathaye and Wright . . . . . . . . . . . . 28

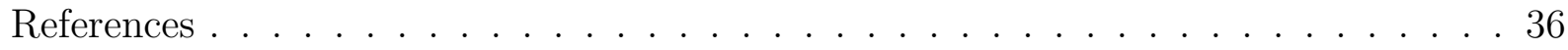

\section{Introduction}

It is well known (and elementary) that for $n>1$ the automorphism group Aut $\mathbf{C}^{n}$ of the affine space $\mathbf{C}^{n}$ (or, which is the same, of the polynomial ring in $n$ variables $\left.\mathbf{C}^{[n]}:=\mathbf{C}\left[x_{1}, \ldots, x_{n}\right]\right)$ acts $m$ - transitively on $\mathbf{C}^{n}$ for any $m \in \mathbf{N}$. That is, the diagonal

${ }^{*}$ Partially supported by the NSA grant MDA904-96-01-0012. 
action of the group Aut $\mathbf{C}^{n}$ on the $m$-th symmetric power $S^{m} \mathbf{C}^{n}$, and even on the $m$ - th Cartesian power $\left(\mathbf{C}^{n}\right)^{m}$, is transitive outside of the diagonals for anyt $m \in \mathbf{N}$. Clearly, every Zariski open subset of the form $\mathbf{C}^{n} \backslash K$ where $K \subset \mathbf{C}^{n}$ is a finite set of points, possesses this property.

Let $V$ be a compact complex space. By a generalized Bochner-Montgomery Theorem Akh, (2.3)], the automorphism group Aut $V$ is a complex Lie group. Therefore, it cannot be $m$-transitive on $V$ for $m>\operatorname{dim}$ Aut $V$. Thus, the question arises:

For which complex manifolds or, at least, for which quasi-projective varieties $X$ the group Aut $X$ is $m$-transitive on $X$ for any $m \in \mathbf{N}$ ?

Or, more restrictively (to exclude the above examples of type $\mathbf{C}^{n} \backslash K$ )

Which affine algebraic varieties possess this property?

In sections 4 and 5 we describe a class of affine hypersurfaces non-homeomorphic, in general, to the affine spaces, with very transitive automorphism groups (see The Transitivity Theorem in sect. 5).

One more remark is in order. One might specify the above problem by asking whether, for $m$ given, there exists an $m$-transitive algebraic group action on $X$. For $m$ sufficiently large with respect to the dimension of $X$, the class of such varieties $X$ seems to be rather poor. Actually, already the affine plane $\mathbf{C}^{2}$ does not admit an $m$ - transitive algebraic group action for $m \geq 3$. Indeed, by the Jung-van der Kulk Theorem [Du, vdK], the group Aut $\mathbf{C}^{2}$ can be represented as the amalgamated product of the affine group Aff $\mathbf{C}^{2}$ and the Jonquière subgroup $J\left(\mathbf{C}^{2}\right) \subset$ Aut $\mathbf{C}^{2}$ of the triangular transformations $(x, y) \longmapsto(x, y+p(x))$ where $p \in \mathbf{C}[x]$. By the theorem of Serre $[\mathrm{Se}]$, any subgroup of finite length $G \subset$ Aut $\mathbf{C}^{2}$ is conjugate either with a subgroup of Aff $\mathbf{C}^{2}$ or with a subgroup of $J\left(\mathbf{C}^{2}\right)$. Since the polynomial $x$ is an invariant of the Jonquière subgroup $J\left(\mathbf{C}^{2}\right)$, in the latter case $G$ possesses a non-constant invariant function, and therefore, it is not even transitive on $\mathbf{C}^{2}$. In the former case, $G$ is at most 2 -transitive; indeed, the collinearity of a triple of points is preserved by the action of the affine group Aff $\mathbf{C}^{2}$. It remains to observe following D. Wright [Wr 1] that any algebraic subgroup $G \subset$ Aut $\mathbf{C}^{2}$ is of finite length.

The proof of Theorem 5.1 on $\infty$ - transitivity of the automorphism group of a smooth hypersurface $X \subset \mathbf{C}^{k+2}, k \geq 2$, given by the equation $u v-p(\bar{x})=0$ goes as follows. This hypersurface $X$ can be naturally presented in two different ways as an affine modification of $\mathbf{C}^{k+1}$ (see below). Due to Corollary 2.2, one can lift to $X$ those automorphisms of the affine space $\mathbf{C}^{k+1}$ which preserve the locus of at least one of these modifications. In such a way we obtain two subgroups $\widehat{G}_{1}$ and $\widehat{G}_{2}$ of the automorphism group Aut $X$. It turns out that the subgroup $\widehat{G}$ of Aut $X$ generated by $\widehat{G}_{1}$ and $\widehat{G}_{2}$ acts $m-$ transitively on $X$ for any $m \in \mathbf{N}$.

While a general hypersurface $X \subset \mathbf{C}^{k+2}$ as above has quite a rich topology, in sections 4 and 6 we give a series of examples of hypersurfaces of this type which are diffeomorphic to the affine space $\mathbf{C}^{k+1}, k \geq 3$. Presumably, they are not, in general, isomorphic to $\mathbf{C}^{k+1}$, and so, they should present new exotic algebraic structures on $\mathbf{C}^{k+1}$ (see e.g. [Za 2]). As well, this would show that the Miyanishi's characterization of the affine 3space $\mathbf{A}^{3}$ Miy cannot be applied to $\mathbf{A}^{n}$ for $n \geq 4$ (see Remark 4.3). But if one

\footnotetext{
${ }^{1}$ See RoRu for an $\infty$ - transitivity property of the group of analytic automorphisms of $\mathbf{C}^{n}$.
} 
of them were isomorphic to $\mathbf{C}^{k+1}$ this would answer in negative alternatively, either to the Zariski Cancellation Problem or to the Abhyankar-Sathaye Embedding Problem (see below).

Sections 1-3 are devoted to a kind of modification which acts on the affine rings producing new affine rings; we call it affine modification. It appeared in passing in the classical Zariski paper [Zar] (see also [Hiro, III.2]), but apparently, the first proper study of this transform was done in $\mathrm{Da}$. A geometric counterpart of the affine modification occurred to be useful in constructing exotic algebraic structures on the affine spaces, as it was done in Ka 1] (see also Za 2 ).

In Corollary 2.2 we lift automorphisms to an affine modification. In section 3 we provide certain conditions (more general then those in [Ka 1]) which guarantee preservation of the topology under a modification.

Recall the Abhyankar-Sathaye Embedding Problem:

Is every closed embedding of $\mathbf{C}^{k}$ into $\mathbf{C}^{n}$ rectifiable, i.e. equivalent to a linear one up to the actions of the automorphisms groups Aut $\mathbf{C}^{k}$ resp. Aut $\mathbf{C}^{n}$ ?

In section 7 we give a generalization of the Sathaye-Wright Theorem [Sat, Wr 2] which guarantees rectifiability of the special embeddings $\mathbf{C}^{2} \hookrightarrow \mathbf{C}^{3}$ given by the equations of the form $f(x, y) z^{n}+g(x, y)=0$. Notice that for $n=1$ such a surface is an affine modification of the affine plane $\mathbf{C}^{2}$ along the divisor $D_{f}=(f)$ with center at the ideal $I=(f, g) \subset \mathbf{C}[x, y]$. More generally, in Theorem 7.2 we show that any smooth acyclic surface $X \subset \mathbf{C}^{3}$ given by the equation $f(x, y) z^{n}+g(x, y)=0$ is isomorphic to $\mathbf{C}^{2}$ (and can be rectified). This is no longer true for $\mathrm{Q}$ - acyclic surfaces; see Example 7.1.

\section{Affine modifications}

Consider a triple $(A, I, f)$ consisting of a commutative ring A with unity, an ideal $I$ of $A$, and an element $f$ of $I$ which is not a zero divisor. We call it a Noetherian triple if $A$ is Noetherian, and an affine triple (over $\mathbf{C}$ ) if $A$ is an affine domain / $\mathbf{C}$, that is, a finitely generated commutative $\mathbf{C}$ - algebra which is a domain. With $t$ being a new symbol, denote by

$$
A[I t]=A \oplus \bigoplus_{n=1}^{\infty}(I t)^{n} \simeq A \oplus I \oplus I^{2} \oplus \ldots=B l_{I}(A)
$$

the blowup algebra, or the Rees algebra [Ei, §5.2], [Va].

Definition 1.1. By the affine modification $\Sigma_{I, f}(A)$ of the ring $A$ along $(f)$ with center $I$ (or, shortly, with the locus $(I, f)$ ) we mean the quotient of the blowup algebra $A[I t]$ by the principal ideal generated by the element $1-f t \in A[I t]$ :

$$
\Sigma_{I, f}(A)=A[I t] /(1-f t) .
$$

When $f$ and $I$ are fixed, without abuse of notation we denote $A^{\prime}=\Sigma_{I, f}(A)$. Clearly, if $(A, I, f)$ is a Noetherian triple, then $A^{\prime}$ is again a commutative Noetherian ring with unity. 
Our purposes in this paper are mainly of geometric naturel. Thereby, we adopt the following

Conventions. Hereafter $(A, I, f)$ is assumed to be an affine triple / C. Besides, we fix two systems of generators $a_{1}, \ldots, a_{r}$ of the algebra $A$ resp. $b_{0}=f, b_{1}, \ldots, b_{s}$ of the ideal $I$. Denoting $\mathbf{C}^{[r]}$ the polynomial ring in $r$ variables, consider the surjective homomorphisms

$$
\varphi: \mathbf{C}^{[r]}:=\mathbf{C}\left[x_{1}, \ldots, x_{r}\right] \longrightarrow A, x_{i} \longmapsto a_{i}, i=1, \ldots, r,
$$

resp.

$$
\begin{gathered}
\varphi_{I}: \mathbf{C}^{[r+s+1]}=\mathbf{C}\left[x_{1}, \ldots, x_{r}, y_{0}, \ldots, y_{s}\right] \rightarrow A[I t], \\
x_{i} \longmapsto a_{i}, y_{j} \longmapsto b_{j} t, i=1, \ldots, r, j=0, \ldots, s .
\end{gathered}
$$

Let $X$ resp. $Y$ be the image of the associated closed embedding $\hat{\varphi}: \operatorname{spec} A \hookrightarrow \mathbf{C}^{r} \operatorname{resp}_{\dot{\mathrm{H}}}$ $\widehat{\varphi}_{I}: \operatorname{spec} A[I t] \hookrightarrow \mathbf{C}^{r+s+1}$. Then $X$ and $Y$ are reduced irreducible affine varieties $\mathbf{B}$ (indeed, $A[I t] \subset A[t]$ is an affine domain / C ). The principal ideal $(1-f t) \subset A[I t]$ being prime $A$, the $\mathbf{C}$-algebra $A^{\prime}$ is an affine domain / $\mathbf{C}$, too. Thus, spec $A^{\prime}$ is a reduced irreducible affine variety isomorphic to the hypersurface $V(1-f t) \subset \operatorname{spec} A[I t] \simeq Y$.

Denote by $\rho: A[I t] \longrightarrow A^{\prime}=A[I t] /(1-f t)$ the canonical surjection. Since $\varphi_{I}(1-f t)=1-y_{0}$, the image $X^{\prime}$ of the closed embedding $\widehat{\varphi_{I} \circ \rho}: \operatorname{spec} A^{\prime} \hookrightarrow \mathbf{C}^{r+s+1}$ coincides with the hyperplane section $X^{\prime}=H^{\prime} \cdot Y \simeq V(1-f t)$ of $Y$ defined by the affine hyperplane $H^{\prime}:=\left\{y_{0}=1\right\} \simeq \mathbf{C}^{r+s}$.

Definition 1.2. We call $X^{\prime} \subset \mathbf{C}^{r+s}$ as above the affine modification of the affine variety $X \subset \mathbf{C}^{r}$ with the locus $(I, f)$ (or in other words, along the divisor $D_{f}$ with center $I$ ); we denote $X^{\prime}=\Sigma_{I, f}(X)$.

Thus, this definition takes into account the distinguished systems of generators $a_{1}, \ldots, a_{r}$ of the algebra $A$ resp. $b_{0}=f, b_{1}, \ldots, b_{s}$ of the ideal $I$, that is, the closed embeddings spec $A \simeq X \hookrightarrow \mathbf{C}^{r}$ and spec $A^{\prime} \simeq X^{\prime} \hookrightarrow \mathbf{C}^{r+s}$.

\section{Remarks}

1.1. For a Noetherian triple $(A, I, f)$ and a fixed system of generators $b_{0}=f, b_{1}, \ldots, b_{s}$ of the ideal $I$ one may consider the closed embedding $\widehat{\beta}: \operatorname{spec} A^{\prime} \hookrightarrow \operatorname{spec} A \times \mathbf{C}^{s}$ associated with the surjective homomorphism of the polynomial algebra $A^{[s]}:=A\left[y_{1}, \ldots, y_{s}\right]$ over $A$ :

$$
\beta: A^{[s]} \longrightarrow A^{\prime}, \beta\left(y_{i}\right)=\rho\left(b_{i} t\right), i=1, \ldots, s .
$$

For the affine triple $(A, I, f)$ the embedding $\widehat{\varphi}_{I}:$ spec $A^{\prime} \hookrightarrow \mathbf{C}^{r+s}$ as above is the composition $\operatorname{spec} A^{\prime} \stackrel{\widehat{\beta}}{\hookrightarrow} \operatorname{spec} A \times \mathbf{C}^{s} \stackrel{\widehat{\varphi} \times \text { id }}{\hookrightarrow} \mathbf{C}^{r} \times \mathbf{C}^{s}$.

\footnotetext{
${ }^{2}$ By this reason, we do not consider here possible generalizations, for instance, such as replacing the filtration of $A$ by the powers $\left\{I^{n}\right\}_{n \in \mathbf{N}}$ of the ideal $I$ (resp. the multiplicative system $\left\{f^{n}\right\}_{n \in \mathbf{N}} \subset I$ ) by a more general one.

${ }^{3}$ We do not suppose the divisor $D_{f}$ resp. the affine subscheme spec $A / I$ in $X$ being reduced or irreducible; that is, the ideals $(f)$ and $I$ are not assumed being radical or primary.

${ }^{4}$ Indeed, the principal ideal generated by the regular function $1-f t$ in the algebra $A[t]=\mathbf{C}[X \times \mathbf{C}]$ is prime. That is, $p(t) q(t)=(1-f t) r(t)$ where $p, q, r \in A[t]$, implies that $(1-f t)$ divides $p(t)$ or $(1-f t)$ divides $q(t)$ in $A[t]$. But if, say, $(1-f t)$ divides $p(t)$ in $A[t]$, and $p \in A[I t]$, then, as it is easily seen, $(1-f t)$ divides $p(t)$ in $A[I t]$.
} 
1.2. Denote $Y^{*}=Y \backslash V_{Y}$ where $Y \subset X \times \mathbf{C}^{s+1} \subset \mathbf{C}^{r+s+1}$ is the affine cone introduced above with the vertex set $V_{Y}:=X \times\{\overline{0}\}$. Then the blow up

$$
Z=B l_{I} X=\operatorname{Proj}_{A} B l_{I} A=\operatorname{Proj}_{A} A[I t] \subset X \times \mathbf{P}^{s} \subset \mathbf{C}^{r} \times \mathbf{P}^{s}
$$

of $X$ with center $I$ is the canonical projection of $Y^{*}$, that is, $Z=Y^{*} / \mathbf{C}^{*}$ [Ei, $\left.\S 5.2\right]$. This projection restricted to the hyperplane section $X^{\prime}=H^{\prime} \cap Y$ yields an isomorphism $X^{\prime} \stackrel{\simeq}{\longrightarrow} Z \backslash H_{0}$ where $H_{0} \subset \mathbf{P}^{s}$ is the coordinate hyperplane $y_{0}=0$.

We denote by $E$ the exceptional divisor $\sigma_{I}^{-1}(C) \subset Z$ where $C:=V(I) \subset X$ and $\sigma_{I}: Z \rightarrow X$ is the blowup morphism, and by $E^{\prime}$ its affine part $E^{\prime}=E \backslash H_{0} \subset X^{\prime}$.

1.3. In the sequel we distinguish between two notions of proper transform of the divisor $D_{f}$; for that we use two terms, proper transform and strict transform, respectively. Namely, by the proper transform $D_{f}^{p r}$ of $D_{f}$ in $Z$ we mean the set of homogeneous prime ideals $p \in Z=\operatorname{Proj}_{A} A[I t]$ such that $f t \in p$. Thus, supp $D_{f}^{p r}=\left\{y_{0}=f t=0\right\}=$ $H_{0} \cap Z$, and so, $X^{\prime} \simeq Z \backslash H_{0}=Z \backslash D_{f}^{p r}$. Whereas the strict transform $D_{f}^{\prime}$ of $D_{f}$ in $Z$ we understand as usually, i.e. as the closure in $Z$ of the preimage $\sigma_{I}^{-1}(D \backslash V(I))$. In general, these two transforms are different; see Examples 1.1 and 3.4 below.

1.4. In the case when $D:=D_{f}$ is a reduced effective divisor and $I$ is the (radical) ideal of a closed reduced subvariety $C=V(I) \subset \operatorname{reg} D$ in $X$, the affine modification $X^{\prime}=\Sigma_{I, f}(X)=: \Sigma_{C, D}(X)$ coincides with the one considered in [Ka 1].

Notation. Denote Frac $A$ the field of fractions of a domain $A$. For an affine triple $(A, I, f), A[I / f]$ denotes the subalgebra of the field Frac $A$ generated over $A$ by the elements $g / f$ where $g$ runs over the ideal $I$. Under the above conventions we have

$$
A[I / f]=A\left[b_{1} / f, \ldots, b_{s} / f\right]=\left\{a / f^{k} \in \operatorname{Frac} A \mid a \in I^{k}, k=0,1, \ldots\right\} .
$$

For an affine variety $X$ we denote by $\mathbf{C}[X]$ the algebra of regular functions on $X$, and by $\mathbf{C}(X)$ the rational function field Frac $\mathbf{C}[X]$ of $X$.

Proposition 1.1: The first properties of the affine modification.

(a) In the notation as above, the affine modification $X^{\prime}=\Sigma_{I, f}(X)$ is isomorphic to the complement $Z \backslash D_{f}^{p r}$.

(b) There is a canonical isomorphism $\alpha: A^{\prime} \stackrel{\simeq}{\longrightarrow} A[I / f]=A\left[b_{1} / f, \ldots, b_{s} / f\right]$ which sends $\rho(A) \simeq A$ isomorphically onto $A$ and $\rho\left(b_{i} t\right)$ into $b_{i} / f, i=1, \ldots, s$. Thus, $X^{\prime}=\operatorname{spec} A^{\prime} \simeq \operatorname{spec} A[I / f]$.

(c) (E.D. Davis Da]) Consider the surjective homomorphism

$$
\beta: A^{[s]}=A\left[y_{1}, \ldots, y_{s}\right] \longrightarrow A[I / f]=A\left[b_{1} / f, \ldots, b_{s} / f\right] \simeq A^{\prime}
$$

where $\beta\left(y_{i}\right)=b_{i} / f, i=1, \ldots, s$. Denote by $I^{\prime}$ the ideal of the polynomial algebra $A^{[s]}$ generated by the elements $L_{1}, \ldots, L_{s} \in \operatorname{ker} \beta$ where $L_{i}=f y_{i}-b_{i}$. Then $\operatorname{ker} \beta=I^{\prime}$ (that $i s$, the subvariety $X^{\prime} \subset X \times \mathbf{C}^{s}$ is defined by the equations $\left.f y_{i}-b_{i}=0, i=1, \ldots, s\right)$ iff $I^{\prime}$ is a prime ideal, i.e. spec $A^{[s]} / I^{\prime}$ is a reduced irreducible subvariety in $X \times \mathbf{C}^{s}$. The latter is true, for instance, if the system of generators $b_{0}=f, b_{1}, \ldots, b_{s}$ of the ideal $I$ is regulan.

\footnotetext{
${ }^{5}$ By abuse of language, usually by the exceptional divisor we mean its support.

${ }^{6}$ By abuse of notation, we write $Z \backslash D_{f}^{p r}$ instead of $Z \backslash \operatorname{supp} D_{f}^{p r}$.

${ }^{7}$ I.e. for each $i=1, \ldots, s$ the image of $b_{i}$ is not a zero divisor in $A /\left(b_{0}, \ldots, b_{i-1}\right)$.
} 
(d) Furthermore, if $\operatorname{ker} \beta=I^{\prime}$ then we have an isomorphism $E^{\prime} \simeq C \times \mathbf{C}^{s}$ where $E^{\prime} \subset X^{\prime}$ is the exceptional divisor and $C=V(I)$.

Proof. For the proof of (a) see Remarks 1.2 and 1.3 above, and for that of (c) see Da, Prop. 2], [Fu], (A.6.1)] or also [Mik]. The statement (d) immediately follows from (c). Indeed, by (c), we have

$$
E^{\prime}=\sigma_{I}^{-1}(C)=\left\{(x, \bar{y}) \in X \times \mathbf{C}^{s} \mid b_{i}(x)=0, i=0, \ldots, s\right\}=C \times \mathbf{C}^{s} \subset X \times \mathbf{C}^{s} .
$$

(b) On $X^{\prime}=H^{\prime} \cap Y$ we have $t=1 / f$, and $y_{i}=b_{i} t, i=1, \ldots, s$. Thus, $A^{\prime}=\mathbf{C}\left[X^{\prime}\right]=$ $A\left[1, y_{1}, \ldots, y_{s}\right] \mid X^{\prime}=A\left[b_{1} / f, \ldots, b_{s} / f\right]$, as stated.

It is well known (see e.g. [Ha, 7.17]) that every birational projective morphism of quasiprojective varieties $Y \rightarrow X$ is a blow up of $X$ with center at a subsheaf of ideals $\mathcal{I} \subset \mathcal{O}_{X}$. Similarly, we have the following theorem.

\section{Theorem 1.1: Birational morphisms as affine modifications.}

Any birational morphism $q: Y \rightarrow X$ of affine varieties is an affine modification. More precisely, there are an ideal $I \subset A=\mathbf{C}[X]$, an element $f \in I$ and an isomorphism $\alpha: Y \stackrel{\simeq}{\longrightarrow} X^{\prime}:=\Sigma_{I, f}(X)$ such that $q=\sigma_{I} \circ \alpha$ where $\sigma_{I}: X^{\prime} \rightarrow X$ is the blowup morphism with center $I$.

Proof. Denote $A_{1}=\left(q^{*}\right)^{-1}(\mathbf{C}[Y]) \subset \mathbf{C}(X)=$ Frac $A$, where $\left(q^{*}\right)^{-1}: \mathbf{C}(Y) \rightarrow \mathbf{C}(X)$ is the induced isomorphism of the rational function fields. Each function $a \in A=\mathbf{C}[X]$ being lifted by $q^{*}$ to a function in $\mathbf{C}[Y]$ comes back under the inverse homomorphism $\left(q^{*}\right)^{-1}$. Therefore, $A \subset A_{1} \subset$ Frac $A$. It is enough to show that $A_{1}=A[I / f]$ for some ideal $I$ of $A$ and some $f \in I$.

The affine domain $A_{1}$ being finitely generated we have

$$
A_{1}=A\left[a_{1} / f_{1}, \ldots, a_{k} / f_{k}\right]=A\left[b_{1} / f, \ldots, b_{k} / f\right]=A[I / f]
$$

for some $a_{i}, f_{i} \in A$ where $f=f_{1} \cdot \ldots \cdot f_{k} \in A, b_{i}=f a_{i} / f_{i} \in A$ and $I=\left(f, b_{1}, \ldots, b_{k}\right) \subset$ $A$, as desired.

Proposition 1.2: A decomposition of an affine modification. Let $f=f_{1} f_{2} \in$ I. Denote $I_{1}=\left(I, f_{1}\right)$ and $A_{1}=\Sigma_{I_{1}, f_{1}}(A)$. Consider the ideal $I_{2}$ of the algebra $A\left[I_{1} / f_{1}\right] \simeq A_{1}$ generated by the subspace $I / f_{1}$. Then we have $A^{\prime}:=\Sigma_{I, f}(A) \simeq A_{1}^{\prime}:=$ $\Sigma_{I_{2}, f_{2}}\left(A_{1}\right)$.

Respectively, denoting $X=$ spec $A, X^{\prime}=$ spec $A^{\prime}$ and $X_{1}=$ spec $A_{1}$ we obtain the decomposition $\sigma_{I}=\sigma_{I_{2}} \circ \sigma_{I_{1}}$ where $\sigma_{I}: X^{\prime} \rightarrow X, \sigma_{I_{1}}: X_{1} \rightarrow X$ and $\sigma_{I_{2}}: X^{\prime} \rightarrow X_{1}$ are the corresponding blowup morphisms.

Proof. Let $I=\left(f, b_{1}, \ldots, b_{s}\right)$. By Proposition 1.1(b), we have:

$$
\begin{gathered}
A^{\prime} \simeq A[I / f]=A\left[b_{1} / f, \ldots, b_{s} / f\right]=A\left[b_{1} / f_{1} f_{2}, \ldots, b_{s} / f_{1} f_{2}\right] \subset \operatorname{Frac} A \\
A_{1} \simeq A\left[I_{1} / f_{1}\right]=A\left[b_{1} / f_{1}, \ldots, b_{s} / f_{1}\right] \subset A[I / f] \subset \operatorname{Frac} A
\end{gathered}
$$

and $I_{1}=\left(f_{2}, b_{1} / f_{1}, \ldots, b_{s} / f_{1}\right) \subset A_{1}$. Hence,

$$
A_{1}^{\prime} \simeq A_{1}\left[I_{2} / f_{2}\right]=A_{1}\left[b_{1} / f_{1} f_{2}, \ldots, b_{s} / f_{1} f_{2}\right] \subset \operatorname{Frac} A
$$

Clearly, the latter subalgebra coincides with $A[I / f] \simeq A^{\prime}$, as claimed. 
Next we give several examples of affine modifications.

Example 1.1: Deleting a divisor. If $I=A$, that is, the height ht $I=0$, then we have $A^{\prime} \simeq A[1 / f], Z \simeq X$ and $X^{\prime} \simeq X \backslash D_{f} \simeq Z \backslash D_{f}^{p r}$.

If $I=(f)$ is a principal ideal (and so, ht $I=1$ ) we obtain $A[I / f]=A$, whence, $X^{\prime}=X \simeq Z=X \times \mathbf{P}^{0}$ whereas $\operatorname{supp} D_{f}^{p r}=\emptyset$. Thus, the equality $X^{\prime}=Z \backslash D_{f}^{p r}$ still holds.

Furthermore, even if ht $I \geq 2$ it may happen that supp $D_{f}^{p r}=\sigma_{I}^{-1}\left(\operatorname{supp} D_{f}\right)$ and so, $X^{\prime} \simeq X \backslash D_{f}$, i.e. that the hyperplane section $H_{0} \cap Z$ contains the exceptional divisor $E$ of the blow up $\sigma_{I}$. For instance, take $A=\mathbf{C}^{[2]}=\mathbf{C}[x, y]$, that is, $X=\mathbf{C}^{2}, I=(x, y)$ and $f=x^{2} \in I$. Then we have $Z=\left\{((x, y),(u: v)) \in \mathbf{C}^{2} \times \mathbf{P}^{1} \mid x v=y u\right\}$, and the curve supp $D_{f}^{p r}$ given in $Z$ by the equation $u x=0$ consists of two lines, the first one $l^{\prime}:=\{u=0\}$ being the strict transform in $Z$ of the affine line $l:=\operatorname{supp} D_{f}=\{x=0\}$ and the second one $\{x=y=0\}$ being the exceptional divisor $E \subset Z$. Thus, $X^{\prime}=$ $Z \backslash\left(l^{\prime} \cup E\right) \simeq X \backslash l \simeq \mathbf{C}^{*} \times \mathbf{C} \subset \mathbf{C}^{2} \simeq X$. While for the affine triple $(A, I, x)$ we obtain $X^{\prime}=\Sigma_{I, x}(X) \simeq \mathbf{C}^{2}$.

Example 1.2: A singular modification of the affine plane. Set $A=\mathbf{C}[x, y]$ (that is, $\left.X=\mathbf{C}^{2}\right), f=x, I=\left(x, y^{2}\right)$. The system of generators $b_{0}:=x, b_{1}=y^{2}$ of the ideal $I$ is regular. Hence, by Proposition 1.1(c), $X^{\prime}$ is the affine surface $x z=y^{2}$ in $\mathbf{C}^{3}$. It is isomorphic to the quotient of the affine plane $\mathbf{C}^{2}$ by the involution $(x, z) \longmapsto(-x,-z)$.

Example 1.3: Modification of an affine space along a hyperplane with center at a point. Let $A=\mathbf{C}\left[x_{1}, \ldots, x_{n}, y\right], f=y$, and let $I=\left(x_{1}, \ldots, x_{n}, y\right)$ be the maximal ideal corresponding to the origin of $X=\mathbf{C}^{n+1}$. Then $A^{\prime} \simeq \mathbf{C}\left[x_{1} y^{-1}, \ldots, x_{n} y^{-1}, y\right] \subset$ $\mathbf{C}\left(x_{1}, \ldots, x_{n}, y\right)$. By Proposition 1.1(c), $X^{\prime} \simeq \mathbf{C}^{n+1}$ is given in $\mathbf{C}^{2 n+1}$ by the equations $x_{i}=y y_{i}, \quad i=1, \ldots, n$, and the exceptional divisor $E^{\prime}=E \backslash D_{f}^{p r} \simeq \mathbf{C}^{n}$ is given in $X^{\prime}$ as $y=0$. In the coordinates $\left(y_{1}, \ldots, y_{n}, y\right)$ of $X^{\prime} \simeq \mathbf{C}^{n+1}$ the blowup morphism $\sigma_{I}: X^{\prime} \rightarrow X$ is given as $\sigma_{I}\left(y_{1}, \ldots, y_{n}, y\right)=\left(y y_{1}, \ldots, y y_{n}, y\right)$.

A modification of $\mathbf{C}^{n+1}$ along a coordinate hyperplane $H=D_{y} \simeq \mathbf{C}^{n}$ with center at a coordinate subspace $C=\mathbf{C}^{k} \subset \mathbf{C}^{n}$ can be described in a similar way.

Example 1.4: Modification of an affine space along a divisor with center at a codimension two complete intersection. Set $A=\mathbf{C}^{[r]}$, that is, $X=\mathbf{C}^{r}$, and $I=(f, g)$, where $f, g \in A$ are non-constant polynomials without common factor. Then $f, g$ form a regular system of generators of the ideal I. Thus, by Proposition 1.1(c), the affine modification $X^{\prime}=\Sigma_{I, f}(X)$ is the hypersurface in $\mathbf{C}^{r+1}$ with the equation $f(\bar{x}) y-g(\bar{x})=0$ where $\bar{x}=\left(x_{1}, \ldots, x_{r}\right)$. The blowup morphism $\sigma_{I}: X^{\prime} \rightarrow X$ is the restriction to $X$ of the projection $\mathbf{C}^{r+1} \rightarrow \mathbf{C}^{r},(\bar{x}, y) \rightarrow \bar{x}$. The exceptional divisor $E^{\prime} \subset X^{\prime}$ is given in $\mathbf{C}^{r+1}$ by the equations $f(\bar{x})=g(\bar{x})=0$; so, $E^{\prime} \simeq V(I) \times \mathbf{C}$.

Example 1.5: The Russell cubic threefold. In particular, set $A=\mathbf{C}[x, z, t]$ (that is, $\left.X=\mathbf{C}^{3}\right), \quad f=-x^{2}$ and $I=(f, g)$ where $g=x+z^{2}+t^{3}$. Then the affine modification $X^{\prime}=\Sigma_{I, f}(X)$ is the smooth 3-fold $x+x^{2} y+z^{2}+t^{3}=0$ in $\mathbf{C}^{4}$. We call it the Russell cubic (see [Ru 1]). It birationally dominates the affine space $\mathrm{C}^{3}$ via the blowup morphism $\sigma_{I}: X^{\prime} \rightarrow X \simeq \mathbf{C}^{3}, \sigma_{I}:(x, y, z, t) \longmapsto(x, z, t)$.

In turn, the Russell cubic threefold $X^{\prime} \subset \mathbf{C}^{4}$ is birationally dominated 1 by $\mathbf{C}^{3}$. Indeed, for any $x \neq 0, y$ is expressed in terms of $z$ and $t$; whence, the part $\{x \neq 0\}$

${ }^{8}$ This observation is due to P. Russell. 
of the threefold $X^{\prime}$ is isomorphic to $\mathbf{C}^{2} \times \mathbf{C}^{*}$. The 'book-surface' $B:=\{x=0\} \subset X^{\prime}$ is the product $\mathbf{C} \times \Gamma_{2,3}$ where $\Gamma_{2,3}:=\left\{z^{2}+t^{3}=0\right\} \subset \mathbf{C}^{2}$. Fix a smooth point $\rho \in \Gamma_{2,3}$, and perform the affine modification $\sigma^{\prime}: X^{\prime \prime} \longrightarrow X^{\prime}$ of $X^{\prime}$ along $B$ with the center $C:=\mathbf{C} \times\{\rho\}$. In this way we replace $B$ by a smooth surface $E^{\prime} \simeq \mathbf{C}^{2}$ and replace the function $x$ by a function $h: X^{\prime \prime} \longrightarrow \mathbf{C}$ such that all the fibers of $h$ are smooth reduced surfaces isomorphic to $\mathbf{C}^{2}$. Using an explicit presentation of $X^{\prime \prime}$ it can be checked that $X^{\prime \prime} \simeq \mathbf{C}^{3}$ (see Example 6.3 below), and so, $\sigma^{\prime}: \mathbf{C}^{3} \simeq X^{\prime \prime} \longrightarrow X^{\prime}$ is a birational (whence, dominant) morphism.

It is known that the Russell cubic is diffeomorphic to $\mathbf{C}^{3}$ (see e.g. $\mathbb{R u} 1$, Ka 1, Za 2 or Example 3.2 below). However, by a theorem of Makar-Limanov [ML 1] (or also $\mathrm{DA}$, KaML 1, Za 2]), it is not isomorphic to $\mathbf{C}^{3}$. A smooth affine variety which is diffeomorphic but non-isomorphic to $\mathbf{C}^{n}$ is called an exotic $\mathbf{C}^{n}$. Thus, the Russell cubic $X^{\prime}$ is an exotic $\mathbf{C}^{3}$ of sandwich type, that is, there are birational morphisms $\mathrm{C}^{3} \rightarrow X^{\prime} \rightarrow \mathrm{C}^{3}$.

Example 1.6: Decomposing an affine modification. Let $A=\mathbf{C}^{[2]}=\mathbf{C}[x, y], I=$ $(f, g)$ where $f, g \in \mathbf{C}[x, y]$ and $f=f_{1} f_{2}$. Set $A_{1}=\mathbf{C}^{[3]} /\left(f_{1} z-g\right)$ (see Example 1.4), and let $\sigma_{I}$ be the restriction of $\sigma_{I_{2}} \circ \sigma_{I_{1}}$ to the surface $f z-g=0$ where $\sigma_{I_{1}}: \mathbf{C}^{3} \rightarrow$ $\mathbf{C}^{2}, \sigma_{I_{1}}:(x, y, z) \longmapsto(x, y)$, and $\sigma_{I_{2}}: \mathbf{C}^{3} \rightarrow \mathbf{C}^{3},(x, y, z) \longmapsto\left(x, y, f_{2}(x, y) z\right)$ is the affine modification of $\mathbf{C}^{3}$ along the divisor $D_{f_{2}} \times \mathbf{C}$ with center $D_{f_{2}} \simeq \operatorname{spec} \mathbf{C}^{[3]} /\left(f_{2}, z\right)$.

\section{The universal property of affine modifications}

Here we show that the universal property of the blow ups (see e.g. Hird) is still valid for the affine modifications.

Proposition 2.1: Lifting a homomorphism to affine modifications. Consider two affine triples $(A, I, f)$ and $\left(A_{1}, I_{1}, f_{1}\right)$ and their affine modifications $A^{\prime}=\Sigma_{I, f}(A) \simeq$ $A[I / f]$ resp. $A_{1}^{\prime}=\Sigma_{I_{1}, f_{1}}\left(A_{1}\right) \simeq A_{1}\left[I_{1} / f_{1}\right]$. Let $\mu: A \rightarrow A_{1}$ be a homomorphism such that $\mu(I) \subset I_{1}$ and $\mu(f)=\alpha f_{1}$ where $\alpha \in A_{1}$ is an invertible element. Then there exists a unique homomorphism $\mu^{\prime}: A^{\prime} \rightarrow A_{1}^{\prime}$ which extends $\mu$; it can be defined as follows:

$$
\mu^{\prime}: A[I / f] \ni a / f^{k} \longmapsto \alpha^{-k} \mu(a) / f_{1}^{k} \in A_{1}\left[I_{1} / f_{1}\right] .
$$

If, in addition, $\mu(A)=A_{1}$ and $\mu(I)=I_{1}$, then $\mu^{\prime}\left(A^{\prime}\right)=A_{1}^{\prime}$.

Proof. By Proposition 1.1(b) (see also Remark 1.3 above), there are canonical isomorphisms $A^{\prime} \simeq A[I / f]$ resp. $A_{1}^{\prime} \simeq A_{1}\left[I_{1} / f_{1}\right]$. Thus, it is enough to show that $\mu$ admits a unique extension $\mu^{\prime}: A[I / f] \rightarrow A_{1}\left[I_{1} / f_{1}\right]$, which is surjective if so are $\mu$ and $\mu \mid I: I \rightarrow I_{1}$. Furthermore, since $A_{1}\left[I_{1} / f_{1}\right]=A_{1}\left[I_{1} / \alpha f_{1}\right]$, replacing $\alpha f_{1}$ by $f_{1}$ we may suppose in the sequel that $\alpha=1$, i.e. that $f_{1}=\mu(f)$.

Consider the natural extension $\tilde{\mu}: A[t] \rightarrow A_{1}[\tau], \tilde{\mu} \mid A=\mu, \tilde{\mu}(t)=\tau$. Since $\tilde{\mu}$ sends the principal ideal $(1-f t) \subset A$ into the principal ideal $\left(1-f_{1} \tau\right) \subset A_{1}$, it induces a homomorphism $\widehat{\mu}: A[t] /(1-f t) \rightarrow A_{1}[\tau] /\left(1-f_{1} \tau\right)$. In this way, via the canonical isomorphisms $A[t] /(1-f t) \simeq A[1 / f] \subset$ Frac $A$ resp. $\quad A_{1}[\tau] /\left(1-f_{1} \tau\right) \simeq A_{1}\left[1 / f_{1}\right] \subset$ Frac $A_{1}$, we obtain an extension $\widehat{\mu}: A[1 / f] \rightarrow A_{1}\left[1 / f_{1}\right]$ of $\mu$. Any such extension sends a generic element $a / f^{k} \in A[1 / f]$ into the element $\mu(a) / \mu\left(f^{k}\right)=\mu(a) / f_{1}^{k} \in A_{1}\left[1 / f_{1}\right]$. Therefore, $\widehat{\mu}$ is uniquely defined on $A[1 / f]$ by the formula $\widehat{\mu}\left(a / f^{k}\right)=\mu(a) / f_{1}^{k}$. 
Observe that an element $a / f^{k} \in A[1 / f]$ belongs to $A[I / f]$ iff $a \in I^{k}$. Since by assumption, $\mu\left(I^{k}\right) \subset I_{1}^{k}, \quad k=0,1, \ldots$, we have $\widehat{\mu}(A[I / f]) \subset A_{1}\left[I_{1} / f_{1}\right]$. So, $\mu^{\prime}:=$ $\widehat{\mu} \mid A[I / f]$ is a desired extension, and, clearly, it is unique.

Now, if $\mu(A)=A_{1}$ and $\mu(I)=I_{1}$, then $\mu\left(I^{k}\right)=I_{1}^{k}, k=0,1, \ldots$ Hence, any element $a_{1} / f_{1}^{k} \in A_{1}\left[I_{1} / f_{1}\right]$ can be written as $\mu^{\prime}\left(a / f^{k}\right)$ with $a \in I^{k}$. Therefore, in this case $\mu^{\prime}$ is a surjection.

Definition 2.1. Let $J \subset A$ be a prime ideal such that $f \notin J$. We define the strict transform $J^{\text {st }}$ of $J$ in $A^{\prime}=A[I / f]$ as follows:

$$
J^{\text {st }}=\left\{a^{\prime} \in A^{\prime} \mid f^{k} a^{\prime} \in J \text { for some } k \in \mathbf{N}\right\} .
$$

It is easily seen that $J^{\text {st }} \subset A^{\prime}$ is a prime ideal containing $J$.

The following statements complete Proposition 2.1.

\section{Proposition 2.2: How does a modification affect a subvariety.}

(a) Let $(A, I, f)$ be an affine triple, and let $J$ be a prime ideal in $A$ such that $f \notin J$. Denote by $\mu: A \longrightarrow A_{1}:=A / J$ the canonical surjection. Set $I_{1}=\mu(I), f_{1}=\mu(f)$, and $A_{1}^{\prime}=\Sigma_{I_{1}, f_{1}}\left(A_{1}\right)$. Then $A^{\prime} / J^{\text {st }} \simeq A_{1}^{\prime}$.

(b) Furthermore, set $X=\operatorname{spec} A$, and let $X_{1}$ be an irreducible closed subvariety of $X$ which is not contained in the support of $D_{f}$. Denote by $J=I\left(X_{1}\right)$ the defining ideal of $X_{1}=$ spec $A_{1}$, and set $X^{\prime}=\Sigma_{I, f}(X), X_{1}^{\prime}=\Sigma_{I_{1}, f_{1}}\left(X_{1}\right)$ where $I_{1}, f_{1}$ are as in (a). Then the strict transform $J^{\text {st }}$ of the ideal $J$ in $A^{\prime}=\mathbf{C}\left[X^{\prime}\right]$ coincides with the defining ideal of the strict transform $X_{1}^{\text {st }}$ of $X_{1}$ in $X^{\prime}$, and the variety $X_{1}^{\text {st }}$ is isomorphic to $X_{1}^{\prime}$.

Proof. (a) Let $\mu^{\prime}: A^{\prime} \longrightarrow A_{1}^{\prime}$ be a surjective extension of $\mu$ as in Proposition 2.1. We have to show that $\operatorname{ker} \mu^{\prime}=J^{\text {st }}$. Note that $J=\operatorname{ker} \mu \subset \operatorname{ker} \mu^{\prime}$, and $f \notin \operatorname{ker} \mu^{\prime}$, since $\mu^{\prime}(f)=\mu(f) \neq 0$ in $A_{1} \subset A_{1}^{\prime}$. For an element $a^{\prime} \in \operatorname{ker} \mu^{\prime}$ chose $k \in \mathbf{N}$ so that $f^{k} a^{\prime} \in A$. Then $0=\mu^{\prime}\left(f^{k} a^{\prime}\right)=\mu\left(f^{k} a^{\prime}\right)$, that is, $f^{k} a^{\prime} \in J$, and hence, $a^{\prime} \in J^{\text {st }}$. Thus, ker $\mu^{\prime} \subset J^{\text {st }}$.

Vice versa, let $a^{\prime} \in J^{\text {st }}$, and let $k \in \mathbf{N}$ be such that $f^{k} a^{\prime} \in J=\operatorname{ker} \mu$. Thus, $\mu^{\prime}\left(f^{k} a^{\prime}\right)=\mu\left(f^{k} a^{\prime}\right)=0$. But $\mu\left(f^{k}\right) \neq 0$. Since $A_{1}$ and $A_{1}^{\prime}$ are domains, the equality $\mu^{\prime}\left(f^{k}\right) \mu^{\prime}\left(a^{\prime}\right)=0$ implies $\mu^{\prime}\left(a^{\prime}\right)=0$, i.e. $a^{\prime} \in \operatorname{ker} \mu^{\prime}$. Therefore, $J^{\text {st }} \subset \operatorname{ker} \mu^{\prime}$, and hence, $J^{\text {st }}=\operatorname{ker} \mu^{\prime}$. This proves (a).

(b) Observe that $g^{\prime} \in A^{\prime}=\mathbf{C}\left[X^{\prime}\right]$ vanishes on the strict transform $X_{1}^{\text {st }}$ of $X_{1}$ iff so does $f^{k} g^{\prime}$. If $k$ is large enough, then we have $f^{k} g^{\prime} \in A=\mathbf{C}[X] \hookrightarrow \mathbf{C}\left[X^{\prime}\right]$, and $f^{k} g^{\prime} \mid X_{1}^{\text {st }}=0$ implies that $f^{k} g^{\prime} \mid X_{1}=0$. Thus, $g^{\prime} \in I\left(X_{1}^{\text {st }}\right)$ iff $f^{k} g^{\prime} \in I\left(X_{1}\right)=J$ for large enough $k$, i.e. iff $g^{\prime} \in J^{\text {st }}$. Hence, $I\left(X_{1}^{\text {st }}\right) \subset J^{\text {st }}$.

Conversely, let $g^{\prime} \in J^{\text {st }}$, i.e. $f^{k} g^{\prime} \in J=I\left(X_{1}\right)$ for large enough $k$. Thus, $f^{k} g^{\prime} \mid X_{1}=$ 0 , which implies that $g^{\prime} \mid X_{1}^{\text {st }}=0$. Therefore, $J^{\text {st }} \subset I\left(X_{1}^{\text {st }}\right)$, or $J^{\text {st }}=I\left(X_{1}^{\text {st }}\right)$. Now (a) provides an isomorphism $X_{1}^{\text {st }}=\operatorname{spec} A^{\prime} / J^{\text {st }} \simeq \operatorname{spec} A_{1}^{\prime}=X_{1}^{\prime}$. The proof is completed.

Corollary 2.1: Restricting affine modification to a subvariety. Let $X=$ spec $A$ be an affine variety, and $X_{1}=$ spec $A_{1}$ be an irreducible closed subvariety of $X$. Fix a proper ideal $I \subset A$, and let the ideal $I_{1} \subset A_{1}$ consists of the restrictions to $X_{1}$ of the elements of $I$. Fix also an element $f \in I$ such that $f_{1}:=f \mid X_{1} \neq 0$. Then there is a unique closed embedding $i^{\prime}: X_{1}^{\prime} \hookrightarrow X^{\prime}$ making the following diagram commutative: 


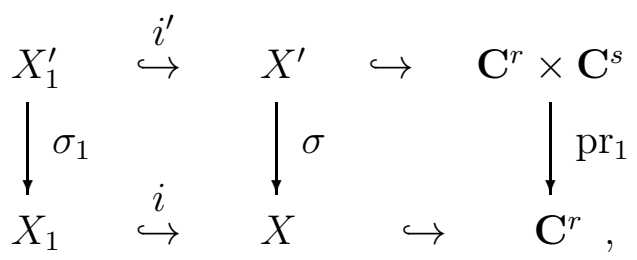

where $\sigma: X^{\prime} \rightarrow X$ resp. $\quad \sigma_{1}: X_{1}^{\prime} \rightarrow X_{1}$ is the blowup morphism of the affine modification $X^{\prime}=\Sigma_{I, f}(X)$ resp. $\quad X_{1}^{\prime}=\Sigma_{I_{1}, f_{1}}\left(X_{1}\right), \quad i: X_{1} \hookrightarrow X$ is the identical embedding, and $p r_{1}$ is the first projection.

Remark 2.1. In particular, affine modifications commute with direct products.

Example 2.1: Modification of an affine hypersurface along a hyperplane section with center at a point. Let $g \in \mathbf{C}\left[x_{1}, \ldots, x_{n}, y\right]$ be an irreducible polynomial such that $g(\overline{0})=0$. Set $X=\mathbf{C}^{n+1}$ and $X_{1}=g^{-1}(0) \subset X$. That is, $X=\operatorname{spec} A$ resp. $X_{1}=\operatorname{spec} A_{1}$ where $A=\mathbf{C}^{[n+1]} \operatorname{resp} . \quad A_{1}:=\mathbf{C}^{[n+1]} / J, J:=(g)$. Set $I=$ $\left(x_{1}, \ldots, x_{n}, y\right) \subset A, \quad I_{1}=\left(x_{1}+(g), \ldots, x_{n}+(g), y+(g)\right) \subset A_{1}, \quad f=y \in I$ and $f_{1}=y+(g) \in I_{1}$. As in Example 1.3 above consider the new coordinates $\left(y_{1}, \ldots, y_{n}, y\right)$ in $X^{\prime} \simeq \mathbf{C}^{n+1}$ where the blowup morphism $\sigma_{I}: X^{\prime} \rightarrow X$ is given by $x_{i}=y y_{i}, i=1, \ldots, n$. Represent $g\left(y y_{1}, \ldots, y y_{n}, y\right)=y^{\mu} g_{1}\left(y_{1}, \ldots, y_{n}, y\right)$ where $g_{1} \in \mathbf{C}\left[y_{1}, \ldots, y_{n}, y\right]$ is not divisible by $y$. Then the equation of the affine modification $X_{1}^{\prime}=X_{1}^{\text {st }}$ of $X_{1}$ along the divisor $D_{f_{1}}$ with center $I_{1}$ in $X^{\prime} \simeq \mathbf{C}^{n+1}=\Sigma_{I, f}(X)$ is $g_{1}=0$.

Example 2.2: Modification of an affine space along a divisor with center at a point (cf. [Za 2, Cor. 6.1]). Let $p \in \mathbf{C}^{[k]}$ be a non-constant polynomial such that $p(\overline{0})=0$. Represent $\mathbf{C}^{k}$ as the hypersurface $X$ in $\mathbf{C}^{k+1}$ with the equation $y-p(\bar{x})=0$. Then as above, the affine modification $X^{\prime}=\Sigma_{(\overline{0}), p} \mathbf{C}^{k}=\Sigma_{(\overline{0}), y} X$ is isomorphic to the hypersurface in $\mathbf{C}^{k+1}$ with the equation $\frac{p(y \bar{x})-y}{y}=0$.

Corollary 2.2: Lifting automorphisms to an affine modification. (a) Let $(A, I, f)$ be an affine triple, and let $\varphi \in$ Aut $A$ be an automorphism such that $\varphi(I)=I$ and $\varphi(f)=\alpha f$ where $\alpha \in A$ is an invertible element. Then there exists a unique extension $\varphi^{\prime} \in$ Aut $A^{\prime}$ of $\varphi$ to an automorphism of the affine modification $A^{\prime}=\Sigma_{I, f}(A)$.

(b) Furthermore, the induced automorphism $\hat{\varphi}$ of $X=$ spec $A$ preserves the divisor $D=D_{f}$ and the center $C=V(I)$ of the blow up. It can be lifted in a unique way to an automorphism $\hat{\varphi}^{\prime}$ of the affine modification $X^{\prime}=\Sigma_{I, f}(X)$ which preserves the exceptional divisor $E^{\prime} \subset X^{\prime}$ of the blow up; in the complement $X^{\prime} \backslash E^{\prime}$ we have $\hat{\varphi}^{\prime}=$ $\sigma_{I}^{-1} \circ \varphi^{\prime} \circ \sigma_{I}$ where $\sigma_{I}: X^{\prime} \rightarrow X$ is the blowup morphism.

Remark 2.2. In particular, let $(X, C, D)$ be a triple as in Remark 1.4 above, and let $\widehat{\varphi} \in$ Aut $X$ be an automorphism of $X$ such that $\hat{\varphi}(D)=D, \widehat{\varphi}(C)=C$. Then there exists a unique automorphism $\hat{\varphi}^{\prime} \in$ Aut $X^{\prime}$ of the affine modification $X^{\prime}=\Sigma_{C, D}(X)$ such that $\hat{\varphi}^{\prime}\left|\left(X^{\prime} \backslash E^{\prime}\right)=\hat{\varphi}\right|(X \backslash D)$ under the natural identification $\sigma_{C} \mid\left(X^{\prime} \backslash E^{\prime}\right)$ : $X^{\prime} \backslash E^{\prime} \stackrel{\simeq}{\longrightarrow} X \backslash D$.

Example 2.3. Let $A=\mathbf{C}^{[3]}=\mathbf{C}[x, y, z]$, that is, $X=\mathbf{C}^{3}, I=(x, z)$, and $f=x$. Then $X^{\prime} \simeq \mathbf{C}^{3}$, and $\sigma_{I}^{*}: A \rightarrow A^{\prime}=\mathbf{C}\left[x^{\prime}, y^{\prime}, z^{\prime}\right]$ is given as

$$
\sigma_{I}^{*}:(x, y, z) \longmapsto\left(x^{\prime}, y^{\prime}, x^{\prime} z^{\prime}\right)
$$


(cf. Examples 1.3, 1.4). Let $\mu \in$ Aut $A$ be given by

$$
\mu:(x, y, z) \longmapsto\left(x, y+x g_{1}(x, y, z), z+x g_{2}(x, y, z)\right)
$$

where $g_{1}, g_{2} \in \mathbf{C}^{[3]}$. Then we have $\mu(I)=I, \mu(f)=f$, and the extension $\mu^{\prime} \in$ Aut $A^{\prime}$ can be given as

$$
\mu^{\prime}:\left(x^{\prime}, y^{\prime}, z^{\prime}\right) \longmapsto\left(x^{\prime}, y^{\prime}+x^{\prime} g_{1}\left(x^{\prime}, y^{\prime}, x^{\prime} z^{\prime}\right), z^{\prime}+g_{2}\left(x^{\prime}, y^{\prime}, x^{\prime} z^{\prime}\right)\right) .
$$

Recall the following notion.

Definition 2.2. Let $A$ be a commutative algebra over $\mathbf{C}$, and let $\partial$ be a derivation of $A$. It is called locally nilpotent if for any $a \in A$ we have $\partial^{n}(a)=0$ for some $n=n(\partial, a)$. For an algebra $A$ denote by LND $(A)$ the set of all locally nilpotent derivations (LND-s for short) of $A$. Giving an LND $\partial$ on a Noetherian $\mathbf{C}$ - algebra $A$ is the same as giving a regular $\mathbf{C}_{+}-\operatorname{action} \theta \varphi_{\partial}$ on spec $A$. For $a \in A$ and $t \in \mathbf{C}_{+}$we put

$$
\varphi_{\partial}(t, a)=\exp (t \partial)(a)=\sum_{i=0}^{n(\partial, a)-1} \frac{t^{i} \partial^{i} a}{i !}
$$

[R]. The kernel ker $\partial$ coincides with the subalgebra $A^{\varphi_{\partial}} \subset A$ of $\varphi_{\partial}$-invariants.

Corollary 2.3: Lifting LND-s and $\mathrm{C}_{+}-$actions to an affine modification. Let $(A, I, f)$ be an affine triple, and set $X=$ spec $A$. Let $\partial$ be an LND of the algebra $A=\mathbf{C}[X]$ such that $\partial(f)=0$ and $\partial(I) \subset I$. Then $\partial$ can be lifted in a unique way to an LND $\partial^{\prime}$ of the affine modification $A^{\prime}=\mathbf{C}\left[X^{\prime}\right]=\Sigma_{I, f}(A)$. The corresponding $\mathbf{C}_{+}-$action $\varphi_{\partial}$ on $X$ (which leaves invariant the subvarieties $D_{f}$ and $C=V(I)$ ) can be lifted in a unique way to a $\mathbf{C}_{+}-$action $\varphi_{\partial}^{\prime}$ on the affine modification $X^{\prime}=\Sigma_{I, f}(X)$ which leaves invariant the exceptional divisor $E^{\prime}$.

Example 2.4. Let $A=\mathbf{C}[x, y]$, i.e. $X=\mathbf{C}^{2}$, and $A^{\prime}=\Sigma_{I, x}(A)$ where $I=$ $(x, q(y)), q \in \mathbf{C}[y]$. Then $X^{\prime}=\Sigma_{I, x}\left(\mathbf{C}^{2}\right)$ is the surface given in $\mathbf{C}^{3}$ by the equation $x z-q(y)=0$, and the blowup morphism $\sigma_{I}: X \rightarrow \mathbf{C}^{2}$ is the restriction to $X$ of the standard projection $\mathbf{C}^{3} \rightarrow \mathbf{C}^{2},(x, y, z) \longmapsto(x, y)$ (see Example 1.4).

Consider the triangular $\mathbf{C}_{+}$-action $\varphi(t,(x, y))=(x, y+t x p(x))$ on $\mathbf{C}^{2}$ where $p \in \mathbf{C}[x]$, and the corresponding LND $\partial: A \rightarrow A$,

$$
\partial:(x, y) \longmapsto(0, x p(x)) .
$$

Clearly, $\partial(I) \subset I$. By Corollary 2.3, there exist unique extensions $\partial^{\prime}$ resp. $\varphi^{\prime}$ of $\partial$ resp. $\varphi$ to $A^{\prime}$; they are given, respectively, as follows:

$$
\partial^{\prime}:(x, y, z=q(y) / x) \longmapsto\left(0, x p(x), q^{\prime}(y) p(x)\right)
$$

and

$$
\varphi^{\prime}:(x, y, z) \longmapsto\left(x, y+\operatorname{txp}(x), q(y+t x p(x)) / x=z+\sum_{i=0}^{\operatorname{deg} q} t^{q^{(i)}(y)} \frac{{ }^{i-1}}{i !} x^{i}(x)\right) .
$$

\footnotetext{
${ }^{9}$ Here $\mathbf{C}_{+}$stands for the additive group of the complex number field.
} 


\section{Topology of affine modifications}

In this section we appropriate the complex analytic point of view. Observe that, with the language of schemes, one can naturally extend the notion of the affine modification to quasiprojective varieties and to more general ring spaces and obtain results analogous to those of the previous sections. Instead, to simplify things, according to Proposition 1.1(a) and Theorem 1.1 we adopt the following definitions.

Definition 3.1. Consider a triple $(M, C, D)$ resp. a pair $\left(M^{\prime}, E^{\prime}\right)$ where $M$ resp. $M^{\prime}$ is a reduced connected complex space, $D \subset M$ resp. $E^{\prime} \subset M^{\prime}$ is a closed hypersurface, and $C \subset D$ is a closed analytic subvariety of codimension at least two in $M$. Let $\sigma: M^{\prime} \rightarrow M$ be a surjective morphism such that the restriction $\sigma \mid\left(M^{\prime} \backslash E^{\prime}\right): M^{\prime} \backslash E^{\prime} \rightarrow$ $M \backslash D$ is a biholomorphism, and $\sigma\left(E^{\prime}\right) \subset C$. Then we say that the pair $\left(M^{\prime}, E^{\prime}\right)$ is a pseudoaffine modification (via $\sigma$ ) of the triple $(M, C, D)$ with a locus subordinated to $(C, D)$ and with the exceptional divisor $E^{\prime}$.

In particular, we consider the pseudoaffine modification $M^{\prime}=\Sigma_{C, D}(M)$ of $M$ along $D$ with center $C$, where $M^{\prime}=\widehat{M} \backslash D^{\prime}, \widehat{M}$ is the blow up of $M$ at $C$ and $D^{\prime}$ is the strict transform of $D$ in $\widehat{M}$.

Anyhow, modifying $M$ we replace the divisor $D$ by the new one $E^{\prime}$. In the latter case $E^{\prime}=E \backslash D^{\prime}$ where $E=\sigma_{C}^{-1}(C) \subset \widehat{M}$ is the exceptional divisor of the blow up $\sigma_{C}: \widehat{M} \rightarrow M$ with center $C$. While in the former case we blow up $M$ with center being an ideal sheaf supported by $C$.

Recall that by a vanishing loop of a divisor $D$ in a complex manifold $M$ one means any loop in $\pi_{1}(M \backslash D)$ whose image in the homology group $H_{1}(M \backslash D)$ coincides with a fibre of the normal circle bundle of the smooth part reg $D$.

The next proposition provides a generalization of Lemma 3.4 in $\mathrm{Ka} 1$.

Proposition 3.1: Preserving the fundamental group under a modification. Let a pair $\left(M^{\prime}, E^{\prime}\right)$ be a pseudoaffine modification of a triple $(M, C, D)$ via a morphism $\sigma: M^{\prime} \rightarrow M$ where $M, M^{\prime}$ are complex manifolds, and let the divisors $D, E^{\prime}$ admit finite decompositions into irreducible components $D=\bigcup_{i=1}^{n} D_{i}$ resp. $E^{\prime}=\bigcup_{j=1}^{n^{\prime}} E_{j}^{\prime}$. Let $D_{i}^{*}=\sum_{j=1}^{n^{\prime}} m_{i j} E_{j}^{\prime}$. Assume that

(i) $\sigma\left(E_{j}^{\prime}\right) \cap \operatorname{reg} D_{i} \neq \emptyset$ as soon as $m_{i j}>0$.

Then the following statements (a) and (b) hold.

(a) If $(\alpha)$ the lattice vectors $b_{j}=\left(m_{1 j}, \ldots, m_{n j}\right) \in \mathbf{Z}^{n}, j=1, \ldots, n^{\prime}$, generate the lattice $\mathbf{Z}^{n}$, then $(\beta) \sigma_{*}: H_{1}\left(M^{\prime} ; \mathbf{Z}\right) \rightarrow H_{1}(M ; \mathbf{Z})$ is an isomorphism.

The converse is true if

(ii) $D_{i}$ is a principal divisor defined by a holomorphic function $f_{i}$ on $M$, i.e. $D_{i}=$ $f_{i}^{*}(0), i=1, \ldots, n$.

(b) Assume further that

(iii) there is a disjoint partition $\left\{1, \ldots, n^{\prime}\right\}=J_{1} \cup \ldots \cup J_{n}$ such that $D_{i}^{*}=\sum_{j \in J_{i}} m_{i j} E_{j}^{\prime} \neq$ $0, i=1, \ldots, n$.

Set $d_{i}=$ g.c.d. $\left(m_{i j} \mid j \in J_{i}\right), i=1, \ldots, n$.

Then $(\gamma) \sigma_{*}: \pi_{1}\left(M^{\prime}\right) \rightarrow \pi_{1}(M)$ is an isomorphism if $(\delta) d_{1}=\ldots=d_{n}=1$.

Under the condition (ii) the converse is also true. 
Proof. Let $\alpha_{i}$ be a vanishing loop of $D_{i}$ in $M, i=1, \ldots, n$, and $\beta_{j}$ be a vanishing loop of $E_{j}^{\prime}$ in $M^{\prime}, j=1, \ldots, n^{\prime}$. Then the kernel of the natural homomorphism $\pi_{1}(M \backslash D) \rightarrow$ $\pi_{1}(M)$ coincides with the minimal normal subgroup $H:=<<\alpha_{1}, \ldots, \alpha_{n}>>$ of the group $G:=\pi_{1}(M \backslash D)$ generated by $\alpha_{1}, \ldots, \alpha_{n}$, i.e. with the subgroup generated by the conjugacy classes of $\alpha_{1}, \ldots, \alpha_{n}$ (see e.g. [Za 2, (2.3.a)]). Similarly, the kernel of the natural homomorphism $\pi_{1}\left(M^{\prime} \backslash E^{\prime}\right) \rightarrow \pi_{1}\left(M^{\prime}\right)$ is the normal subgroup $H^{\prime}=<<$ $\beta_{1}, \ldots, \beta_{n^{\prime}}>>$ of the group $\pi_{1}\left(M^{\prime} \backslash E^{\prime}\right) \stackrel{\sigma_{*}}{\simeq} \pi_{1}(M \backslash D)=G$. Thus, $\pi_{1}(M) \simeq G / H$ and $\pi_{1}\left(M^{\prime}\right) \simeq G / H^{\prime}$. Moreover, $\sigma_{*}$ identifies $H^{\prime}$ with a subgroup of $H$, the surjection $\sigma_{*}: \pi_{1}\left(M^{\prime}\right) \longrightarrow \pi_{1}(M)$ coincides with the canonical surjection $G / H \longrightarrow G / H^{\prime}$, and hence, ker $\sigma_{*} \simeq H / H^{\prime}$. It follows that $\sigma_{*}: \pi_{1}\left(M^{\prime}\right) \rightarrow \pi_{1}(M)$ is an isomorphism iff $H=H^{\prime}$.

Proof of (a). Denote $G^{\prime}=[G, G]$, and let $\rho: G \longrightarrow G / G^{\prime} \simeq H_{1}(M \backslash D ; \mathbf{Z})$ be the canonical surjection. Set $\widetilde{H}=\rho(H), \widetilde{H}^{\prime}=\rho\left(H^{\prime}\right)$ and $\widetilde{\alpha}_{i}=\rho\left(\alpha_{i}\right), \widetilde{\beta}_{j}=\rho\left(\beta_{j}\right)$. Clearly, $\operatorname{ker}\left(\sigma_{*}: H_{1}\left(M^{\prime} ; \mathbf{Z}\right) \rightarrow H_{1}(M ; \mathbf{Z})\right) \simeq \widetilde{H} / \widetilde{H}^{\prime}$. In view of the condition $(i)$ we have $\widetilde{\beta}_{j}=\sum_{i=1}^{n} m_{i j} \widetilde{\alpha}_{i}, j=1, \ldots, n^{\prime}$. Under the condition $(\alpha)$, the elements $\widetilde{\alpha}_{1}, \ldots, \widetilde{\alpha}_{n}$ can be expressed in terms of $\widetilde{\beta}_{1}, \ldots, \widetilde{\beta}_{n^{\prime}}$, and so, $\widetilde{H}^{\prime}=\widetilde{H}$. This proves the implication $(\alpha)$ $\Longrightarrow(\beta)$.

Assuming the condition $(i i)$ we have an isomorphism $f_{*}: \widetilde{H} \stackrel{\simeq}{\longrightarrow} \mathbf{Z}^{n}$ where $f:=$ $\left(f_{1}, \ldots, f_{n}\right)$ which sends $\widetilde{\alpha}_{1}, \ldots, \widetilde{\alpha}_{n}$ to the standard generators of the lattice $\mathbf{Z}^{n}$ and identifies the subgroup $\widetilde{H^{\prime}}$ with the sublattice $\Lambda \subset \mathbf{Z}^{n}$ spanned by the vectors $b_{j}=$ $(f \circ \sigma)_{*}\left(\widetilde{\beta}_{j}\right), j=1, \ldots, n^{\prime}$. Therefore, $\widetilde{H}=\widetilde{H}^{\prime}$ iff $\Lambda=\mathbf{Z}^{n}$, that is, iff the condition $(\alpha)$ holds. This yields the converse implication $(\beta) \Longrightarrow(\alpha)$.

Proof of (b). Under the assumptions $(i)$ and $($ iii $)$ the image $\sigma_{*}\left(\beta_{J}\right) \in G$ where $j \in J_{i}$ is conjugate with the element $\alpha_{i}^{m_{i j}}$. Thus, $H^{\prime}=<<\beta_{1}, \ldots, \beta_{n^{\prime}}>>$

$=<<\alpha_{i}^{m_{i j}}\left|j \in J_{i}, i=1, \ldots, n>>=<<\alpha_{i}^{d_{i}}\right| i=1, \ldots, n>>$.

Therefore, the condition $(\delta)$ implies the coincidence $H=H^{\prime}$, and so, it implies $(\gamma)$. If $(i)$ - $($ iii $)$ hold, then, clearly, we have the implications $(\gamma) \Longrightarrow(\beta) \Longrightarrow(\alpha) \Longleftrightarrow$ $(\delta)$, and hence, $(\gamma) \Longrightarrow(\delta)$.

The next theorem and its corollary generalize Theorem 3.5 in $\mathrm{Ka} 1$ (see also Za 2, Thm. 5.1]).

\section{Theorem 3.1: Preserving the homology under a modification.}

Let a pair $\left(M^{\prime}, E^{\prime}\right)$ be a pseudoaffine modification of a triple $(M, C, D)$ via a morphism $\sigma: M^{\prime} \rightarrow M$ (see Definition 3.1). Suppose that

(i) $M, M^{\prime}$ are complex manifolds and $D, E^{\prime}$ are topological manifolds admitting finite decompositions into irreducible components $D=\sum_{i=1}^{n} D_{i}$ resp. $\quad E^{\prime}=\sum_{j=1}^{m} E_{j}^{\prime}$ where $m=n$ and $E_{i}^{\prime}=\sigma^{*}\left(D_{i}\right), i=1, \ldots, n$;

(ii) $\sigma\left(E_{i}\right) \cap \operatorname{reg} D_{i} \neq \emptyset, i=1, \ldots, n$.

Put $\tau=i \circ\left(\sigma \mid E^{\prime}\right): E^{\prime} \rightarrow D$ where $i: C \hookrightarrow D$ is the identical embedding. Then $\sigma_{*}: H_{*}\left(M^{\prime} ; \mathbf{Z}\right) \longrightarrow H_{*}(M ; \mathbf{Z})$ is an isomorphism iff

(iii) $\tau_{*}: H_{*}\left(E_{i}^{\prime} ; \mathbf{Z}\right) \longrightarrow H_{*}\left(D_{i} ; \mathbf{Z}\right)$ is an isomorphism for all $i=1, \ldots, n$.

Proof. Set $\breve{M}=M \backslash D, \breve{M}^{\prime}=M^{\prime} \backslash E^{\prime}$ and $\breve{\sigma}=\sigma \mid \breve{M}^{\prime}: \breve{M}^{\prime} \stackrel{\simeq}{\longrightarrow} \breve{M}$. Consider the following commutative diagram where the horizontal lines are exact homology sequences of pairs with $\mathbf{Z}$ - coefficients: 


$$
\begin{aligned}
& \ldots \longrightarrow H_{j+1}\left(M^{\prime}, \breve{M}^{\prime}\right) \longrightarrow H_{j}\left(\breve{M}^{\prime}\right) \longrightarrow H_{j}\left(M^{\prime}\right) \longrightarrow H_{j}\left(M^{\prime}, \breve{M}^{\prime}\right) \longrightarrow H_{j-1}\left(\breve{M}^{\prime}\right) \longrightarrow \ldots \\
& \downarrow(\sigma, \breve{\sigma})_{*} \quad \downarrow \breve{\sigma}_{*} \quad \downarrow \sigma_{*} \quad \downarrow(\sigma, \breve{\sigma})_{*} \quad \downarrow \breve{\sigma}_{*} \\
& \ldots \longrightarrow H_{j+1}(M, \breve{M}) \longrightarrow H_{j}(\breve{M}) \longrightarrow H_{j}(M) \longrightarrow H_{j}(M, \breve{M}) \longrightarrow H_{j-1}(\breve{M}) \longrightarrow \ldots
\end{aligned}
$$

Due to the Thom isomorphism, it can be replaced by the following one:

$$
\begin{aligned}
& \ldots \longrightarrow H_{j-1}\left(E^{\prime}\right) \longrightarrow H_{j}\left(\breve{M}^{\prime}\right) \longrightarrow H_{j}\left(M^{\prime}\right) \longrightarrow H_{j-2}\left(E^{\prime}\right) \longrightarrow H_{j-1}\left(\breve{M}^{\prime}\right) \longrightarrow \ldots \\
& \downarrow \tau_{*} \quad \simeq \breve{\sigma}_{*} \quad \downarrow \sigma_{*} \quad \simeq \downarrow \breve{\sigma}_{*} \\
& \ldots \longrightarrow H_{j-1}(D) \longrightarrow H_{j}(\breve{M}) \longrightarrow H_{j}(M) \longrightarrow H_{j-2}(D) \longrightarrow H_{j-1}(\breve{M}) \longrightarrow \ldots
\end{aligned}
$$

which is still commutative. Indeed, let $\Delta_{i}^{\prime}$ be a small complex disc in $M^{\prime}$ which meets $E_{i}^{\prime}$ transversally at its origin, and let this origin be a generic point of $E_{i}^{\prime}$. Then the image $\Delta_{i}=\sigma\left(\Delta_{i}^{\prime}\right)$ is a complex disc in $M$ transversal to reg $D_{i}$ at a generic point of $C_{i}$. Hence, for the Thom classes $u_{i}^{\prime} \in H^{2}\left(M^{\prime}, M^{\prime} \backslash E_{i}^{\prime}\right)$ of $E_{i}^{\prime}$ resp. $u_{i} \in H^{2}\left(M, M \backslash D_{i}\right)$ of $D_{i}$, uniquely defined by the conditions $u_{i}^{\prime}\left(\Delta_{i}^{\prime}\right)=1$ resp. $u_{i}\left(\Delta_{i}\right)=1$, we have $\sigma^{*}\left(u_{i}\right)=u_{i}^{\prime}, i=1, \ldots, n$. Recall that the Thom isomorphism $H_{j+2}\left(M^{\prime}, \breve{M}^{\prime}\right) \simeq H_{j}\left(E^{\prime}\right)$ resp. $H_{j+2}(M, \breve{M}) \simeq H_{j}\left(D^{\prime}\right)$ is defined as the cap-product with the Thom class $u^{\prime}=$ $\sum_{i=1}^{n} u_{i}^{\prime}$ resp. $u=\sum_{i=1}^{n} u_{i}: \quad \eta^{\prime} \mapsto u^{\prime} \cap \eta^{\prime}$ resp. $\eta \mapsto u \cap \eta$ (see e.g. [Do, VIII.11.21], MilSta]). Now it is easily seen that the diagram $(* *)$ is commutative.

It remains to note that by the Five Lemma, $\tau_{*}$ in $(* *)$ yields an isomorphism for all $j$ iff $\sigma_{*}: H_{j}\left(M^{\prime}\right) \rightarrow H_{j}(M)$ is an isomorphism for all $j$. The proof is completed.

\section{Remarks}

3.1. If $\sigma=\sigma_{C}$ is the blowup morphism with a smooth center $C$ contained in reg $D$, or otherwise, if $M^{\prime}$ is the affine modification $\Sigma_{I, f}(M)$ with the ideal $I$ admitting a regular system of generators started with $f$ (see Proposition $1.1(\mathrm{~d})$ ), then $\tau: E^{\prime} \rightarrow C$ is a smooth fibration over $C$ with a fibre $\mathbf{C}^{k}$, where $k=\operatorname{codim}_{D} C$, and so, the contraction $\sigma \mid E^{\prime}: E^{\prime} \rightarrow C$ is a homotopy equivalence. Thus, in this case $\tau_{*}: H_{*}\left(E^{\prime} ; \mathbf{Z}\right) \longrightarrow$ $H_{*}(D ; \mathbf{Z})$ is an isomorphism iff $i_{*}: H_{*}(C ; \mathbf{Z}) \longrightarrow H_{*}(D ; \mathbf{Z})$ is.

3.2. From the biholomorphism $\sigma: M^{\prime} \backslash E^{\prime} \rightarrow M \backslash D$ we get the equality for the Euler characteristics $e\left(M^{\prime}\right)-e(M)=e\left(E^{\prime}\right)-e(D)$ (see [Du]). In the situation as in the above remark we have $e\left(E^{\prime}\right)=e(C)$, and hence $e\left(M^{\prime}\right)-e(M)=e(C)-e(D)$.

\section{Corollary 3.1: Preserving contractibility under a modification.}

Suppose that the conditions ( $i$ ), ( $i$ i ) and (iii) of Theorem 3.1 are fulfilled. Then the pseudoaffine modification $M^{\prime}$ of $M$ is acyclic resp. contractible iff $M$ is.

Proof. The equivalence of acyclicity of $M^{\prime}$ and of $M$ follows immediately from Theorem 3.1.

By the Hurewicz and Whitehead Theorems [FoFu, (2.11.5), (2.14.2)], contractibility of $M$ resp. $M^{\prime}$ is equivalent to acyclicity and simply connectedness of $M$ resp. $M^{\prime}$. Notice that the conditions $(i),($ iii $)$ and $(\delta)$ of Proposition 3.1 are fulfilled. By (b) 
of this proposition, $\sigma: M^{\prime} \rightarrow M$ induces an isomorphism of the fundamental groups. Thus, $M^{\prime}$ is simply connected iff $M$ is, and the statement follows.

We give below several examples of applications of Corollary 3.1 .

Example 3.1: The tom Dieck-Petrie surfaces [EDP]. These are the smooth surfaces $X_{k, l}$ in $\mathrm{C}^{3}$ defined by the polynomials

$$
p_{k, l}=\frac{(x z+1)^{k}-(y z+1)^{l}-z}{z} \in \mathbf{C}[x, y, z]
$$

where $k, l \geq 2, \operatorname{gcd}(k, l)=1$. As in Example 2.3 above (see also Za 2, Example 6.1]) we have $X_{k, l}=\Sigma_{\rho, \Gamma_{k, l}}\left(\mathbf{C}^{2}\right)$ where $\Gamma_{k, l}=\left\{x^{k}-y^{l}=0\right\} \subset \mathbf{C}^{2}$ and $\rho=(1,1) \in \Gamma_{k, l}$. By Corollary 3.1, the surfaces $X_{k, l}$ are contractible.

Example 3.2: Russell's cubic is contractible. Recall (see Example 1.5) that the Russell cubic threefold $X=\left\{x+x^{2} y+z^{2}+t^{3}=0\right\} \subset \mathbf{C}^{4}$ is the affine modification of $\mathbf{C}^{3}$ along the divisor $2 D$, where $D:=\{x=0\} \subset \mathbf{C}^{3}$, with center at the ideal $I=\left(-x^{2}, x+z^{2}+t^{3}\right) \subset \mathbf{C}^{[3]}$ supported by the plane curve $C=\Gamma_{2,3}=\left\{x=z^{2}+t^{3}=\right.$ $0\} \subset D \simeq \mathbf{C}^{2}$. The exceptional divisor $E^{\prime}$ coincides with the book-surface $B=\{x=$ 0\} $\subset X^{\prime}, \quad B \simeq \mathbf{C} \times \Gamma_{2,3}$. Therefore, the condition ( $\left.i i i\right)$ of Theorem 3.1 is fulfilled. As well, the conditions $(i)$ and $(i i)$ hold, and so, by Corollary 3.1, $X$ is contractible. Moreover, by the Dimca-Ramanujam Theorem [Di 1, Ram] (see also [Za 2, Thm 4.2]), $X$ is diffeomorphic to $\mathbf{R}^{6}$.

Example 3.3: Modifying an affine space along a scroll. Let $M=f^{*}(0)$ be a smooth reduced analytic hypersurface in $\mathbf{C}^{n}$ where $f \in \mathcal{O}\left(\mathbf{C}^{n}\right)$, and let $f_{1}, \ldots, f_{k} \in$ $\mathcal{O}\left(\mathbf{C}^{n}\right)$ be holomorphic functions without common zeros on $M$. Consider the smooth analytic subvarieties

$$
D:=M \times \mathbf{C}^{k} \subset \mathbf{C}_{(\bar{x}, \bar{u})}^{n+k}=\mathbf{C}_{\bar{x}}^{n} \times \mathbf{C}_{\bar{u}}^{k} \quad \text { and } \quad C:=\{f(\bar{x})=0=g(\bar{x}, \bar{u})\} \subset \mathbf{C}_{(\bar{x}, \bar{u})}^{n+k}
$$

where $g(\bar{x}, \bar{u}):=\sum_{i=1}^{k} u_{i} f_{i} \in \mathcal{O}\left(\mathbf{C}^{n+k}\right)$. The natural embeddings $M \times \overline{0} \hookrightarrow C \hookrightarrow D$ being homotopy equivalences, by Corollary 3.1 and Remark 3.1, the pseudoaffine modification $X^{\prime}=\Sigma_{C, D}\left(\mathbf{C}^{n+k}\right)$ of $\mathbf{C}^{n+k}$ is a smooth contractible analytic hypersurface given in $\mathbf{C}_{(\bar{x}, \bar{u}, v)}^{n+k+1}$ by the equation $f(\bar{x}) v-g(\bar{x}, \bar{u})=0$ (cf. Example 1.4).

Since $M$ is supposed being smooth one may take, for instance, $k=n$ and $f_{i}=$ $\frac{\partial f}{\partial x_{i}}, i=1, \ldots, n ;$ then $C \simeq T M$ and $D \simeq T \mathbf{C}^{n} \mid M$.

The next example shows that in general, Corollary 3.1 does not hold if some of the conditions $(i)-($ iii $)$ of Theorem 3.1 are violated.

Example 3.4: Modifying with center at the singular locus of the divisor [Ka 1, Remark on p. 418]. Let $\sigma: X \rightarrow \mathbf{C}^{2}$ be a pseudoaffine modification of $\mathbf{C}^{2}$ along the cuspidal cubic $\Gamma:=\Gamma_{2,3}=\left\{x^{2}-y^{3}=0\right\}$ with center at the cusp $\overline{0} \in \Gamma$, that is, $\sigma$ is the restriction to $X:=\widehat{X} \backslash \Gamma^{\prime}$ of the blowup morphism $\widehat{\sigma}: \widehat{X} \rightarrow \mathbf{C}^{2}$ with center at the origin, whereas $\Gamma^{\prime} \subset \widehat{X}$ is the strict transform of $\Gamma$ in $\widehat{X}$. Then $X$ is neither simply connected nor acyclic. Indeed, set $X^{*}=X \backslash E^{\prime}=\widehat{X} \backslash\left(E \cup \Gamma^{\prime}\right)$ where $E \subset \widehat{X}$ is the exceptional divisor of $\widehat{\sigma}$, and let $\beta \in H_{1}\left(X^{*} ; \mathbf{Z}\right)$ be a vanishing loop of $\Gamma^{\prime}$ in $X^{*}$. It is easily seen that $\sigma_{*}(\beta)=2 \alpha$ where $\alpha \in H_{1}\left(\mathbf{C}^{2} \backslash \Gamma ; \mathbf{Z}\right) \simeq \mathbf{Z}$ is a vanishing loop of $\Gamma$ in $\mathbf{C}^{2}$. In virtue of the isomorphism $\sigma \mid X^{*}: X^{*} \stackrel{\simeq}{\longrightarrow} \mathbf{C}^{2} \backslash \Gamma$ and of the exact sequence

$$
\mathbf{0} \longrightarrow<\beta>\longrightarrow H_{1}\left(X^{*} ; \mathbf{Z}\right) \longrightarrow H_{1}(X ; \mathbf{Z}) \longrightarrow \mathbf{0}
$$


(cf. Lemma 7.3 below) we have

$$
H_{1}(X ; \mathbf{Z}) \simeq H_{1}\left(X^{*} ; \mathbf{Z}\right) /<\beta>\simeq H_{1}\left(\mathbf{C}^{2} \backslash \Gamma ; \mathbf{Z}\right) /<2 \alpha>\simeq \mathbf{Z} / 2 \mathbf{Z}
$$

Remark 3.3. Notice that the proper transform $\Gamma^{\mathrm{pr}}$ of $\Gamma$ in $\widehat{X}$ (see Remark 1.3) coincides with the union $E \cup \Gamma^{\prime}$, and so, it differs from the strict transform $\Gamma^{\prime}$. In turn, $X$ differs from the affine modification $\Sigma_{I, f}\left(\mathbf{C}^{2}\right) \simeq \mathbf{C}^{2} \backslash \Gamma$ where $I:=\left(x^{2}-y^{3}, x, y\right)$. Indeed, the blow up $Z=\widehat{X}$ can be given in $\mathbf{C}_{(x, y)}^{2} \times \mathbf{P}_{(u: v: w)}^{2}$ by the equations $x v-y u=0, w=$ $x u-y^{2} v$, and hence, $\Gamma^{\mathrm{pr}}=\{w=0\}$ contains the exceptional divisor $10 E=\{x=y=0\}$.

\section{Topology of the hypersurfaces $u v=p\left(x_{1}, \ldots, x_{k}\right)$}

Notation. Let $X=X(p) \subset \mathbf{C}^{k+2}$ be the irreducible hypersurface given by the equation $u v=p(\bar{x})$ where $\bar{x}=\left(x_{1}, \ldots, x_{k}\right) \in \mathbf{C}^{k}, k \geq 1$, and $p \in \mathbf{C}^{[k]}$, $\operatorname{deg} p>0$. For $c \in \mathbf{C}$ denote $U_{c}=X \cap\{u=c\}, V_{c}=X \cap\{v=c\}$, and $X_{0}=p^{-1}(0) \subset \mathbf{C}^{k}$. We also regard $X_{0}$ as the subvariety of $X$ given in $\mathbf{C}^{k+2}$ by the equations $u=v=p(\bar{x})=0$.

Remark 4.1. The variety $X(p)$ is the affine modification of $\mathbf{C}_{\bar{x}, u}^{k+1}$ along the hyperplane $D_{u}=\{u=0\}$ with center $I=(p, u) \subset \mathbf{C}^{[k+1]}$ and with the exceptional divisor $U_{0} \subset$ $X(p)$ (see Example 1.4). If the divisor $p^{*}(0)$ is reduced, then $X(p)=\Sigma_{X_{0}, D_{u}}\left(\mathbf{C}^{k+1}\right)$.

The following lemma is easy, and we omit the proof.

Lemma 4.1. (a) There are natural isomorphisms $X \backslash U_{0} \simeq X \backslash V_{0} \simeq \mathbf{C}^{k} \times \mathbf{C}^{*}, U_{0} \simeq$ $V_{0} \simeq X_{0} \times \mathbf{C}$, and $U_{0} \cap V_{0}=X_{0}$. In particular, $e(X)=e\left(X_{0}\right)$.

(b) $X$ is smooth iff $p^{*}(0)$ is a smooth reduced (not necessarily irreducible) divisor in $\mathbf{C}^{k}$ (supported by $X_{0}$ ). Furthermore, if the divisor $p^{*}(0)$ is reduced, then we have

$$
\operatorname{sing} X=\operatorname{sing} U_{0} \cap \operatorname{sing} V_{0}=\operatorname{sing} X_{0} \quad \text { and } \quad \operatorname{sing} U_{0} \simeq\left(\operatorname{sing} X_{0}\right) \times \mathbf{C} \simeq \operatorname{sing} V_{0}
$$

Proposition 4.1. Assume that $X$ is smooth. Then $X$ is simply connected, and the embedding $X_{0} \hookrightarrow X$ induces an isomorphism of the reduced homology groups $\tilde{H}_{*}(X ; \mathbf{Z}) \simeq$ $\tilde{H}_{*-2}\left(X_{0} ; \mathbf{Z}\right)$.

Proof. Since $X$ is assumed being smooth, by Lemma 4.1(b), $X_{0}$ is smooth and reduced. By Lemma 4.1(a), we have that $\pi_{1}\left(X \backslash U_{0}\right) \simeq \pi_{1}\left(\mathbf{C}^{k} \times \mathbf{C}^{*}\right) \simeq \mathbf{Z}$. The kernel of the natural surjection $i_{*}: \pi_{1}\left(X \backslash U_{0}\right) \longrightarrow \pi_{1}(X)$ is generated, as a normal subgroup, by vanishing loops, say $\alpha_{i}$, of the irreducible components $U_{0}^{(i)} \simeq X_{0}^{(i)} \times \mathbf{C}$ of $U_{0}$ where $X_{0}^{(i)} \subset \mathbf{C}^{k}, i=1, \ldots, l$, are the irreducible components of $X_{0}$ (see e.g. [Za 2, (2.3.a)]).

Denote $\rho=\left(u \mid\left(X \backslash U_{0}\right)\right)_{*}: \pi_{1}\left(X \backslash U_{0}\right) \rightarrow \pi_{1}\left(\mathbf{C}^{*}\right) \simeq \mathbf{Z}$. Fix a generator $t$ of the group $\pi_{1}\left(X \backslash U_{0}\right) \simeq \mathbf{Z}$ such that $\rho(t)=1$. Clearly, $\rho\left(\alpha_{i}\right)= \pm 1 \in \mathbf{Z}=\pi_{1}\left(\mathbf{C}^{*}\right)$; that is, $\alpha_{i}= \pm t, i=1, \ldots, l$, and hence, Ker $i_{*}=\left\langle\alpha_{1}, \ldots, \alpha_{l}\right\rangle=\mathbf{Z}$. Thus, $\pi_{1}(X)=\mathbf{1}$, as claimed.

\footnotetext{
${ }^{10}$ More generally, one can prove the following:

If the center $C$ of a blow up of a manifold $X$ is smooth, reduced and it is contained in the singular locus of a reduced divisor $D_{f}$, then the proper transform $D_{f}^{\mathrm{pr}}$ contains the exceptional divisor $E$ of the blow up.
} 
Therefore, also $H_{1}(X ; \mathbf{Z})=0$. Due to Lemma 1.1(a), from the exact homology sequence of the pair $\left(X, X \backslash U_{0}\right)$ we obtain the isomorphisms of the $\mathbf{Z}$ - homology groups: $H_{i}(X) \simeq H_{i}\left(X, X \backslash U_{0}\right)$ for all $i \geq 3$. In view of the Thom isomorphism (denoted below by $\tau$ ) this yields the isomorphisms

$$
H_{i}(X) \simeq H_{i}\left(X, X \backslash U_{0}\right) \stackrel{\tau}{\simeq} H_{i-2}\left(U_{0}\right) \simeq H_{i-2}\left(X_{0}\right) \forall i \geq 3 .
$$

For $i=2$ we get

$$
\begin{aligned}
0= & H_{2}\left(X \backslash U_{0}\right) \rightarrow H_{2}(X) \rightarrow H_{2}\left(X, X \backslash U_{0}\right) \stackrel{\tau}{\simeq} H_{0}\left(U_{0}\right) \\
& \simeq H_{0}\left(X_{0}\right) \stackrel{\partial_{*}}{\longrightarrow} \mathbf{Z} \simeq H_{1}\left(X \backslash U_{0}\right) \rightarrow 0=H_{1}(X) .
\end{aligned}
$$

Since $X_{0}$ is a smooth reduced divisor, the number $l=\operatorname{deg} p$ of its irreducible components coincides with the number $b_{0}\left(X_{0}\right)$ of its connected components. Recall (see e.g. MilSta]) that the Thom isomorphism $\tau: H_{0}\left(U_{0}\right) \stackrel{\simeq}{\longrightarrow} H_{2}\left(X, X \backslash U_{0}\right)$ to each point $P \in U_{0}$ associates the relative homology class $\left[\Delta_{P}\right] \in H_{2}\left(X, X \backslash U_{0}\right)$ of a disc $\Delta_{P} \subset X$ centered at $P$ and transversal to $U_{0}$; furthermore, $\partial_{*}\left[\Delta_{P}\right]=\left[\partial \Delta_{P}\right] \in H_{1}\left(X \backslash U_{0}\right)$. Thus, for $\beta=\left(\beta_{1}, \ldots, \beta_{l}\right) \in H_{2}\left(X, X \backslash U_{0}\right) \simeq H_{0}\left(X_{0}\right) \simeq \mathbf{Z}^{l}$ we have $\partial_{*}(\beta)=\sum_{i=1}^{l} \beta_{i} \in$ $H_{1}\left(X \backslash U_{0}\right) \simeq \mathbf{Z}$. It follows that $H_{2}(X) \simeq \operatorname{ker} \partial_{*} \simeq \mathbf{Z}^{b_{0}\left(X_{0}\right)-1} \simeq \tilde{H}_{0}\left(X_{0}\right)$; in particular, $H_{2}(X)=0$ iff $X_{0}$ is irreducible. Since $X$ is irreducible, we conclude that $\tilde{H}_{*}(X) \simeq \tilde{H}_{*-2}\left(X_{0}\right)$, and the second statement of the proposition follows.

Corollary 4.1. Suppose that $X$ is smooth. Then $X$ is contractible iff $X_{0}$ is acyclic; actually, in this case $X$ is diffeomorphic to $\mathbf{R}^{2 k+2}$.

The last statement follows from the Dimca-Ramanujam Theorem [Di 1, Ram] (see also [Za 2, Thm 4.2]) for $k \geq 2$, and for $k=1$ it follows from the next proposition.

Proposition 4.2. (a) Let $k=1$. The following conditions are equivalent:

(i) $X \simeq \mathrm{C}^{2}$;

(ii) $X \subset \mathbf{C}^{3}$ is a smooth acyclic surface;

(iii) $\operatorname{deg} p=1$.

(b) Let $k=2$. The following conditions are equivalent:

(i) the embedding $X \hookrightarrow \mathbf{C}^{4}$ is rectifiable;

(ii) $X \simeq \mathbf{C}^{3}$;

(iii) $X \subset \mathbf{C}^{4}$ is a smooth acyclic 3-fold;

(iv) the divisor $p^{*}(0)$ is reduced and $X_{0} \simeq \mathbf{C}$;

(v) the polynomial $p \in \mathbf{C}^{[2]}$ is equivalent to a linear one, i.e. for some $\alpha \in A u t \mathbf{C}^{2}$ we have $p \circ \alpha=q$ where $q\left(x_{1}, x_{2}\right)=x_{1}$.

Proof. (a) Let $k=1$. The implications ( $i i i) \Longrightarrow(i) \Longrightarrow($ ii $)$ are clear. Conversely, by Lemma 4.1(a),(b), under the assumption $(i i)$ we have $1=e(X)=e\left(X_{0}\right)=\operatorname{deg} p$. This proves (a).

(b) The theorem of Abhyankar-Moh and Suzuki [AM, Suz] asserts the equivalence ( $i v$ ) $\Longleftrightarrow(v)$. The implications $(v) \Longrightarrow(i) \Longrightarrow(i i) \Longrightarrow($ iii $)$ are easy; (iii $) \Longrightarrow$ ( iv ) follows from Lemma 4.1(b) and Proposition 4.1, and so, we are done.

Remark 4.2. However, starting with $k=3$ the equivalences of Proposition 4.2 fail; see Examples 6.1 and 6.2 below. 
A generalization. More generally, consider the variety $X=X\left(p_{1}, \ldots, p_{m}\right) \subset \mathbf{C}^{k+m+1}$ given by a system of equations $u v_{i}=p_{i}(\bar{x}), i=1, \ldots, m$ where $\bar{x}=\left(x_{1}, \ldots, x_{k}\right) \in$ $\mathbf{C}^{k}, k \geq m \geq 1$, and $p_{i} \in \mathbf{C}^{[k]} \backslash \mathbf{C}, i=1, \ldots, m$. This variety $X$ is the affine modification of $\mathbf{C}_{(\bar{x}, u)}^{k+1}$ along the hyperplane $D_{u}=\{u=0\}$ with center at the ideal $I=\left(p_{1}, \ldots, p_{m}, u\right) \subset \mathbf{C}^{[k+1]}$ supported by the affine variety $X_{0}:=\left\{p_{1}(\bar{x})=\ldots=\right.$ $\left.p_{m}(\bar{x})=0\right\} \subset D_{u} \simeq \mathbf{C}^{k}$. Clearly, $X$ is irreducible iff $X_{0}$ is a set-theoretic complete intersection, i.e. all its irreducible components are codimension $m$ subvarieties of $\mathbf{C}^{k}$, which will be always assumed in the sequel. Under this assumption most of the results proved above for $m=1$ remain true in this more general setting.

Proposition 4.3. (a) Denote by $U_{0}$ the hypersurface in $X=X\left(p_{1}, \ldots, p_{m}\right)$ given by the equation $u=0$. Then we have $U_{0} \simeq X_{0} \times \mathbf{C}^{m}$ and $X \backslash U_{0} \simeq \mathbf{C}^{*} \times \mathbf{C}^{m}$. In particular, $e(X)=e\left(X_{0}\right)$.

(b) Assume further that

(i) $X_{0}$ is the ideal-theoretic complete intersection of the divisors $p_{i}^{*}(0), i=1, \ldots, m$, that is, $I\left(X_{0}\right)=\left(p_{1}, \ldots, p_{m}\right)$.

Then we have $\operatorname{sing} X_{0} \subset \operatorname{sing} X \subset \operatorname{sing} U_{0} \simeq\left(\operatorname{sing} X_{0}\right) \times \mathbf{C}^{m}$. In particular, $X$ is smooth iff $X_{0}$ is.

(c) Suppose that the assumption (i) is fulfilled and $X$ is smooth. Then we have $\pi_{1}(X)=$ 1 and $\tilde{H}_{*}(X ; \mathbf{Z}) \simeq \tilde{H}_{*-2}\left(X_{0} ; \mathbf{Z}\right)$. In particular, $X$ is contractible (and, moreover, diffeomorphic to $\mathbf{R}^{2 k+2}$ for $k \geq 2$ ) iff $X_{0}$ is acyclic.

The proof goes exactly in the same way as before, and so, we leave it to the reader.

Remark 4.3. Presumably, even being diffeomorphic to the affine space, the variety $X=X\left(p_{1}, \ldots, p_{m}\right)$ is not, in general, isomorphic to $\mathbf{C}^{k+1}$ for $k \geq 3$, and so, it should provide an exotic algebraic structure on $\mathbf{C}^{k+1}$ (see e.g. [Za 2]). But at present we have no invariant available to distinguish $X$ from the affine space (see Remark 5.2 below).

Suppose that, indeed, for a certain smooth acyclic complete intersection $X_{0} \subset \mathbf{C}^{k}$ the variety $X=X\left(p_{1}, \ldots, p_{m}\right)$ is not isomorphic to $\mathbf{C}^{k+1}$. Then we would have an example showing that Miyanishi's characterization of the affine 3-space $\mathbf{A}_{k}^{3}$ [Miy] does not hold any more in higher dimensions. Indeed, by Proposition 4.3, the varieties $X$ and $U_{0} \simeq X_{0} \times \mathbf{C}^{m}$ being smooth and acyclic we have $e(X)=e\left(U_{0}\right)=1, \quad X \backslash U_{0} \simeq \mathbf{C}^{*} \times \mathbf{C}^{k}$, the algebras $\mathbf{C}[X]$ and $\mathbf{C}\left[U_{0}\right]$ are UFD and have only constants as the units (see e.g. Ka 1, Prop. 3.2]). Thus, all the assumptions of the Miyanishi Theorem are fulfilled, whereas $X \not \mathbf{C}^{k+1}$.

Further, if for a certain codimension $m$ smooth acyclic complete intersection $X_{0} \subset \mathbf{C}^{k}$ non-isomorphic to $\mathbf{C}^{k-m}$ the variety $X$ were isomorphic to $\mathbf{C}^{k+1}$ this would answer in negative, alternatively, either to the Zariski Cancellation Problem 11 or to the AbhyankarSathaye Embedding Problem 12 . Indeed, the former happens if the hypersurface $U_{0}=$ $X_{0} \times \mathbf{C}^{m} \subset X$ is isomorphic to $\mathbf{C}^{k}$ (which is only possible if $X_{0}$ was contractible). Otherwise, the latter takes place since $U_{0}$ is the zero fibre of the polynomial $u \mid X \in$

\footnotetext{
${ }^{11}$ In the particular case when $k=3$ and $X_{0} \simeq \mathbf{C}$ is a complete intersection given by $p_{1}(\bar{x})=p_{2}(\bar{x})=$ 0 in $\mathbf{C}^{3}$, the smooth contractible 4 -folds $X=X\left(p_{1}, p_{2}\right) \subset \mathbf{C}^{5}$ were studied, in algebraic fashion, in [As] as potential counterexamples to the Zariski Cancellation Problem. Indeed, in [As] an isomorphism $X \times \mathbf{C} \simeq \mathbf{C}^{5}$ was established.

${ }^{12} \mathrm{Cf}$. also Remark 6.2 below for another conjectural counterexample to the Abhyankar-Sathaye Embedding Problem.
} 
$\mathbf{C}[X] \simeq \mathbf{C}^{[k+1]}$ with all other fibres $U_{c}, c \neq 0$, isomorphic to $\mathbf{C}^{k}$. Observe that due to the Miyanishi-Sugie and Fujita Cancellation Theorem, for $k-m \leq 2$ only the second possibility might happen.

Formally, there is also a possibility that $X \not \mathbf{C}^{k+1}$ whereas $U_{0} \simeq \mathbf{C}^{k}$. In that case we would have an example of an exotic $\mathbf{C}^{n}, n=k+1 \geq 4$, fibered by the affine spaces $U_{c} \simeq \mathbf{C}^{n-1}$.

We may enlarge our collection of contractible affine varieties passing to ramified cyclic coverings over $X=X\left(p_{1}, \ldots, p_{m}\right)$, as follows.

Proposition 4.4. (a) Suppose that the variety $X=X\left(p_{1}, \ldots, p_{m}\right)$ as in Proposition 4.3. (c) above is smooth and contractible. Then for any $n \in \mathbf{N}$ the variety $X_{n} \subset \mathbf{C}^{k+m+1}$ given by the system of equations $u^{n} v_{i}=p_{i}(\bar{x}), i=1, \ldots, m$, is smooth and contractible, too.

(b) For a sequence of integers $s_{0}, \ldots, s_{m} \in \mathbf{N}$ such that $\operatorname{gcd}\left(s_{i}, s_{j}\right)=1$ for all $i \neq j$, consider the variety

$$
Y=Y_{s_{0}, \ldots, s_{m}}\left(p_{1}, \ldots, p_{m}\right):=\left\{u^{s_{0}} v_{i}^{s_{i}}=p_{i}(\bar{x}), i=1, \ldots, m\right\} \subset \mathbf{C}^{k+m+1}
$$

where $p_{i} \in \mathbf{C}^{[k]}, i=1, \ldots, m$. Suppose that the following conditions are fulfilled:

(i) $p:=p_{1} \cdot \ldots \cdot p_{m}$ is a prime decomposition, and $D:=p^{*}(\overline{0})$ is a reduced simple normal crossing divisor in $\mathbf{C}^{k}$;

(ii) the divisor $D_{i}:=D_{p_{i}}$ is $\mathbf{Z}_{q}$ - acyclic for any prime divisor $q$ of $s_{i}, i=1, \ldots m$, and $X_{0}=\cap_{i=1}^{m} D_{i} \subset \mathbf{C}^{k}$ is a smooth acyclic complete intersection;

(iii) the group $\pi_{1}\left(\mathbf{C}^{k} \backslash D\right)$ is abelian (and hence, isomorphic to $\mathbf{Z}^{m}$ ).

Then $Y$ is a smooth contractible variety; it is diffeomorphic to $\mathbf{R}^{2 k+2}$ if $k \geq 2$.

Proof. (a) The variety $X_{n}$ is a cyclic covering of $X$ ramified to order $n$ on $U_{0}$ with the covering morphism $\rho: X_{n} \rightarrow X, \rho:(\bar{x}, u, \bar{v}) \longmapsto\left(\bar{x}, u^{n}, \bar{v}\right)$. By Proposition 4.3(c), the ramification divisor $U_{0}=X_{0} \times \mathbf{C}^{m}$ is acyclic, the fundamental group of its complement $\pi_{1}\left(X \backslash U_{0}\right) \simeq \mathbf{Z}$ is abelian, and the regular function $u \mid\left(X \backslash U_{0}\right)$ where $X \backslash U_{0} \simeq \mathbf{C}^{*} \times \mathbf{C}^{k}$, is a quasi-invariant of weight 1 of the natural $\mathbf{C}^{*}-$ action. Now the assertion of $(a)$ follows from Theorem $\mathrm{A}$ in [Ka 3] (see also [Za 2, Thm. 7.1]).

(b) Denote $V_{0}^{(i)}=X \cap\left\{v_{i}=0\right\}, i=1, \ldots, m$ where $X=X\left(p_{1}, \ldots, p_{m}\right)$. The morphism $\mathbf{C}^{k+m+1} \rightarrow \mathbf{C}^{k+m+1}, \quad\left(\bar{x}, u, v_{1}, \ldots, v_{m}\right) \longmapsto\left(\bar{x}, u^{s_{0}}, v_{1}^{s_{1}}, \ldots, v_{m}^{s_{m}}\right)$, restricted to $Y$ makes $Y$ a multi-cyclic covering of $X$ branched to order $s_{0}$ over $U_{0}$ resp. to order $s_{i}$ over $V_{0}^{(i)}, i=1, \ldots, m$. Thus, we may use Theorem 8.1 in Za 2 which provides conditions to guarantee contractibility of a multi-cyclic covering over a contractible manifold. To see that these conditions are satisfied, first of all, we observe that the function $u$ resp. $v_{i}, i=$ $1, \ldots, m$, is a quasi-invariant of weight 1 resp. -1 of the $\mathbf{C}^{*}-\operatorname{action}(\lambda,(\bar{x}, u, \bar{v})) \longmapsto$ $\left(\bar{x}, \lambda u, \lambda^{-1} \bar{v}\right)$ on $\mathbf{C}^{k+m+1}$ which leaves the variety $X$ invariant.

Further, the hypersurface $U_{0} \simeq X_{0} \times \mathbf{C}^{m}$ is smooth and acyclic since $X_{0}$ is. For each $i=1, \ldots, m$ the hypersurface $V_{0}^{(i)}$ is the affine modification of the smooth variety $D_{i} \times \mathbf{C}$ along the divisor $D_{i}$ with center $X_{0} \subset D_{i}$ and with the exceptional divisor $E_{i}:=U_{0} \cap V_{0}^{(i)} \simeq X_{0} \times \mathbf{C}^{m-1}$ via the morphism $\sigma_{i}: V_{0}^{(i)} \rightarrow D_{i} \times \mathbf{C}$ which is the restriction to $V_{0}^{(i)}$ of the projection $\pi: \mathbf{C}^{k+m+1} \rightarrow \mathbf{C}^{k+1}, \pi(\bar{x}, u, \bar{v})=(\bar{x}, u)$ (see Corollary 2.1). 
Notice that the proof of Theorem 3.1 on preservation of the homology under a modification goes equally for the $\mathbf{Z}_{p}$ - homology groups. In virtue of the condition (ii) above, by this Theorem, the smooth hypersurface $V_{0}^{(i)} \subset X$ is $\mathbf{Z}_{q}$ - acyclic for any prime divisor $q$ of $s_{i}, i=1, \ldots, m$.

We have an isomorphism $X^{*}:=X \backslash\left(U_{0} \cup V_{0}^{(i)} \cup \ldots \cup V_{0}^{(i)}\right) \simeq\left(\mathbf{C}^{k} \backslash D\right) \times \mathbf{C}^{*}$. Hence by the condition (iii), the fundamental group $\pi_{1}\left(X^{*}\right)$ is abelian. Now all the assumptions of Theorem 8.1 in [Za 2] are verified. By this theorem, $Y$ is contractible.

Corollary 4.2. Let $X_{0}=p^{*}(0), p \in \mathbf{C}[k]$, be a smooth reduced acyclic hypersurface in $\mathbf{C}^{k}$. Then for any $n \in \mathbf{N}$ the hypersurface $X_{n}:=\left\{u^{n} v=p(\bar{x})\right\}$ in $\mathbf{C}^{k+2}$ is smooth and contractible.

If, furthermore, $\pi_{1}\left(\mathbf{C}^{k} \backslash X\right) \simeq \mathbf{Z}$, then for any relatively prime integers $s_{0}, s_{1} \in \mathbf{N}$ the hypersurface $Y_{s_{0}, s_{1}}:=\left\{u^{s_{0}} v^{s_{1}}=p(\bar{x})\right\}$ in $\mathbf{C}^{k+2}$ is smooth and contractible.

Remark 4.4. For instance, one may take as $X_{0}$ the tom Dieck-Petrie surface $X_{k, l} \subset \mathbf{C}^{3}$ (see Example 3.1). Indeed, it is easily seen that it satisfies all the conditions of Corollary 4.2 .

\section{$5 \quad \mathbf{C}_{+}-$actions on the hypersurfaces $u v=p\left(x_{1}, \ldots, x_{k}\right)$}

This section is devoted to the proof of the following theorem.

The Transitivity Theorem. Let $X=\{u v-p(\bar{x})=0\} \subset \mathbf{C}^{k+2}$ where $k \geq 2$ and $p \in \mathbf{C}^{[k]} \backslash \mathbf{C}$. Then the automorphism group Aut $X$ acts $m$-transitively on $X \backslash \operatorname{sing} X$ for any $m \in \mathbf{N}$.

We keep all the notation from Section 4. Set $\sigma_{i}=\pi_{i} \mid X, i=1,2$ where $\pi_{i}: \mathbf{C}^{k+2} \rightarrow$ $\mathbf{C}^{k+1}, \pi_{1}:(\bar{x}, u, v) \longmapsto(\bar{x}, v)$, and $\pi_{2}:(\bar{x}, u, v) \longmapsto(\bar{x}, u)$, are the canonical projections. Then $\sigma_{1}: X \rightarrow \mathbf{C}^{k+1}=\mathbf{C}_{\bar{x}}^{k} \times \mathbf{C}_{v}$ resp. $\sigma_{2}: X \rightarrow \mathbf{C}^{k+1}=\mathbf{C}_{\bar{x}}^{k} \times \mathbf{C}_{u}$ is the affine modification of $\mathbf{C}^{k+1}$ along the hyperplane $D_{v}=\{v=0\}$ resp. $D_{u}=\{u=0\}$ with center色 $I_{1}=(p, v) \subset \mathbf{C}^{[k+1]}$ resp. $I_{2}=(p, u) \subset \mathbf{C}^{[k+1]}$ and with the exceptional divisor $V_{0} \subset X$ resp. $U_{0} \subset X$ (see Example 1.4).

Concretizing Corollaries 2.2 and 2.3 in our setting we obtain the following statement.

Lemma 5.1. Let $\varphi: \mathbf{C}_{+} \times \mathbf{C}^{k+1} \rightarrow \mathbf{C}^{k+1}$ resp. $\varphi: \mathbf{C}^{k+1} \rightarrow \mathbf{C}^{k+1}$ be a regular $\mathbf{C}_{+}$-action on $\mathbf{C}^{k+1}=\mathbf{C}_{\bar{x}}^{k} \times \mathbf{C}_{v}$ resp. an automorphism of $\mathbf{C}^{k+1}=\mathbf{C}_{\bar{x}}^{k} \times \mathbf{C}_{v}$. Suppose that $\varphi$ leaves the subvarieties $D_{v}=\{v=0\}$ and $X_{0}$ invariant. Then there is a unique regular $\mathbf{C}_{+}$-action $\hat{\varphi}$ on $X$ resp. an automorphism $\hat{\varphi}$ of $X$ which leaves the hypersurface $V_{0}$ invariant and such that the restriction $\hat{\varphi} \mid\left(X \backslash V_{0}\right)$ coincides with $\sigma_{1}^{-1} \varphi \sigma_{1}$.

Notation. Let $G_{1}$ resp. $G_{2}$ be the subgroup of the group Aut $\mathbf{C}^{k+1}$ where $\mathbf{C}^{k+1}=$ $\mathbf{C}_{\bar{x}}^{k} \times \mathbf{C}_{v}$ resp. $\mathbf{C}^{k+1}=\mathbf{C}_{\bar{x}}^{k} \times \mathbf{C}_{u}$, generated by all the $\mathbf{C}_{+}-$subgroups $T$ of Aut $\mathbf{C}^{k+1}$ such that the function $v$ resp. $u$ is a $T$-invariant, and the restriction of $T$ to the invariant hyperplane $D_{v}$ resp. $D_{u}$ leaves the subvariety $X_{0}$ invariant. Denote $\widehat{G}_{i}, i=$ 1,2 , the subgroup of the group Aut $X$ which corresponds to $G_{i}$ in view of Lemma 5.1, and let $\widehat{G} \subset$ Aut $X$ be the subgroup generated by $\widehat{G}_{1}$ and $\widehat{G}_{2}$. Notice that $\widehat{G}_{2}=$ $\varepsilon \widehat{G}_{1} \varepsilon^{-1}$ where $\varepsilon \in$ Aut $X, \varepsilon:(\bar{x}, u, v) \longmapsto(\bar{x}, v, u)$.

\footnotetext{
${ }^{13}$ with center $C_{1}=X_{0} \subset D_{v}$ resp. $C_{2}=X_{0} \subset D_{u}$ if the divisor $p^{*}(0)$ is reduced.
} 
The Transitivity Theorem can be precised as follows.

Theorem 5.1. The group $\widehat{G}$ acts $m$ - transitively on $X \backslash \operatorname{sing} X$ for any $m \in \mathbf{N}$.

Remarks . 5.1. For $k=1$ a description of the automorphism group Aut $X$ of the surface $X=X_{p} \subset \mathbf{C}^{3}$ was given in [ML 2]. It follows from that description that the action of Aut $X$ on $X$ is transitive, but not 2-transitive.

5.2. Recall [KaML 1, Za 2, (9.2)] that the Makar-Limanov invariant of an algebra $A$ over $\mathbf{C}$ is the subalgebra $\mathrm{ML}(A) \subset A$ which consists of all the elements invariant under every $\mathbf{C}_{+}-$subgroup of the automorphism group Aut $A$; or, which is the same, $\operatorname{ML}(A)=\bigcap_{\partial \in \operatorname{LND}(A)}$ ker $\partial$ (see Definition 2.2). From Theorem 5.1 it follows that for the algebra $A=\mathbf{C}[X]$ where $X$ is as above, this invariant is trivial, i.e. $\operatorname{ML}(A)=\mathbf{C}$. This is also true for $k=1$; see ML 2]. The problem arises to find a substitution of the Makar-Limanov invariant which would permit to distinguish the varieties $X=X(p)$ up to isomorphism, especially those diffeomorphic to the affine spaces.

The proof of Theorem 5.1 is based on Lemmas 5.2 - 5.6 below. In the next lemma for a class of $\mathbf{C}_{+}-$actions $\varphi=\varphi_{\partial}$ on $\mathbf{C}^{k+1}$ which preserve the decomposition $\mathbf{C}^{k+1}=$ $\mathbf{C}_{\bar{x}}^{k} \times \mathbf{C}_{v}$ we specify the lifts $\hat{\varphi} \in$ Aut $X$ of $\varphi$, cf. Lemma 5.1.

Lemma 5.2. Let $\delta$ be an LND of the polynomial algebra $\mathbf{C}^{[k]}=\mathbf{C}\left[x_{1}, \ldots, x_{k}\right]$, and let $q \in \mathbf{C}[z]$ be a degree $d$ polynomial with the roots $z_{1}=0, z_{2}, \ldots, z_{m}$ where $z_{i} \neq z_{j}$ for $i \neq j$. Then

(a) the formulas

$$
\partial\left(x_{i}\right)=q(v) \delta\left(x_{i}\right), i=1, \ldots, k, \quad \partial(v)=0
$$

define an LND $\partial=\partial_{\delta, q}$ on $\mathbf{C}^{[k+1]}=\mathbf{C}\left[x_{1}, \ldots, x_{k}, v\right]$. The associated $\mathbf{C}_{+}-$action $\varphi_{\partial} \subset G_{1}$ on $\mathbf{C}^{k+1}$ fixes each point of the hyperplanes $D_{j}=\left\{v=z_{j}\right\}, j=1, \ldots, m$.

(b) The corresponding lifted $L N D \widehat{\partial}$ on $A=\mathbf{C}[X]$ is the restriction to $X$ of the LND on $\mathbf{C}^{[k+2]}=\mathbf{C}[\bar{x}, u, v]$ (denote it also by $\widehat{\partial}$ ) given by the formulas

$$
\widehat{\partial}\left(x_{j}\right)=\partial\left(x_{j}\right), \quad \widehat{\partial}(v)=\partial(v)=0, \quad \widehat{\partial}(u)=\frac{1}{v} \partial(p(\bar{x}))=\frac{q(v)}{v} \delta p(\bar{x}) .
$$

Furthermore, the fixed point set Fix $\hat{\varphi}_{\partial}$ of the associated lifted $\mathbf{C}_{+}-$action $\hat{\varphi}_{\partial} \subset \widehat{G}_{1}$ on $X$ contains the union of the hypersurfaces $V_{z_{i}}=\left\{v=z_{i}\right\}$ for all the nonzero roots $z_{i}, i=2, \ldots, m$, of the polynomial $q$; if $q^{\prime}(0)=0$, then it contains $V_{0}$, too.

Proof. It is easy to check that $\partial$ resp. $\widehat{\partial}$ defined by the formulas (4) resp. (5) is, indeed, an LND of the algebra $\mathbf{C}^{[k+1]}$ resp. $\mathbf{C}^{[k+2]}=\mathbf{C}[\bar{x}, u, v]$. From (5) it follows that

$$
\widehat{\partial}(u v-p(\bar{x}))=v \cdot \frac{q(v)}{v} \delta(p(\bar{x}))-q(v) \delta(p(\bar{x}))=0,
$$

and so, $\hat{\partial}(J) \subset J$ where $J \subset \mathbf{C}^{[k+2]}=\mathbf{C}[\bar{x}, u, v]$ is the principal ideal generated by the polynomial $u v-p(\bar{x})$. Therefore, $\widehat{\partial}$ induces an LND of the quotient $\mathbf{C}[X]=$ $\mathbf{C}[\bar{x}, u, v] / J$. The other statements of the lemma can be verified without difficulty.

Example 5.1. In particular, putting in Lemma $5.2 \delta=\delta_{i}:=\frac{\partial}{\partial x_{i}} \in \operatorname{LND}\left(\mathbf{C}^{[k]}\right)$ we obtain $\partial=\partial_{i}=\partial_{i, q}:=q(v) \frac{\partial}{\partial x_{i}} \in \operatorname{LND}\left(\mathbf{C}^{[k+1]}\right)$ where LND $(A)$ denotes the set of all LND's of an algebra $A$. 
For the 'lifted' LND $\widehat{\partial} \in \operatorname{LND}\left(\mathbf{C}^{[k+2]}\right)$ we have

$$
\widehat{\partial}_{i}\left(x_{i}\right)=q(v), \quad \widehat{\partial}_{i}\left(x_{j}\right)=0 \text { if } j \neq i, \widehat{\partial}_{i}(v)=0, \widehat{\partial}_{i}(u)=v^{-1} q(v) \frac{\partial}{\partial x_{i}} p(\bar{x}) .
$$

It follows that $\widehat{\partial}_{i}^{2}\left(x_{i}\right)=0$ and $\widehat{\partial}_{i}^{n}(u)=v^{-1} q^{n}(v) \frac{\partial^{n}}{\partial x_{i}^{n}} p(\bar{x})$. The associated $\mathbf{C}_{+}-$action $\widehat{\varphi}_{i}:=\widehat{\varphi}_{\partial_{i}}$ on $\mathbf{C}^{[k+2]}$ is given as

$$
\begin{gathered}
\hat{\varphi}_{i}^{t}\left(x_{i}\right)=x_{i}+t q(v), \hat{\varphi}_{i}^{t}\left(x_{j}\right)=x_{j} \quad \text { if } j \neq i, \quad \hat{\varphi}_{i}^{t}(v)=v, \text { and } \\
\widehat{\varphi}_{i}^{t}(u)=u+\frac{1}{v} \sum_{n=1}^{\operatorname{deg} p} \frac{t^{n}}{n !} q^{n}(v) \frac{\partial^{n}}{\partial x_{i}^{n}} p(\bar{x}), \quad \text { i.e. } \\
\widehat{\varphi}_{i}^{t}\left(\bar{x}_{i}, u, v\right)=\left(\bar{x}+t q(v) \bar{e}_{i}, u+\frac{1}{v}\left[p\left(\bar{x}+t q(v) \bar{e}_{i}\right)-p(\bar{x})\right], v\right),
\end{gathered}
$$

where $\left(\bar{e}_{1}, \ldots, \bar{e}_{k}\right)$ is the standard basis of $\mathbf{C}^{k}$.

In the following lemma we keep all the notation from Lemma 5.2 and Example 5.1. We also denote by $\mathcal{O}_{\hat{\varphi}}(P)$ the orbit of a point $P \in X$ under a $\mathbf{C}_{+}-$action $\hat{\varphi}$ on $X$.

Lemma 5.3. Put $q(v)=v$. Then:

(a) For any point $P \in U_{0} \backslash \operatorname{sing} U_{0}$ there exists $i \in\{1, \ldots, k\}$ such that $\mathcal{O}_{\widehat{\varphi}_{i}}(P) \not \subset U_{0}$.

(b) For any point $P \in \operatorname{sing} U_{0} \backslash \operatorname{sing} X$ there exists $\delta \in \operatorname{LND}\left(\mathbf{C}^{[k]}\right)$ such that for the associated lifted $\mathbf{C}_{+}$-action $\widehat{\varphi}_{\partial}$ on $X$ one has $\mathcal{O}_{\widehat{\varphi}_{\partial}}(P) \not \subset \operatorname{sing} U_{0}$.

Proof. (a) As follows from Lemma 4.1(b), $P=\left(\bar{x}^{0}, 0, v^{0}\right) \notin \operatorname{sing} U_{0}$ iff $\operatorname{grad} \bar{x}^{0} p \neq 0$, i.e. $\frac{\partial p}{\partial x_{i}}\left(\bar{x}^{0}\right) \neq 0$ for some $i \in\{1, \ldots, k\}$. By $(6)$, the $u$-coordinate $u_{i}(t)$ of the point $\widehat{\varphi}_{i}^{t}\left(P_{0}\right) \in \mathcal{O}_{\widehat{\varphi}_{i}}(P)$ is a polynomial in $t$ with the non-zero linear term $\left(\frac{\partial p}{\partial x_{i}}\left(\bar{x}^{0}\right)\right) t$. Hence, $\mathcal{O}_{\widehat{\varphi}_{i}}(P) \not \subset U_{0}$, as claimed.

(b) Let $P=\left(\bar{x}^{0}, 0, v^{0}\right) \in \operatorname{sing} U_{0} \backslash \operatorname{sing} X$, that is, $\operatorname{grad}_{\bar{x}^{0}} p=0$, and $v^{0} \neq 0$. Fix a line $l$ through $\bar{x}^{0}$ in $\mathbf{C}^{k}$ such that the restriction $p \mid l$ is non-constant. Chose a new coordinate system in $\mathbf{C}^{k}$ with the first coordinate axis being parallel to $l$. Take $\delta=\delta_{1}=\frac{\partial}{\partial x_{1}} \in \operatorname{LND}\left(\mathbf{C}^{[k]}\right)$ with respect to the new coordinates, so that $\widehat{\varphi}_{\partial}=\widehat{\varphi}_{1}$. By (6), the projection $\bar{x}^{t}=\bar{x}^{0}+t v^{0} \bar{e}_{1}$ of the point $\hat{\varphi}_{1}^{t}(P) \in \mathcal{O}_{\widehat{\varphi}_{1}}(P)$ to $\mathbf{C}_{\bar{x}}^{k}$ runs over $l$. Since grad $p$ does not vanish identically on $l$, we obtain that $\mathcal{O}_{\widehat{\varphi}_{1}}(P) \not \subset \operatorname{sing} U_{0}$. The proof is completed.

Lemma 5.4. For any set of $m$ distinct points $P_{1}, \ldots, P_{m} \in X \backslash \operatorname{sing} X$ there exists an automorphism $\hat{\varphi} \in \widehat{G}_{1}$ of $X$ close enough to the identity such that $\hat{\varphi}\left(P_{i}\right) \notin U_{0}, i=$ $1, \ldots, m$.

Proof. Starting with $m=0$ assume, by induction, that the statement is true for any set of $m$ distinct points in $X \backslash \operatorname{sing} X$. Take an arbitrary set of $m+1$ distinct points $P_{1}, \ldots, P_{m+1} \in X \backslash \operatorname{sing} X$. By the inductive hypothesis, we may suppose that $P_{1}, \ldots, P_{m} \notin U_{0}$ and $P_{m+1} \in X \backslash \operatorname{sing} X$. After applying to $P=P_{m+1}$, if necessary, an automorphism $\widehat{\varphi}_{\delta}^{t} \in \widehat{G}_{1}$ of Lemma $5.3(\mathrm{~b})$ with $|t|$ small enough, we can achieve that $P_{m+1} \notin \operatorname{sing} U_{0}$, while still keeping $P_{1}, \ldots, P_{m} \notin U_{0}$. If it occurs that $P_{m+1} \in U_{0} \backslash \operatorname{sing} U_{0}$, then applying Lemma 5.3(a) to $P=P_{m+1}$ in the same way as above, we are done.

Notation. Denote by $G_{0}$ the subgroup of the group Aut $\mathbf{C}^{k}$ generated by all the $\mathbf{C}_{+}-$subgroups of Aut $\mathbf{C}^{k}$. 
The proof of the following lemma can be found in [J], Ka 2]; for the sake of completeness we reproduce it here.

Lemma 5.5. For any $k \geq 2$ and any $m \in \mathbf{N}$ the group $G_{0}$ acts $m$-transitively on $\mathrm{C}^{k}$.

Proof. The proof goes by induction on $k, k \geq 2$. Fix two arbitrary sets of $m$ distinct points $P_{1}, \ldots, P_{m}$ and $Q_{1}, \ldots, Q_{m}$ in $\mathrm{C}^{k}$ where $P_{i}=\left(\bar{x}^{(i)}, x_{n}^{(i)}\right)$ and $Q_{i}=\left(\bar{y}^{(i)}, y_{n}^{(i)}\right)$ for certain $\bar{x}^{(i)}, \bar{y}^{(i)} \in \mathbf{C}^{k-1}$ and $x_{n}^{(i)}, y_{n}^{(i)} \in \mathbf{C}, i=1, \ldots, m$. Choosing a generic coordinate system in $\mathbf{C}^{k}$ we may suppose that $\bar{x}^{(i)} \neq \bar{x}^{(j)}, x_{n}^{(i)} \neq x_{n}^{(j)}, \bar{y}^{(i)} \neq \bar{y}^{(j)}$ and $y_{n}^{(i)} \neq y_{n}^{(j)}$ for all $i \neq j$.

If $n>2$ then by the inductive hypothesis, we can find an automorphism $\alpha^{\prime} \in$ $G_{0}\left(\mathbf{C}^{k-1}\right)$ such that $\alpha^{\prime}\left(\bar{x}^{(i)}\right)=\bar{y}^{(i)}, i=1, \ldots, m$. After applying the automorphism $\alpha=\left(\alpha^{\prime}, \operatorname{id}_{\mathbf{C}}\right) \in G_{0}$ we may suppose that $\bar{y}^{(i)}=\bar{x}^{(i)}, i=1, \ldots, m$. Let $p \in \mathbf{C}^{[k-1]}$ be a polynomial such that $p\left(\bar{x}^{(i)}\right)=y_{n}^{(i)}-x_{n}^{(i)}, i=1, \ldots, m$. Consider the triangular $\mathbf{C}_{+}-$action $\beta^{t}$ on $\mathbf{C}^{k}$ given as $\beta^{t}:\left(\bar{x}, x_{n}\right) \longmapsto\left(\bar{x}, x_{n}+t p(\bar{x})\right)$. Then the automorphism $\beta:=\beta^{1} \in G_{0}$ sends $P_{i}$ to $Q_{i}, i=1, \ldots, m$. This provides the induction step.

For $k=2$ we start with the triangular automorphism $\alpha \in G_{0}, \alpha:\left(x_{1}, x_{2}\right) \longmapsto\left(x_{1}+\right.$ $\left.q\left(x_{2}\right), x_{2}\right)$ where $q \in \mathbf{C}[z]$ is a polynomial such that $q\left(x_{2}^{(i)}\right)=y_{1}^{(i)}-x_{1}^{(i)}, i=1, \ldots, m$, and then we apply $\beta \in G_{0}$, as above. This completes the proof.

Notation. For any set of $n+1$ distinct non-zero complex numbers $c_{0}, c_{1}, \ldots, c_{n} \in$ $\mathbf{C} \backslash\{0\}$ denote by $\operatorname{Stab}_{c_{1}, \ldots, c_{n}}\left(V_{c_{0}}\right)$ the subgroup of the group $\widehat{G}_{1}$ which consists of the automorphisms of $X$ leaving the hypersurface $V_{c_{0}}$ invariant and fixing each point of the hypersurfaces $V_{c_{i}}, i=1, \ldots, n$.

Lemma 5.6. (a) The group $\operatorname{Stab}_{c_{1}, \ldots, c_{n}}\left(V_{c_{0}}\right)$ acts $m$-transitively on $V_{c_{0}}$ for any $m \in$ N.

(b) For any set of $m$ distinct points $P_{1}, \ldots, P_{m} \in X \backslash \operatorname{sing} X$ there exists an automorphism $\widehat{\varphi} \in \widehat{G}$ of $X$ such that $\widehat{\varphi}\left(P_{i}\right) \in U_{1}, i=1, \ldots, m$.

Proof. (a) Fix two arbitrary sets of $m$ distinct points $P_{1}, \ldots, P_{m}$ and $Q_{1}, \ldots, Q_{m}$ in $V_{c_{0}}$ where $P_{i}=\left(\bar{x}^{(i)}, u^{(i)}, c_{0}\right), u^{(i)}=p\left(\bar{x}^{(i)}\right) / c_{0}$, and $Q_{i}=\left(\bar{y}^{(i)}, \tilde{u}^{(i)}, c_{0}\right), \tilde{u}^{(i)}=$ $p\left(\bar{y}^{(i)}\right) / c_{0}, i=1, \ldots, m$. By Lemma 5.5, there exists an automorphism $\alpha \in G_{0}$ such that $\alpha\left(\bar{x}^{(i)}\right)=\bar{y}^{(i)}, i=1, \ldots, m$.

Decompose $\alpha=\psi_{1}^{t_{1}} \circ \ldots \circ \psi_{l}^{t_{l}}$ into a product of elements of $\mathbf{C}_{+}$- subgroups of the group Aut $\mathbf{C}^{k}$, and let $\tilde{\delta}_{j} \in \operatorname{LND}\left(\mathbf{C}^{[k]}\right)$ be the infinitesimal generator of the subgroup $\left\{\psi_{j}^{t}\right\}_{t \in \mathbf{C}_{+}}, j=1, \ldots, l$.

Let also $q \in \mathbf{C}[z]$ be the degree $n$ polynomial with the roots $c_{1}, \ldots, c_{n}$ such that $q\left(c_{0}\right)=1$. Denote by $\tilde{\partial}_{j}=\partial_{\tilde{\delta}_{j}, q}$ the LND of the algebra $\mathbf{C}^{[k+1]}=\mathbf{C}[\bar{x}, v]$ defined as in (5) of Lemma 5.2(a), and by $\tilde{\varphi}_{j}$ the corresponding $\mathbf{C}_{+}-$action on $\mathbf{C}^{k+1}=\mathbf{C}_{\bar{x}}^{k} \times \mathbf{C}_{v}, j=$ $1, \ldots, l$. Then we have: $\tilde{\varphi}_{j}:\left(\bar{x}, c_{0}\right) \longmapsto\left(\psi_{j}(\bar{x}), c_{0}\right)$, and $\tilde{\varphi}_{j}$ fixes each point of the hyperplanes $D_{c_{i}}=\left\{v=c_{i}\right\}, i=1, \ldots, n$. The composition $\tilde{\alpha}=\tilde{\varphi}_{1}^{t_{1}} \circ \ldots \circ \tilde{\varphi}_{l}^{t_{l}}$ also fixes each point of the union $\bigcup_{i=1}^{n} D_{c_{i}}$, stabilizes the hyperplane $D_{c_{0}}$, and $\tilde{\alpha} \mid D_{c_{0}}=\alpha$, i.e. $\tilde{\alpha}\left(\bar{x}, c_{0}\right)=\left(\alpha(\bar{x}), c_{0}\right)$. Therefore, $\tilde{\alpha}\left(\bar{x}^{(i)}, c_{0}\right)=\left(\bar{y}^{(i)}, c_{0}\right), i=1, \ldots, m$.

By Lemma 5.2(b), the lift $\widehat{\alpha}=\widehat{\varphi}_{1}^{t_{1}} \circ \ldots \circ \widehat{\varphi}_{l}^{t_{l}} \in \widehat{G}_{1}$ fixes each point of the union $\bigcup_{i=1}^{n} V_{c_{i}}$ and stabilizes the hypersurface $V_{c_{0}}$, that is, $\widehat{\alpha} \in \operatorname{Stab}_{c_{1}, \ldots, c_{n}}\left(V_{c_{0}}\right)$. Moreover, since $\hat{\alpha}\left|V_{c_{0}}=\sigma_{1}^{-1} \tilde{\alpha} \sigma_{1}\right| V_{c_{0}}$ (see Lemma 5.1) we have $\widehat{\alpha}\left(P_{i}\right)=Q_{i}, i=1, \ldots, m$. This proves (a). 
(b) By Lemma 5.4, we may assume that $P_{i}=\left(\bar{x}^{(i)}, u^{(i)}, v^{(i)}\right) \in X \backslash V_{0}, i=1, \ldots, m$. Reordering, if necessary, the points $P_{i}$ we may also suppose that $v^{(1)}=\ldots=v^{\left(m^{\prime}\right)}=$ : $c_{0} \neq v^{(j)}, j=m^{\prime}+1, \ldots, m$. By (a), there exists an automorphism $\hat{\varphi} \in \widehat{G}_{1}$ such that $\widehat{\varphi}\left(P_{j}\right) \in V_{c_{0}} \cap U_{1}, j=1, \ldots, m^{\prime}$, and $\widehat{\varphi}\left(P_{j}\right)=P_{j}, j=m^{\prime}+1, \ldots, m$. Hence, proceeding by induction, we are done.

Proof of Theorem 5.1. Fix an arbitrary set of $m$ distinct points $P_{1}, \ldots, P_{m}$ in $X \backslash \operatorname{sing} X$ and another such set $Q_{1}, \ldots, Q_{m}$ in $U_{1}$. By Lemma 5.6(b), after applying, if necessary, to the points $P_{1}, \ldots, P_{m}$ an automorphism $\widehat{\varphi}_{1} \in \widehat{G}$ we may suppose that also $P_{1}, \ldots, P_{m} \in U_{1}$. Then, exchanging in Lemma 5.6(a) the roles of $U_{1}$ and $V_{1}$, by this lemma, we can find an automorphism $\widehat{\varphi}_{2} \in \widehat{G}_{2}$ such that $\widehat{\varphi}_{2}\left(P_{i}\right)=Q_{i}, i=1, \ldots, m$. The proof is completed.

\section{Examples of acyclic surfaces in $\mathbf{C}^{3}$ and of smooth contractible 4 -folds $u v=p(x, y, z)$ in $\mathbf{C}^{5}$}

In the examples of smooth acyclic surfaces in $\mathbf{C}^{3}$ with big fundamental groups (see Example 6.1 below) we use the following simple lemma (cf. [Za 2, Lemma 7.2]).

Lemma 6.1. Let $Y$ be a connected simply connected complex manifold, $F$ be a smooth irreducible hypersurface in $Y$, and $p: X \rightarrow Y$ be a branched cyclic covering over $Y$ ramified to order $s$ on $F$. Denote $G_{X}=\pi_{1}\left(X^{*}\right)$ resp. $G_{Y}=\pi_{1}\left(Y^{*}\right)$, where $X^{*}:=$ $X \backslash p^{-1}(F)$ and $Y^{*}:=Y \backslash F$. Identifying $G_{X}$ with the index s subgroup $p_{*}\left(G_{X}\right) \subset G_{Y}$ we denote by $\beta_{F}$ a vanishing loop of $F$ in $Y$ and by $<<\left(\beta_{F}\right)^{s}>>$ the normal closure in $G_{Y}$ of the cyclic subgroup generated by $\left(\beta_{F}\right)^{s}$. Then $\pi_{1}(X) \simeq G_{X} /<<\left(\beta_{F}\right)^{s}>>$, and it is an index s subgroup of the group $G_{Y} /<<\left(\beta_{F}\right)^{s}>>$ with a cyclic quotient.

Proof. We have the following commutative diagram:

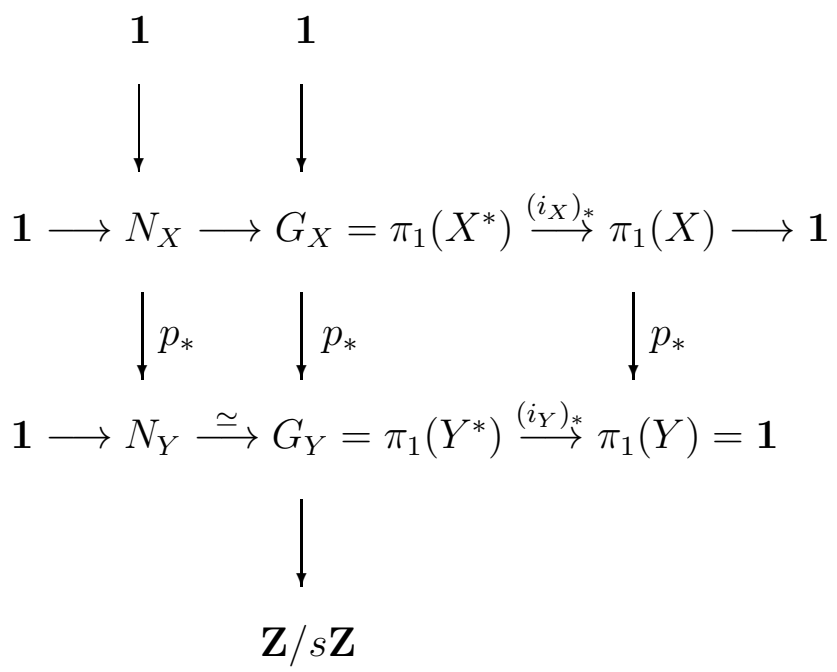

where $i_{X}: X^{*} \hookrightarrow X$ resp. $\quad i_{Y}: Y^{*} \hookrightarrow Y$ denotes the identical embedding and $N_{X}:=\operatorname{ker}\left(i_{X}\right)_{*} \operatorname{resp} . \quad N_{Y}:=\operatorname{ker}\left(i_{Y}\right)_{*}$. Thus, $\pi_{1}(X) \simeq G_{X} / N_{X}, \quad \mathbf{1}=\pi_{1}(Y) \simeq G_{Y} / N_{Y}$ where $N_{X}=<<\alpha_{F}>>$ and $N_{Y}=<<\beta_{F}>>$ with $\alpha_{F} \in N_{X}$ resp. $\beta_{F} \in N_{Y}$ being a vanishing loop of $p^{-1}(F) \simeq F$ in $X$ resp. of $F$ in $Y$; see e.g. [Za 2, (2.3.a)]. Moreover, identifying $G_{X}$ with the subgroup $p_{*}\left(G_{X}\right) \subset G_{Y}$ we may assume that $\alpha_{F}=\left(\beta_{F}\right)^{s}$. 
Since the quotient $G_{Y} / G_{X} \simeq \mathbf{Z} / s \mathbf{Z}$ is abelian we have $K_{Y}:=\left[G_{Y}, G_{Y}\right] \subset G_{X}$. Since $G_{Y}=N_{Y}=<<\beta_{F}>>$ the abelianization $\left(G_{Y}\right)_{\mathrm{ab}}:=G_{Y} / K_{Y}$ is a cyclic group generated by the class $K_{Y} \beta_{F}$. Hence, any element $g \in G_{Y}$ can be written as $g=g^{\prime}\left(\beta_{F}\right)^{t}$ where $g^{\prime} \in K_{Y} \subset G_{X}$ and $t \in \mathbf{Z}$. Thus, we have $g\left(\beta_{F}\right)^{s} g^{-1}=g^{\prime}\left(\beta_{F}\right)^{s} g^{\prime-1}$ for any $g \in G$. Therefore, the normal closure $N_{X}$ of the cyclic subgroup $\left\langle\left(\beta_{F}\right)^{s}=\alpha_{F}\right\rangle$ in the group $G_{X}$ coincides with its normal closure in the bigger group $G_{Y}$, i.e. $N_{X} \subset G_{Y}$ is a normal subgroup and it coincides with the subgroup $<<\left(\beta_{F}\right)^{s}>>$. Hence, we have $\pi_{1}(X) \simeq G_{X} / N_{X} \simeq G_{X} /<<\left(\beta_{F}\right)^{s}>>$, as required.

The chain of normal subgroups $N_{X} \subset G_{X} \subset G_{Y}$ yields the short exact sequence

$$
\mathbf{1} \longrightarrow \pi_{1}(X) \simeq G_{X} / N_{X} \longrightarrow G_{Y} / N_{X} \longrightarrow G_{Y} / G_{X} \simeq \mathbf{Z} / n \mathbf{Z} \longrightarrow \mathbf{1}
$$

and the last assertion of the lemma follows.

Example 6.1. Let $X_{k, l, s, m}=p_{k, l, s, m}^{-1}(0) \subset \mathbf{C}^{3}$ be the surface defined by the polynomial

$$
p_{k, l, s, m}=\frac{\left(x z^{m}+1\right)^{k}-\left(y z^{m}+1\right)^{l}-z^{s}}{z^{m}} \in \mathbf{C}[x, y, z]
$$

where $0 \leq m \leq s$. It is smooth if $m>0$, and it has at most one singular point $P_{0}=(1,1,0)$ if $m=0$. For $\operatorname{gcd}(k, l)=\operatorname{gcd}(k, s)=\operatorname{gcd}(l, s)=1$ and $m=s$ the surface $Y_{k, l, s}:=X_{k, l, s, s}$ is acyclic. Indeed, it can be presented as a cyclic $\mathbf{C}^{*}$ - covering over the contractible tom Dieck-Petrie surface $X_{k, l}=X_{k, l, 1,1} \subset \mathbf{C}^{3}$ (see Example 3.1) branched to order $s$ along the line $L_{k, l}:=X_{k, l} \cap\{z=0\} \simeq \mathbf{C}$ in $X_{k, l}$, and the acyclicity follows as in the proof of Theorem $\mathrm{A}$ in [Ka 1] (see also [Za 2, §5]).

However, in general the acyclic surface $Y_{k, l, s}$ is not contractible and possesses quite a big fundamental group. Indeed, let $\sigma: \mathbf{C}^{3} \rightarrow \mathbf{C}^{3}, \sigma(x, y, z)=(x z, y z, z)$, be the affine modification of $\mathbf{C}^{3}$ along the plane $z=0$ with center at the origin (see Example 1.3). Then the restriction $\sigma \mid X_{k, l, s, m}: X_{k, l, s, m} \rightarrow X_{k, l, s, m-1}$ is the affine modification of $X_{k, l, s, m-1}$ along the line $D:=X_{k, l, s, m-1} \cap\{z=0\}$ with center at the origin (see Example 2.1).

Furthermore, the surface $X_{k, l, s, 1}$ coincides with the pseudoaffine modification of the smooth quasiprojective surface $X_{k, l, s, 0}^{\prime}:=X_{k, l, s, 0} \backslash\left\{P_{0}\right\}$ along the smooth curve $D^{*}:=$ $D \backslash\left\{P_{0}\right\}$ with center $\overline{0} \in D^{*}$ (see Definition 3.1).

By Corollary 3.1, the surface $Y_{k, l, s}=X_{k, l, s, s}$ being acyclic also the surfaces $X_{k, l, s, m}$ are acyclic for all $m=1, \ldots, s$. The repeated application of Lemma 3.4 in [Ka 1] (or of Proposition 3.1 above) yields the isomorphisms

$$
\pi_{1}\left(Y_{k, l, s}\right)=\pi_{1}\left(X_{k, l, s, s}\right) \simeq \pi_{1}\left(X_{k, l, s, s-1}\right) \simeq \ldots \simeq \pi_{1}\left(X_{k, l, s, 1}\right) \simeq \pi_{1}\left(X_{k, l, s, 0}^{\prime}\right) .
$$

The surface $X_{k, l, s, 0} \simeq X_{k, l, s}:=\left\{x^{k}-y^{l}-z^{s}=0\right\} \subset \mathbf{C}^{3}$ is homotopy equivalent to the cone over the Pham-Brieskorn 3-manifold $M_{k, l, s}:=X_{k, l, s} \cap S^{5}$, that is, over the link of the surface singularity of $X_{k, l, s}$ in the sphere $S^{5}$. In turn, $X_{k, l, s, 0}^{\prime} \simeq X_{k, l, s} \backslash\{\overline{0}\}$ is homotopy equivalent to the link $M_{k, l, s}$, and thus $\pi_{1}\left(Y_{k, l, s}\right) \simeq \pi_{1}\left(M_{k, l, s}\right)$; denote the latter group by $G_{k, l, s}^{\prime}$.

The structure of the group $G_{k, l, s}^{\prime}$ is well known Mil 2]. It is finite iff $1 / k+1 / l+$ $1 / s>1$, infinite nilpotent iff $1 / k+1 / l+1 / s=1$. If $1 / k+1 / l+1 / s \neq 1$, then $G_{k, l, s}^{\prime}=\left[G_{k, l, s}, G_{k, l, s}\right]$ where

$$
G_{k, l, s}:=<\gamma_{1}, \gamma_{2}, \gamma_{3} \mid \gamma_{1}^{k}=\gamma_{2}^{l}=\gamma_{3}^{s}=\gamma_{1} \gamma_{2} \gamma_{3}>
$$


is a central extension of the Schwarz triangular group

$$
T_{k, l, s}:=<b_{1}, b_{2}, b_{3} \mid b_{1}^{2}=b_{2}^{2}=b_{3}^{2}=1,\left(b_{1} b_{2}\right)^{k}=\left(b_{2} b_{3}\right)^{l}=\left(b_{3} b_{1}\right)^{s}=1>.
$$

Note that for $1 / k+1 / l+1 / s<1$ the Schwarz triangular group $T_{k, l, s}$ contains a free subgroup with two generators. Therefore, the group $G_{k, l, s}^{\prime}$ also contains such a subgroup; in particular, it is not solvable. It is known [Bri] that the Pham-Brieskorn manifold $M_{k, l, s}$ is a homology 3-sphere iff $\operatorname{gcd}(k, l)=\operatorname{gcd}(k, s)=\operatorname{gcd}(l, s)=1$. Under this condition the group $G_{k, l, s}^{\prime}$ is perfect, i.e. coincides with its commutator subgroup; indeed, its abelianization $H_{1}\left(Y_{k, l, s} ; \mathbf{Z}\right)$ is trivial. Notice that the condition of relative primeness never holds in the Euclidean case $1 / k+1 / l+1 / s=1$; in the spherical one $1 / k+1 / l+1 / s>$ 1 it holds only for the Kleinian icosahedral triple $(k, l, s)=(2,3,5)$.

The isomorphism $X_{k, l}^{*}:=X_{k, l} \backslash L_{k, l} \simeq \mathbf{C}^{2} \backslash \Gamma_{k, l}$ where $\Gamma_{k, l}:=\left\{x^{k}-y^{l}=0\right\} \subset \mathbf{C}^{2}$ tDP provides the presentation

$$
B_{k, l}:=\pi_{1}\left(X_{k, l}^{*}\right)=<a, b \mid a^{k}=b^{l}>
$$

(see e.g. [Di 2]). Since $Y_{k, l, s}^{*}:=Y_{k, l, s} \backslash\{z=0\} \rightarrow X_{k, l}^{*}, \quad(x, y, z) \longmapsto\left(x, y, z^{s}\right)$, is a non-ramified cyclic covering, the group $\pi_{1}\left(Y_{k, l, s}^{*}\right)$ is isomorphic to an index $s$ subgroup, say, $\tilde{C}_{k, l, s}$ of the group $B_{k, l}$ with the cyclic quotient $B_{k, l} / \tilde{C}_{k, l, s} \simeq \mathbf{Z} / s \mathbf{Z}$. We have $\operatorname{ker}\left(i_{*}: \pi_{1}\left(Y_{k, l, s}^{*}\right) \rightarrow \pi_{1}\left(Y_{k, l, s}\right)\right)=<<\alpha^{s}>>$ where $\alpha \in B_{k, l}$ is a vanishing loop of the line $L_{k, l} \subset X_{k, l}$ [Za 2, (2.3.a)]. It can be shown that $\alpha=a^{q} b^{p} \in B_{k, l}$ where $p, q \in \mathbf{Z}$ are such that $k p+l q=1$.

Therefore, for $\operatorname{gcd}(k, l)=\operatorname{gcd}(k, s)=\operatorname{gcd}(l, s)=1$ the group $G_{k, l, s}^{\prime} \simeq \pi_{1}\left(Y_{k, l, s}\right)$ is isomorphic to an index $s$ subgroup $C_{k, l, s}$ of the quotient

$$
B_{k, l, s}:=B_{k, l} /<<\alpha^{s}>>=<a, b \mid a^{k}=b^{l},\left(a^{q} b^{p}\right)^{s}=1>
$$

(see Lemma 6.1 above). In particular, for $k=2, l=3$ in view of the isomorphism $X_{2,3}^{*} \simeq \mathbf{C}^{2} \backslash \Gamma_{2,3}$ we have that $B_{2,3}=B_{3}$ is the 3-braid group with the generators $\sigma_{1}, \sigma_{2} \in B_{3}$ being vanishing loops of $L_{2,3}$ in $X_{2,3}, a=\sigma_{1} \sigma_{2} \sigma_{1}, b=\sigma_{1} \sigma_{2}$. Therefore, $G_{2,3, s}^{\prime}$ is isomorphic to an index $s$ subgroup $C_{2,3, s}$ of the group

$$
B_{2,3, s}=B_{3} /<<\sigma_{1}^{s}>>=<\sigma_{1}, \sigma_{2} \mid \sigma_{1} \sigma_{2} \sigma_{1}=\sigma_{2} \sigma_{1} \sigma_{2}, \sigma_{1}^{s}=\sigma_{2}^{s}=1>
$$

which consists of the elements of algebraic length 14 divisible by $s$.

Example 6.2. Consider further the 4 -fold $X=X^{k, l, s, m}$ in $\mathbf{C}^{5}$ given by the equation $u v-p_{k, l, s, m}(x, y, z)=0$. By Corollary 4.1, the surface $X_{k, l, s, m}$ being acyclic implies that the hypersurface $X \subset \mathbf{C}^{5}$ is diffeomorphic to the Euclidean space $\mathbf{R}^{8}$. But in general, as we have seen above, the surface $X_{0}=X_{k, l, s, m} \subset \mathbf{C}^{3}$ is not contractible and possesses quite a big fundamental group. This shows that Proposition 4.2 cannot be extended to $k=3$.

Next we give an example of a polynomial $p=p_{2,3}^{0} \in \mathbf{C}^{[3]}$ with a smooth acyclic (even contractible) zero fibre $F_{0}=p^{-1}(0)$ and non-acyclic generic fibres $F_{c}=p^{-1}(c)$. In fact, the surface $F_{0}$ in this example is isomorphic to the tom Dieck-Petrie surface $X_{2,3}$

\footnotetext{
${ }^{14}$ Recall that the algebraic length of an element $\prod_{n=1}^{m} \sigma_{i_{n}}^{a_{n}}$ is the integer $\sum_{n=1}^{m} a_{n}$.
} 
(see Example 6.1 above), but the embedding $X_{2,3} \stackrel{\simeq}{\longrightarrow} F_{0} \subset \mathbf{C}^{3}$ is not equivalent to the standard one up to the action on $\mathbf{C}^{3}$ of the automorphism group Aut $\mathbf{C}^{3}$. This provides also examples of non-equivalent embeddings of an exotic $\mathbf{C}^{n}$ into $\mathbf{C}^{n+1}$.

Example 6.3. Consider the affine modification $\sigma=\sigma_{\overline{0}, H}: \mathbf{C}^{4} \rightarrow \mathbf{C}^{4}, \sigma:(x, y, z, t) \longmapsto$ $(x, x y, x z, x t)$, of $\mathbf{C}^{4}$ along the hyperplane $H_{0}=\{x=0\}$ with center at the origin (cf. Example 1.3). Consider also the Russell cubic $X \subset \mathrm{C}^{4}$ with the equation

$$
-x+x^{2} y+(z+1)^{2}-(t+1)^{3}=0
$$

(cf. Example 1.5). By Corollary 2.1, the restriction of $\sigma$ to the strict transform $X^{\prime}$ of $X$ yields the affine modification $\sigma_{\overline{0}, B}: X^{\prime}=\Sigma_{\overline{0}, B}(X) \rightarrow X$ of $X$ along the book-surface $B=H_{0} \cap X \simeq \Gamma_{2,3} \times \mathbf{C}$ with center $\overline{0} \in B \backslash \operatorname{sing} B$. The hypersurface $X^{\prime} \subset \mathbf{C}^{4}$ is given by the equation

$$
-1+x^{2} y+\frac{(x z+1)^{2}-(x t+1)^{3}}{x}=0
$$

(cf. Example 2.1). The isomorphism $\mathbf{C}^{3} \simeq X^{\prime}$ as in Example 1.5 provides an embedding of $\mathbf{C}^{3}$ into $\mathbf{C}^{4}$. A direct computation shows that this embedding is rectifiable. 15

The hyperplane section $X_{2,3}^{0}:=X^{\prime} \cap D_{0}$ where $D_{0}=\{y=0\}$, is isomorphic to the tom Dieck-Petrie surface $X_{2,3}$ (see Example 6.1). But the embedding $X_{2,3} \stackrel{\simeq}{\longrightarrow}$ $X_{2,3}^{0} \hookrightarrow X^{\prime} \simeq \mathbf{C}^{3}$ of the tom Dieck-Petrie surface into $\mathbf{C}^{3}$ is not equivalent to the standard one $X_{2,3} \hookrightarrow D_{0}=\mathbf{C}^{3}$. Indeed, the latter one is defined by the polynomial $p_{2,3}=\frac{(x z+1)^{2}-(x t+1)^{3}}{x}-1 \in \mathbf{C}^{[3]}$ with all the fibres being contractible surfaces; see e.g. [Za 2, Example 6.1]. On the other hand, it is easily seen that for a generic $c \in \mathbf{C}$ the fibre $F_{c}=p^{-1}(c)$ of the regular function $p=p_{2,3}^{0}:=y \mid X^{\prime} \in \mathbf{C}\left[X^{\prime}\right] \simeq \mathbf{C}^{[3]}$ which defines the surface $X_{2,3}^{0}$ in $X^{\prime}$ has the Euler characteristic $e\left(F_{c}\right)=5$ (hint: use the fibration $F_{c} \rightarrow \mathbf{C}$ defined by the restriction $\left.x \mid F_{c}\right)$. In particular, the surfaces $F_{c}$ for a generic $c \in \mathbf{C}$ are not acyclic.

Consider further the exotic product-structure $X_{2,3, n}:=X_{2,3} \times \mathbf{C}^{n-2}$ on $\mathbf{C}^{n}, n \geq 3$ (see [Za 2, §4]). By the similar arguments as above, two realizations $X_{2,3, n}:=X_{2,3} \times$ $\mathbf{C}^{n-2} \hookrightarrow \mathbf{C}^{n+1}=\mathbf{C}^{3} \times \mathbf{C}^{n-2}$ and $X_{2,3, n}^{0}:=X_{2,3}^{0} \times \mathbf{C}^{n-2} \hookrightarrow \mathbf{C}^{n+1}$ of this exotic $\mathbf{C}^{n}$ as a hypersurface in $\mathbf{C}^{n+1}$ are not equivalent modulo the action on $\mathbf{C}^{n+1}$ of the automorphism group Aut $\mathbf{C}^{n+1}$.

\section{Remarks}

6.1. By Corollary 4.1, the zero fibres of the polynomials $u v-p_{2,3}(x, y, z) \in \mathbf{C}^{[5]}$ and $u v-$ $p_{2,3}^{0}(x, y, z) \in \mathbf{C}^{[5]}$, as well as the generic fibres of the first one, are smooth contractible hypersurfaces in $\mathbf{C}^{5}$, whereas the generic fibres of the second one have Euler characteristic 5 (see Lemma 4.1(a)).

6.2. More generally, we have presented above a collection of examples of smooth acyclic hypersurfaces in $\mathbf{C}^{n}$ (see also [ $\left.\mathrm{Za} 2\right]$ ). Most of them are not rectifiable; actually, their defining polynomials have a fibre non-isomorphic to $\mathbf{C}^{n-1}$. By Corollary 3.1 (see also

\footnotetext{
${ }^{15}$ It is rectifiable e.g. via the composition $\gamma \circ \beta \circ \alpha$ of the triangular automorphisms $\alpha:(x, y, z, t) \longmapsto(x, y, u, t)$ where $u=z+f(x, t)=z-x t^{2}(x t+3) / 2$,

$\beta:(x, y, u, t) \longmapsto(x, v, u, t) \quad$ where $\quad v=y+g(x, u, t)=y+u t^{2}(x t+3)+x t^{4}(x t+3)^{2} / 4$ and

$\gamma:(x, v, u, t) \longmapsto(x, v, u, w)$ where $w=-3 t+h(x, u, v)=-3 t+x^{2} v+x u^{2}+2 u-1$.
} 
Remark 3.1), performing the affine modification of $\mathbf{C}^{n}$ along such a hypersurface $D=$ $p^{*}(0), p \in \mathbf{C}^{[n]}$, with a smooth reduced acyclic center $C \subset D$ leads to a smooth contractible affine $n$-fold $X$. The question arises when $X$ itself is isomorphic to the affine space $\mathbf{C}^{n}$ (cf. Question A in sect. 7 below).

Suppose that this is the case, and that, moreover, $C \simeq \mathbf{C}^{k}$. Then the exceptional divisor $E=\sigma_{C}^{-1}(C)=q^{*}(0) \subset X$ where $\sigma_{C}: X \rightarrow \mathbf{C}^{n}$ is the blowup morphism and $q:=p \circ \sigma_{C} \in \mathbf{C}[X] \simeq \mathbf{C}^{[n]}$, would be isomorphic to $\mathbf{C}^{n-1}$. But $q$ having a fibre nonisomorphic to $\mathbf{C}^{n-1}$, the hypersurface $E \simeq \mathbf{C}^{n-1}$ in $X \simeq \mathbf{C}^{n}$ cannot be rectifiable. Thus, this would answer in negative to the Abhyankar-Sathaye Embedding Problem (cf. Remark 4.3) 16 .

\section{On the theorems of Sathaye and Wright}

We propose the following

\section{Questions}

A) When an affine modification $X=\Sigma_{I, f}\left(\mathbf{C}^{n}\right)$ of $\mathbf{C}^{n}$ is isomorphic to $\mathbf{C}^{n}$ ?

B) Let $X=\Sigma_{I, f}\left(\mathbf{C}^{n}\right)$ be an affine modification of $\mathbf{C}^{n}$ canonically embedded into $\mathbf{C}^{n+k}$ (see Definition 1.2). If $X \simeq \mathbf{C}^{n}$, is it necessarily rectifiable in $\mathbf{C}^{n+k}$ ?

C) Which embeddings $\mathbf{C}^{n} \hookrightarrow \mathbf{C}^{n+k}$ appear in this way?

Question B is a specialization of the Abhyankar-Sathaye Embedding Problem mentioned in the Introduction. This problem is known to be answered in affirmative for $k \geq n+2$ (the Jelonek-Kaliman-Nori-Srinivas Theorem [J], Ka 2, Sr]) and for $\mathrm{n}=\mathrm{k}=1$ (the Abhyankar-Moh and Suzuki Embedding Theorem [AM, SuZ]). In a special case when $n=2$ and $k=1$ the positive answer to Question $\mathbf{B}$ is provided by a theorem of A. Sathaye [Sat], generalized by D. Wright 18 Wr 2] as follows.

\section{Theorem 7.1 (Sathaye-Wright).}

Let $X=X_{n, f, g}$ be a surface in $\mathbf{C}^{3}$ given by the equation $f(x, y) z^{n}+g(x, y)=0$ where $f, g \in \mathbf{C}[x, y], n \in \mathbf{N}$. Suppose that $X \simeq \mathbf{C}^{2}$. Then $X$ is rectifiable, i.e. there exists an automorphism $\alpha \in$ Aut $\mathbf{C}^{2}$ which transforms $X$ into a coordinate plane.

We give below a new proof of Theorem 7.1, as well as some generalization. The proof is easy if one of the polynomials $f, g$ is constant; in the sequel we do not consider this possibility. Observe that for $n=1$ the surface $X=X_{1, f, g}$ as in Theorem 7.1 is the affine modification $\Sigma_{I, f}\left(\mathbf{C}^{2}\right)$ of $\mathbf{C}^{2}$ along the divisor $D_{f}=f^{*}(0)$ with center $I=(f, g)$ (see Example 1.4) whereas the surface $X_{n, f, g}$ can be presented as a cyclic covering of $X_{1, f, g}$ ramified to order $n$ on $D_{g}=g^{*}(0)$. So, in the proof we use affine modifications. Theorem 7.2. Let $X=X_{n, f, g}$ be an irreducible smooth surface in $\mathbf{C}^{3}$ given by the equation $f(x, y) z^{n}+g(x, y)=0$ where $f, g \in \mathbf{C}[x, y], n \in \mathbf{N}$. The following conditions (i) - (iv) are equivalent 19 :

\footnotetext{
${ }^{16}$ Recall that the varieties discussed in Remark 4.3 arise as affine modifications of the affine space $\mathbf{C}^{n}$ along a hyperplane with non-linear acyclic centers. Whereas here we consider, in particular, affine modifications of $\mathbf{C}^{n}$ along acyclic hypersurfaces with center at a linear subspace.

${ }^{17}$ which corresponds to the case $n=1$ in Theorem 7.1 below.

${ }^{18}$ See also $\mathrm{Ru} 2$, RuSat for some generalizations.

${ }^{19}$ Hereafter $e(X)$ denotes the Euler characteristic of $X$.
} 
(i) $e(X)=1$ and $H_{1}(X ; \mathbf{Z})=0$.

(ii) $X$ is acyclic, i.e. $\tilde{H}_{*}(X ; \mathbf{Z})=0$.

(iii) $X \simeq \mathbf{C}^{2}$.

(iv) $X$ is rectifiable.

If $n>1$, then the above conditions are equivalent to the following one:

(v) The pair $(f, g)$ is rectifiable in the following sense: there exists an automorphisms $\alpha \in$ Aut $\mathbf{C}^{[2]}$ such that $(\alpha(f), \alpha(g))=(p(x), y)$.

The implications $(v) \Longrightarrow(i v) \Longrightarrow(i i i) \Longrightarrow(i i) \Longrightarrow(i)$ are easy; in the sequel we only prove $(i) \Longrightarrow(i v)$ in the case $n=1$ and $(i) \Longrightarrow(v)$ in the case $n>1$.

\section{Remarks}

7.1. There are examples of acyclic or even contractible smooth algebraic surfaces in $\mathbf{C}^{3}$ non-isomorphic to $\mathbf{C}^{2}$, see e.g. [tDP, KaML 2] and Example 6.1 below. Moreover, any smooth contractible affine algebraic surface of logarithmic Kodaira dimension 1 admits such an embedding into $\mathbf{C}^{3}$ KaML 2]. However, Theorem 7.2 shows that the image of this embedding cannot be given by a 'binomial' equation $f(x, y) z^{n}+g(x, y)=0$.

7.2. As a corollary we obtain that if the zero fibre of the polynomial $f(x, y) z^{n}+g(x, y)$ is acyclic (resp. isomorphic to $\mathbf{C}^{2}$ ) then so is every fibre. Notice that in general, a polynomial $p \in \mathbf{C}^{[3]}$ with a smooth acyclic, or even contractible, zero fibre may have non-acyclic generic fibres; see Example 6.3 above.

7.3. The next simple observation will be useful in what follows. Let $X_{1}=X_{1, f, g}$ be a surface as in Theorem 7.2 with $n=1$. Since $X_{1}$ is supposed being irreducible, the divisors $D_{f}$ and $D_{g}$ have no irreducible component in common. Thus, the center $D_{f} \cdot D_{g}$ of the blow up $\sigma_{I}: X_{1} \rightarrow \mathbf{C}^{2}, \sigma_{I}(x, y, z)=(x, y)$, is supported by the finite set supp $D_{f} \cap \operatorname{supp} D_{g}$ (see Example 1.4). The exceptional curve $E^{\prime} \subset X_{1}, E^{\prime}=$ $\{f(x, y)=g(x, y)=0\}$, is isomorphic to the product $\mathbf{C} \times\left(\operatorname{supp} D_{f} \cap \operatorname{supp} D_{g}\right)$, and hence, it consists of $\kappa$ vertical lines in $X_{1}$ where $\kappa:=\operatorname{card}\left(\operatorname{supp} D_{f} \cap \operatorname{supp} D_{g}\right)$.

The proof of Theorem 7.2 is based on the following Lemmas 7.1 - 7.6.

Lemma 7.1. Let $X$ be a smooth irreducible affine surface. Then

(a) The following conditions $(i)$ and $(i i)$ (resp. $\left(i^{\prime}\right)$ and $\left(i i^{\prime}\right)$ ) are equivalent:

(i) $e(X)=1$ and $H_{1}(X ; \mathbf{Z})=0$ resp. $\left(i^{\prime}\right) e(X)=1$ and $b_{1}(X)=0$;

(ii) $X$ is acyclic, i.e. $\tilde{H}_{*}(X ; \mathbf{Z})=0$, resp. $\left(i i^{\prime}\right) X$ is $\mathbf{Q}-$ acyclic, i.e. $\tilde{H}_{*}(X ; \mathbf{Q})=$ 0 .

(b) If ( $i$ ) holds then the algebra $A=\mathbf{C}[X]$ is UFD.

Proof. (a) The implications $(i i) \Longrightarrow(i)$ resp. $\left(i i^{\prime}\right) \Longrightarrow\left(i^{\prime}\right)$ are straightforward. To prove the converse ones, notice that by the Lefchetz Hyperplane Section Theorem Mil 1, Thm. 7.2], $X$ has homotopy type of a finite cell complex of real dimension at most two. Hence, $H_{3}(X ; \mathbf{Z})=H_{4}(X ; \mathbf{Z})=0$ and $H_{2}(X ; \mathbf{Z})$ is a free abelian group. Therefore, $e(X)=1-b_{1}(X)+b_{2}(X)$, and so, if $e(X)=1$ and $b_{1}(X)=0$, then $b_{2}(X)=0$, and moreover, $H_{2}(X ; \mathbf{Z})=0$. Thus, $(i)$ implies that $\tilde{H}_{*}(X ; \mathbf{Z})=0$, i.e. $X$ is acyclic; in turn, $\left(i^{\prime}\right)$ implies that $\tilde{H}_{*}(X ; \mathbf{Q})=0$, i.e. $X$ is $\mathbf{Q}-$ acyclic. This proves (a).

In view of (a), (b) follows from [Fuj, (1.17)-(1.20)] (see also [Ka 1, Prop. 3.2]).

Lemma 7.2. Let $f, g \in \mathbf{C}^{[2]} \backslash \mathbf{C}$ be two non-constant polynomials without common factor 20. Then the surface $X_{n}=X_{n, f, g}=\left\{f z^{n}+g=0\right\} \subset \mathbf{C}^{3}$ is smooth iff the

\footnotetext{
${ }^{20}$ For instance, this is so if the surface $X_{n}=X_{n, f, g}$ is irreducible.
} 
following two conditions are fulfilled:

(i) For any point $P_{0} \in \operatorname{supp} D_{f} \cap \operatorname{supp} D_{g}$ the divisor $D_{g}$ is non-singular and reduced at $P_{0}$. If so is the divisor $D_{f}$ at $P_{0}$ too, then $D_{f}$ and $D_{g}$ are transversal at $P_{0}$.

(ii) If $n>1$, then $D_{g}$ is a smooth reduced divisor.

Proof. The statement easily follows from the equality

$$
\operatorname{grad}\left(f z^{n}-g\right)=\left(z^{n} \operatorname{grad} f-\operatorname{grad} g, n z^{n-1} f\right) .
$$

Lemma 7.3. Let $X$ be a connected complex manifold, and let $D_{1}, \ldots, D_{s}$ be reduced irreducible principal divisors in $X$. Set $D=\bigcup_{i=1}^{s} D_{i}$ and $X^{*}=X \backslash D$. Then there is an exact sequence

$$
\mathbf{0} \longrightarrow \mathbf{Z}^{s} \stackrel{\mu}{\longrightarrow} H_{1}\left(X^{*} ; \mathbf{Z}\right) \stackrel{i_{*}^{\prime}}{\longrightarrow} H_{1}(X ; \mathbf{Z}) \longrightarrow \mathbf{0},
$$

where $i: X^{*} \hookrightarrow X$ is the identical embedding, and $\mu$ sends the standard basis vectors $\left(e_{1}, \ldots, e_{s}\right)$ of the lattice $\mathbf{Z}^{s}$ into the the vanishing loop classes $\alpha_{1}, \ldots, \alpha_{s}$ of $D_{1}, \ldots, D_{s}$, respectively. Moreover, this sequence splits.

Proof. We have the following exact sequence of fundamental groups:

$$
\mathbf{1} \longrightarrow<<\alpha_{1}, \ldots, \alpha_{s}>>\longrightarrow \pi_{1}\left(X^{*}\right) \stackrel{i_{*}^{\prime}}{\longrightarrow} \pi_{1}(X) \longrightarrow \mathbf{1}
$$

where $<<\alpha_{1}, \ldots, \alpha_{s}>>$ denotes the normal subgroup of the group $\pi_{1}\left(X^{*}\right)$ generated by these vanishing loops (see e.g. [Za 2, (2.3.a)]). Passing to the abelianizations shows that the above homology sequence is exact besides, possibly, at the second term. The divisor $D_{i}^{\prime}$ being principal we have $D_{i}^{\prime}=g_{i}^{*}(0)$ where $g_{i}$ is a holomorphic function on $X, i=1, \ldots, s$. The morphism $\varphi=\left(g_{1}, \ldots, g_{s}\right): X_{1}^{*} \rightarrow\left(\mathbf{C}^{*}\right)^{s}$ yields a surjection

$$
\varphi_{*}: H_{1}\left(X^{*} ; \mathbf{Z}\right) \longrightarrow \mathbf{Z}^{s}, \varphi_{*}\left(\alpha\left(D_{i}^{\prime}\right)\right)=e_{i}, i=1, \ldots, s .
$$

This provides the exactness at the second term, the splitting $H_{1}\left(X^{*} ; \mathbf{Z}\right) \simeq H \oplus$ ker $\varphi_{*}$ where $H=<\alpha_{1}, \ldots, \alpha_{s}>$, and an isomorphism $H_{1}(X ; \mathbf{Z}) \simeq \operatorname{ker} \varphi_{*}$.

Lemma 7.4. (a) If the surface $X_{n}=X_{n, f, g}$ is smooth resp. irreducible resp. $\mathbf{Q}$ - acyclic, then so is $X_{1}=X_{1, f, g}$.

(b) Suppose that the surface $X_{n}$ where $n>1$, is smooth and irreducible, and $H_{1}\left(X_{n} ; \mathbf{Z}\right)=$ 0 . Then also $H_{1}\left(X_{1} ; \mathbf{Z}\right)=0$, and the divisor $D_{g}$ is irreducible. Hence, if $X_{n}$ is acyclic, then so is $X_{1}$.

Proof. (a) The first two statements of (a) easily follow from Lemma 7.2. As for the third one, consider the cyclic ramified covering $\mathbf{C}^{3} \ni(x, y, z) \longmapsto\left(x, y, z^{n}\right) \in \mathbf{C}^{3}$, which restricts to a cyclic covering $\sigma_{n}: X_{n} \rightarrow X_{1}$ branched to order $n$ over the curve $D_{g}^{\prime}=\{z=0\}$ in $X_{1}$. For any prime $p$ which does not divide $n$ the transfer provides an isomorphism of the homology group $H_{*}\left(X_{1} ; \mathbf{Z}_{p}\right)$ and the subgroup of the homology group $H_{*}\left(X_{n} ; \mathbf{Z}_{p}\right)$ fixed by the monodromy action [Bre, III(2.4)]. Thus, $\mathbf{Z}_{p}-$ acyclicity of $X_{n}$ implies $\mathbf{Z}_{p}$-acyclicity of $X_{1}$. If $X_{n}$ is $\mathbf{Q}$-acyclic, then it is $\mathbf{Z}_{p}$ - acyclic for all but finite number of the primes $p$, and the same holds for $X_{1}$. Therefore, $X_{1}$ is $\mathrm{Q}-$ acyclic, too. This proves (a). 
(b) Let $D_{g}^{\prime}$ resp. $D_{g}^{\prime \prime}$ be the divisor in $X_{1}$ resp. in $X_{n}$ given by the equation $z=0$. Set $X_{1}^{*}=X_{1} \backslash D_{g}^{\prime}$ and $X_{n}^{*}=X_{n} \backslash D_{g}^{\prime \prime}$. Then $\sigma_{n} \mid X_{n}^{*}: X_{n}^{*} \rightarrow X_{1}^{*}$ is an $n$-sheeted unramified covering, and hence, $\left(\sigma_{n}\right)_{*}\left(H_{1}\left(X_{n}^{*} ; \mathbf{Z}\right)\right)$ is a subgroup of index at most $n$ of the group $H_{1}\left(X_{1}^{*} ; \mathbf{Z}\right)$.

Let $\alpha\left(D_{i}^{\prime}\right) \in H_{1}\left(X_{1}^{*} ; \mathbf{Z}\right)$ resp. $\alpha\left(D_{i}^{\prime \prime}\right) \in H_{1}\left(X_{n}^{*} ; \mathbf{Z}\right)$ be the vanishing loop class of the irreducible component $D_{i}^{\prime}$ of $D_{g}^{\prime}$ resp. $D_{i}^{\prime \prime}$ of $D_{g}^{\prime \prime}, i=1, \ldots, s$. By Lemma 7.3, we have the following commutative diagram where the horizontal lines are exact sequences:

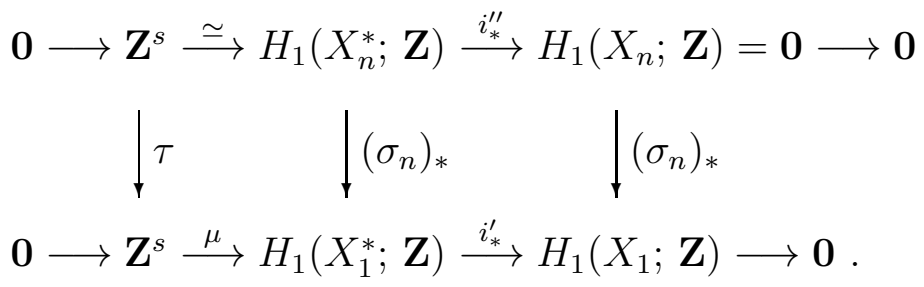

Clearly, $\left(\sigma_{n}\right)_{*}\left(\alpha\left(D_{i}^{\prime \prime}\right)\right)=n \alpha\left(D_{i}^{\prime}\right)$, and so, $\tau\left(e_{i}\right)=n e_{i}, i=1, \ldots, s$. Thus, the image $\left(\sigma_{n}\right)_{*}\left(H_{1}\left(X_{n}^{*} ; \mathbf{Z}\right)\right)$ is a subgroup of index at least $n^{s}$ of the group ker $i_{*}^{\prime} \subset H_{1}\left(X_{1}^{*} ; \mathbf{Z}\right)$. On the other hand, it should be a subgroup of index at most $n$. Therefore, $s=1$, i.e. $D_{g}$ is irreducible, and, furthermore, ker $i_{*}^{\prime}=H_{1}\left(X_{1}^{*} ; \mathbf{Z}\right)$. Hence, $H_{1}\left(X_{1} ; \mathbf{Z}\right)=0$.

The last statement of (b) follows from (a) and Lemma 7.1(a).

Lemma 7.5. If the surface $X_{n}=X_{n, f, g}$ is smooth, irreducible and $\mathbf{Q}$-acyclic, then the following assertions hold.

(a) $e\left(D_{f} \backslash D_{g}\right)=0$, or, which is equivalent, $e\left(D_{f}\right)=\kappa:=e\left(D_{f} \cap D_{g}\right)$.

Furthermore, if $n>1$, then $e\left(D_{g}\right)=e\left(D_{f} \cup D_{g}\right)=1$. If, in addition, $H_{1}\left(X_{n} ; \mathbf{Z}\right)=0$, then the divisor $D_{g}$ is smooth, reduced, irreducible and isomorphic to $\mathbf{C}$.

(b) The irreducible components $D_{f}^{(i)}, i=1, \ldots, k$, of the divisor $D_{f}$ are disjoint simply connected curves smooth outside of $D_{g}$; each of them meets supp $D_{g}$ at one point, which is smooth and reduced on $D_{g}$. In particular, $k=e\left(D_{f}\right)=e\left(D_{f} \cap D_{g}\right)=\kappa \geq 1$.

If, in addition, $H_{1}\left(X_{1} ; \mathbf{Z}\right)=0$, then each of the curves $D_{f}^{(i)}, i=1, \ldots, k$, is smooth and meets supp $D_{g}$ transversally.

Proof. (a) Denote by $E^{\prime \prime}$ resp. by $D_{g}^{\prime \prime}$ the curve in $X_{n}$ given by the equations $f=g=0$ resp. $z=0$. Consider the disjoint constructive decompositions

$$
X:=X_{n}=X^{\prime} \cup X^{\prime \prime} \cup X^{\prime \prime \prime} \quad \text { and } \quad Y:=\mathbf{C}^{2}=Y^{\prime} \cup Y^{\prime \prime} \cup Y^{\prime \prime \prime},
$$

where

$$
X^{\prime}=X \backslash\left(E^{\prime \prime} \cup D_{g}^{\prime \prime}\right), \quad X^{\prime \prime}=D_{g}^{\prime \prime} \simeq \operatorname{supp} D_{g}, \quad X^{\prime \prime \prime}=E^{\prime \prime} \backslash D_{g}^{\prime \prime}
$$

(so, $X^{\prime \prime \prime}$ is a disjoint union of curves isomorphic to $\mathbf{C}^{*}$ ), and

$$
Y^{\prime}=\mathbf{C}^{2} \backslash\left(D_{f} \cup D_{g}\right), \quad Y^{\prime \prime}=D_{g}, \quad Y^{\prime \prime \prime}=D_{f} \backslash D_{g} .
$$

Clearly, $\sigma_{I} \mid X^{\prime}: X^{\prime} \rightarrow Y^{\prime}$ is a non-ramified $n$-sheeted covering, and hence, $e\left(X^{\prime}\right)=$ $n e\left(Y^{\prime}\right)$. By the additivity of the Euler characteristic [Du], we have

$$
1=e(X)=e\left(X^{\prime}\right)+e\left(X^{\prime \prime}\right)+e\left(X^{\prime \prime \prime}\right)=n e\left(Y^{\prime}\right)+e\left(D_{g}\right)
$$


and

$$
1=e(Y)=e\left(Y^{\prime}\right)+e\left(Y^{\prime \prime}\right)+e\left(Y^{\prime \prime \prime}\right)=e\left(Y^{\prime}\right)+e\left(D_{g}\right)+e\left(D_{f} \backslash D_{g}\right) .
$$

Subtracting (2) from (1) we obtain

$$
(n-1) e\left(Y^{\prime}\right)=e\left(D_{f} \backslash D_{g}\right)=e\left(D_{f}\right)-\kappa .
$$

Putting here $n=1$ we obtain the equalities in (a). Since by Lemma 7.4(a), the surface $X_{1}:=X_{1, f, g}$ is still $\mathbf{Q}$ - acyclic, the case $n>1$ can be reduced to the case $n=1$.

Now, if $n>1$ we obtain from (3) the equality $e\left(Y^{\prime}\right)=0$. By the definition of $Y^{\prime}$, this yields the equality $e\left(D_{f} \cup D_{g}\right)=1$, and also, by $(1)$, we have $e\left(D_{g}\right)=1$.

If, in addition, $H_{1}\left(X_{n} ; \mathbf{Z}\right)=0$, then by Lemmas 7.2 and $7.4(\mathrm{~b})$, the divisor $D_{g}$ is smooth, reduced and irreducible. Since $e\left(D_{g}\right)=1$ it is isomorphic to C. This proves (a).

(b) is proven in Claims 1-6 below.

Claim 1. Each irreducible component of $D_{f}$ meets supp $D_{g}$.

Proof. Assuming that there exists an irreducible component of $D_{f}$ which does not meet supp $D_{g}$ we would get a decomposition $f=f_{1} f_{2}$ where $f_{1} \neq$ const and $\left(f_{1}, g\right)=\mathbf{C}^{[2]}$. Let $\xi, \eta \in \mathbf{C}^{[2]}$ be polynomials such that $\xi f_{1}+\eta g=1$. Replacing $\eta g=1-\xi f_{1}$ we obtain the relation $f_{1}\left(\xi+\eta f_{2} z^{n}\right) \mid X=1$, i.e. $f_{1} \mid X \in \mathbf{C}[X]$ is a non-constant invertible regular function, which is impossible. Indeed, since $b_{1}(X)=0$ the regular function $f_{1} \mid X$ can be expressed as $\exp (\psi)$ where $\psi$ is a non-constant holomorphic function on $X$. But then $f_{1} \mid X$ cannot be regular, a contradiction.

Claim 2. Each connected component of the curve supp $D_{f}$ is simply connected and meets $D_{g}$ at one point only.

Proof. Let $D_{1}, \ldots, D_{s}$ be the connected components of the curve $\operatorname{supp} D_{f}$. For a connected affine curve $\Gamma$ we always have $e(\Gamma) \leq 1$, and $e(\Gamma)=1$ iff $b_{1}(\Gamma)=0$, i.e. iff $\Gamma$ is simply connected (see e.g. [Za 1]). From this observation and Claim 1 it follows that $e\left(D_{i} \backslash D_{g}\right) \leq 0, i=1, \ldots, s$. Since we have by (a),

$$
0=e\left(D_{f} \backslash D_{g}\right)=\sum_{i=1}^{s} e\left(D_{i} \backslash D_{g}\right),
$$

we obtain that all the summands in the latter sum vanish. Thus, $0=e\left(D_{i} \backslash D_{g}\right)=$ $e\left(D_{i}\right)-e\left(D_{i} \cap D_{g}\right)$. Together with Claim 1 and the above observation this yields the inequalities $1 \leq e\left(D_{i} \cap D_{g}\right)=e\left(D_{i}\right) \leq 1$. Therefore, $e\left(D_{i} \cap D_{g}\right)=e\left(D_{i}\right)=1$, and thus $b_{1}\left(D_{i}\right)=0$, i.e. $D_{i}$ is simply connected and meets $D_{g}$ at one point only, $i=1, \ldots, s$.

Claim 3. $k \leq \kappa$ where $k$ is the number of irreducible components of $D_{f}$.

Proof. Denote $D_{f}^{(i)}, i=1, \ldots, k$, resp. $D_{g}^{(j)}, j=1, \ldots, l$, the irreducible components of the divisor $D_{f}$ resp. $D_{g}$. In the notation as in the proof of (a) above, consider the non-ramified $n$-sheeted covering $\sigma:=\sigma_{I} \mid X^{\prime}: X^{\prime} \rightarrow Y^{\prime}$. It is easily seen that $H_{1}\left(Y^{\prime} ; \mathbf{Z}\right) \simeq \mathbf{Z}^{k+l}$. As in the proof of Lemma 7.3 we have the exact sequence

$$
\mathbf{Z}^{\kappa+l} \longrightarrow H_{1}\left(X^{\prime}, \mathbf{Z}\right) \longrightarrow H_{1}(X, \mathbf{Z}) \longrightarrow \mathbf{0}
$$


Since $H_{1}(X, \mathbf{Z})$ is a torsion group it follows that $H_{1}\left(X^{\prime}, \mathbf{Z}\right)$ contains a subgroup of a finite index which is a homomorphic image of $\mathbf{Z}^{\kappa+l}$. The image $\sigma_{*}\left(H_{1}\left(X^{\prime} ; \mathbf{Z}\right)\right)$ is a finite index subgroup of the group $H_{1}\left(Y^{\prime} ; \mathbf{Z}\right)$. Henceforth, $\kappa+l \geq k+l$, or $\kappa \geq k$.

Claim 4. $k=\kappa$, and the connected components of the divisor $D_{f}$ coincide with its irreducible components.

Proof. Indeed, by (a) and Claims 2, 3 we have $\kappa=e\left(D_{f}\right)=s \leq k \leq \kappa$. Hence, $s=k=\kappa$, and the claim follows.

Claim 5. $D_{f} \backslash D_{g}$ is a smooth divisor. Furthermore, if $H_{1}\left(X_{1} ; \mathbf{Z}\right)=0$, then the divisor $D_{f}$ is smooth.

Proof. Let $D=D_{f}^{(i)}$ be an irreducible component of the divisor $D_{f}$. It is simply connected and meets $D_{g}$ at a unique point, say, $P$. Let $\nu: \mathbf{C} \rightarrow D$ be a normalization map such that $\nu(0)=P$. Then the polynomial $r:=g \circ \nu \in \mathbf{C}^{[1]}$ vanishes only at zero. This implies that the curve $D \backslash\{P\}$ is smooth (indeed, as $r(t)=c t^{m}$ for some $c \in \mathbf{C}^{*}$ and $m \in \mathbf{N}$, the derivative $r^{\prime}$ vanishes only at the origin).

It remains to show that $D$ is smooth at $P$ providing that $H_{1}\left(X_{1} ; \mathbf{Z}\right)=0$. Assume on the contrary that $P$ is a singular point of $D$. Denote $A_{1}=\mathbf{C}\left[X_{1}\right]$. Set $\varphi=f_{1} \circ \sigma_{I} \in A_{1}$ where $f_{1} \in \mathbf{C}^{[2]}$ is an irreducible polynomial which defines $D$, and $\sigma_{I}: X_{1} \rightarrow \mathbf{C}^{2}, I=$ $(f, g) \subset \mathbf{C}^{[2]}$, is the blowup morphism. Then $\varphi^{-1}(0)=\sigma_{I}^{-1}(P)=: E_{P}^{\prime}$ is the irreducible component over $P$ of the exceptional divisor $E^{\prime} \subset X_{1}$. We claim that $E_{P}^{\prime}$ is a multiple fibre of $\varphi$ whereas its generic fibres are irreducible. Indeed, since the generic fibres of $f_{1} \mid\left(\mathbf{C}^{2} \backslash D_{f}\right)$ are irreducible, in view of the isomorphism $\sigma_{I} \mid\left(X_{1} \backslash E^{\prime}\right): X_{1} \backslash E^{\prime} \stackrel{\simeq}{\longrightarrow}$ $\mathbf{C}^{2} \backslash D_{f}$ the same is true for $\varphi \mid\left(X_{1} \backslash E^{\prime}\right)$. Further, we have the equalities

$$
\operatorname{grad}\left(f_{1} \circ \pi\right) \mid E_{P}^{\prime}=\left(\operatorname{grad}_{P} f_{1}, 0\right)=\overline{0},
$$

where $\pi: \mathbf{C}^{3} \rightarrow \mathbf{C}^{2}, \pi(x, y, z)=(x, y)$. It follows that $(\operatorname{grad} \varphi) \mid E_{P}^{\prime}=0$ in local coordinates in $X_{1}$.

By Lemma 7.4(a), the surface $X_{1}$ is $\mathbf{Q}$ - acyclic. Since $H_{1}\left(X_{1} ; \mathbf{Z}\right)=0$, by Lemma 7.1, actually it is acyclic, and the algebra $A_{1}$ is UFD. Thus, in view of $\varphi^{*}(0)=m E_{P}^{\prime}$, where $m>1$, we have that $\varphi=\varphi_{1}^{m}$ for a certain $\varphi_{1} \in A_{1}$. Therefore, the generic fibres of $\varphi$ cannot be irreducible, which is a contradiction.

Claim 6. If $H_{1}\left(X_{1} ; \mathbf{Z}\right)=0$, then the curves supp $D_{f}$ and supp $D_{g}$ meet transversally. Proof. We keep all the notation from the proof of Claim 5. Assume that an irreducible component $D:=\operatorname{supp} D_{f}^{(i)}$ is tangent to $D_{g}$ at their unique intersection point $P$. By Lemma 7.2, the divisor $D_{g}$ is smooth and reduced at $P$, and $D_{f}=m D+\ldots$ for some $m>1$. Thus, we have $f=f_{1}^{m} f_{2}$ where $f_{1} \in \mathbf{C}^{[2]}$ is an irreducible polynomial which defines $D$. As above, the generic fibres of the regular function $\varphi=f_{1} \circ \pi \mid X_{1} \in A_{1}=$ $\mathbf{C}\left[X_{1}\right]$ are irreducible. We claim that $E_{P}^{\prime}:=\varphi^{-1}(0)$ is a multiple fibre of $\varphi$, which contradicts to the fact that $A_{1}$ is UFD (see the proof of Claim 5).

Indeed, by our assumption, we have $\operatorname{grad}_{P} f=\gamma \operatorname{grad}_{P} g$ for some $\gamma \in \mathbf{C}$. This implies that $(\operatorname{grad} \varphi) \mid E_{P}^{\prime}=0$ in local coordinates in $X$, or, which is the same, that $\operatorname{grad}\left(f_{1} \circ \pi\right) \mid E_{P}^{\prime}$ is proportional to $(\operatorname{grad} F) \mid E_{P}^{\prime}$ where $F:=f_{1}^{m} f_{2} z-g \in \mathbf{C}^{[3]}$ is the defining polynomial of the surface $X_{1}$ in $\mathbf{C}^{3}$. The latter follows from the equalities

$$
(\operatorname{grad} F)\left|E_{P}^{\prime}=\left(m z f_{1}^{m-1} f_{2} \operatorname{grad} f_{1}+z f_{1}^{m} \operatorname{grad} f_{2}-\operatorname{grad} g, f_{1}^{m} f_{2}\right)\right| E_{P}^{\prime}
$$




$$
=\left(-\operatorname{grad}_{P} g, 0\right)=\left(-\gamma \operatorname{grad}_{P} f_{1}, 0\right)=-\gamma \operatorname{grad}\left(f_{1} \circ \pi\right) \mid E_{P}^{\prime} .
$$

Henceforth, $(\operatorname{grad} \varphi) \mid E_{P}^{\prime}=0$, as claimed.

Now the proof of (b) is completed.

Lemma 7.6. (a) Suppose that the surface $X_{n}$ where $n>1$, is smooth and irreducible. Then the implication $(i) \Longrightarrow(v)$ of Theorem 7.2 holds.

(b) Suppose further that the surface $X_{1}$ is smooth, irreducible and $\mathbf{Q}$ - acyclic. Assume also that the curves supp $D_{f}$ and supp $D_{g}$ meet transversall21. Then, by an appropriate automorphism $\alpha^{\prime}=(\alpha$, id $) \in \operatorname{Aut}\left(\mathbf{C}^{2} \times \mathbf{C}\right)$ of $\mathbf{C}^{3}$, the equation of $X$ can be reduced to the following form:

$$
p(x) z=y+x h(x, y)+\text { const. }
$$

Proof. (a) If the condition ( $i$ ) of Theorem 7.2 is fulfilled then by Lemmas 7.1(a) and 7.4(b), both surfaces $X_{1}$ and $X_{n}$ are acyclic. Thus, by Lemma 7.5, $D_{g} \simeq \mathbf{C}$ and $D_{f}^{(i)} \simeq \mathbf{C}$ for each $i=1, \ldots, \kappa$.

Let $f_{1} \in \mathbf{C}^{[2]}$ be an irreducible polynomial which defines the irreducible component $D_{f}^{(1)}$ of $D_{f}$. Since by Lemma 7.5(b), the components $D_{f}^{(i)}, i=1, \ldots, \kappa$, of $D_{f}$ are disjoint and simply connected, for each $i=1, \ldots, \kappa$ we have $f_{1} \mid D_{f}^{(i)} \equiv$ const $=c_{i}$, and therefore, $f=p\left(f_{1}\right)$ for some polynomial $p \in \mathbf{C}^{[1]}$. In view of Lemma 7.5(b), the curves $D_{f}^{(1)} \simeq \mathbf{C}$ and $D_{g} \simeq \mathbf{C}$ meet transversally at a unique point. By the Abhyankar-Moh and Suzuki Embedding Theorem, it follows that $\alpha:=\left(f_{1}, g\right): \mathbf{C}^{2} \rightarrow \mathbf{C}^{2}$ is an automorphism which transforms the pair $\left(f_{1}, g\right)$ into a pair of coordinate functions $(x, y)$, and the pair $(f, g)$ into the pair $(p(x), y)$, as desired. This proves the implication $(i) \Longrightarrow(v)$ of Theorem 7.2 .

(b) By the Abhyankar-Moh and Suzuki Embedding Theorem, the smooth simply connected curve $D_{f}^{(1)} \simeq \mathbf{C}$ can be transformed into a coordinate line $x=0$ by an automorphism of $\mathbf{C}^{2}$. Thus, we may assume that $f_{1}(x, y)=x$, and so, as above, $f(x, y)=p(x)$ and $X=\{p(x) z=g(x, y)\} \subset \mathbf{C}^{3}$.

If $x_{i}$ is a root of $p$, that is, $D_{f}^{(i)}=\left\{x=x_{i}\right\}$, then by Lemma 7.5(b), the polynomial $g\left(x_{i}, y\right) \in \mathbf{C}[y]$ has only one root, say, $c_{i}$, and this root is simple, that is, $g\left(x_{i}, y\right)=$ $\gamma_{i}\left(y-c_{i}\right)$. Hence, $g(x, y)=\gamma_{i}\left(y-c_{i}\right)+\left(x-x_{i}\right) h_{i}(x, y)$ for a certain $h_{i} \in \mathbf{C}^{[2]}$. Plugging here $x_{1}=0$ and replacing $z$ by $z / \gamma_{1}$, we obtain the desired presentation.

This lemma and the next proposition yield the implication $(i) \Longrightarrow(i v)$ of Theorem 7.2 for $n=1$. Denote by $G$ the subgroup of the group Aut $\mathbf{C}^{3}$ which consists of the automorphisms of the type $(x, y, z) \longmapsto\left(x, \gamma_{1} y+x g_{1}, \gamma_{2} z+x g_{2}\right)$ where $g_{i} \in \mathbf{C}^{[3]}$ and $\gamma_{i} \in \mathbf{C}^{*}, i=1,2$.

Proposition 7.1. Let $X$ be an irreducible smooth $\mathbf{Q}$-acyclic surface given in $\mathbf{C}^{3}$ by the equation $p(x) z=g(x, y)$ where $p \in \mathbf{C}^{[1]} \backslash \mathbf{C}$ and $g \in \mathbf{C}^{[2]} \backslash \mathbf{C}$. Suppose that the curves supp $D_{p}$ and supp $D_{g}$ meet transversally22. Then $X$ can be transformed into a plane $L_{c}:=\{y=c\}, c \in \mathbf{C}$, by an automorphism $\alpha \in G$.

The proof proceeds by induction on $\operatorname{deg} p$.

Claim 1. The statement is true if $\operatorname{deg} p=1$.

\footnotetext{
${ }^{21}$ In particular, as follows from Lemma 7.5(b), supp $D_{f}$ is smooth.

${ }^{22}$ By Lemma 7.5 (b), this is true if $X$ satisfies the condition $(i)$ of Theorem 7.2 .
} 
Proof. We may assume that $p(x)=x$, and by Lemma 7.5(b), that $g(x, y)=y+$ $x h(x, y)-c, c \in \mathbf{C}$. Then the automorphism $\alpha \in \operatorname{Aut} \mathbf{C}^{3}, \alpha(x, y, z)=(x, y, z+h)$, transforms $X$ into the surface $X^{\prime}:=\{x z=y-c\}$. Furthermore, the automorphism $\beta \in G, \beta(x, y, z)=(x, y+x z, z)$, transforms $X^{\prime}$ into the plane $L_{c}$. The resulting automorphism $\alpha^{\prime}:=\beta \circ \alpha, \alpha^{\prime}(x, y, z)=\left(x, y+x\left(z+h_{1}\right), z+h_{1}\right)$ where $h_{1}=h(x, y+$ $x z)$, does not belong, in general, to the group $G$. But composing it further with the automorphism $\beta^{\prime} \in$ Aut $\mathbf{C}^{3}, \beta^{\prime}(x, y, z)=\left(x, y, z-h_{1}(0, y)\right)$, we obtain a new one $\alpha^{\prime \prime}=\beta^{\prime} \circ \alpha^{\prime}$ which does belong to $G$. It remains to note that $\beta^{\prime}$ preserves the plane $L_{c}$, hence, $\alpha^{\prime \prime}(X)=\alpha^{\prime}(X)=L_{c}$, and we are done.

Claim 2. Let $p(x)=x q(x)$ where $\operatorname{deg} q \geq 1$. Consider the surface $Y=\{q(x) z=$ $g(x, y)\}$ in $\mathbf{C}^{3}$. Then $X=\Sigma_{I_{1}, \xi}(Y)$ is the affine modification of $Y$ along the divisor $D_{\xi}$ with center $I_{1}=(\xi, \eta) \subset \mathbf{C}[Y]$ where $\xi=x \mid Y$ and $\eta=z \mid Y$. Furthermore, $Y$ is a smooth irreducible $\mathbf{Q}$ - acyclic surface; it is acyclic if $X$ is.

Proof. The modification $\Sigma_{I, x}\left(\mathbf{C}^{3}\right)$ along the plane $D_{x}=\{x=0\}$ with center $I:=$ $(x, z) \subset \mathbf{C}^{[3]}$ is isomorphic to $\mathbf{C}^{3}$, and the blowup morphism $\sigma_{I}: \mathbf{C}^{3} \rightarrow \mathbf{C}^{3}$ is given as $(x, y, z) \longmapsto(x, y, x z)$ (cf. Examples 1.3, 2.1). By Proposition 2.2(b), the strict transform $X=Y^{\prime}$ of $Y$ under $\sigma_{I}$ coincides with the modification $\Sigma_{I_{1}, \xi}(Y)$. This proves the first assertion.

It is easily seen that under our assumptions, $X$ being smooth and irreducible implies the same for $Y$ (cf. Lemma 7.2).

Due to our assumptions and to Lemma 7.5(b), the irreducible component $D_{p}^{1}:=$ $\{x=0\}$ of the divisor $D_{p}=p^{*}(0)$ in $\mathbf{C}^{2}$ meets the divisor $D_{g}$ transversally at one point, say, $P_{0}=\left(0, y_{0}\right)$, and $D_{g}$ is reduced at $P_{0}$. Thus, we have $g(0, y)=\gamma\left(y-y_{0}\right)$. Hence, the polynomials $x, g(0, y), z \in \mathbf{C}^{[3]}$ generate the maximal ideal of the point $P_{0}^{\prime}:=\left(0, y_{0}, 0\right) \in Y \subset \mathbf{C}^{3}$. It follows that $I_{1}=(\xi, \eta)=I\left(P_{0}^{\prime}\right)$ is a maximal ideal of the algebra $\mathbf{C}[Y]$. Thereby, the blowup morphism $\sigma: X \rightarrow Y, \sigma:=\sigma_{I_{1}}=\sigma_{I} \mid X$, consists of the usual blow up at $P_{0}^{\prime}$ and deleting the strict transform of the curve $l_{0}:=$ $\{\xi=0\} \subset Y, l_{0} \simeq \mathbf{C}$, passing through $P_{0}^{\prime}$ (see Proposition 1.1). By Theorem 3.1 (see also [Ka 1, Thm. 3.5], [Za 2, Thm. 5.1]), this modification preserves the homology, i.e. $\sigma_{*}: H_{*}(X ; \mathbf{Z}) \longrightarrow H_{*}(Y ; \mathbf{Z})$ is an isomorphism. Thus, $Y$ is $\mathbf{Q}-$ acyclic resp. acyclic iff $X$ is.

Claim 3: The induction step. Let $Y$ be as in Claim 2. Suppose that $Y$ can be transformed into a plane $L_{c}:=\{y=c\}, c \in \mathbf{C}$, by an automorphism $\alpha \in G$. Then the same is true for $X$.

Proof. Notice that the action of the group $G$ on $\mathbf{C}^{[3]}$ preserves the ideal $I=(x, z)$ and fixes the element $x \in I$. Therefore, by Corollary 2.2, $\alpha$ can be lifted in a unique way to an automorphism $\alpha^{\prime}$ of $\mathbf{C}^{3}=\Sigma_{I, x}\left(\mathbf{C}^{3}\right)$ such that $\alpha \circ \sigma_{I}=\sigma_{I} \circ \alpha^{\prime}$. The automorphism $\alpha^{\prime}$ is of the form

$$
\alpha^{\prime}:(x, y, z) \longmapsto\left(x, \gamma_{1} y+x g_{1}(x, y, x z), \gamma_{2} z+g_{2}(x, y, x z)\right)
$$

(see Example 2.3). It sends the surface $X=Y^{\prime}$ onto the strict transform $L_{c}^{\prime}=L_{c}$ of the plane $L_{c}$, i.e. again onto the plane $L_{c}$ (indeed, $\left.\sigma_{I}^{*}(y)=y\right)$. Now, composing $\alpha^{\prime}$ with the automorphism $\beta \in$ Aut $\mathbf{C}^{3}, \beta(x, y, z)=\left(x, y, z-g_{2}\left(0, \gamma_{1}^{-1} y, 0\right)\right)$, we get an automorphism $\alpha^{\prime \prime}:=\beta \circ \alpha^{\prime}$ which, as it can be easily seen, belongs to the group $G$. 
Since $\beta$ preserves the plane $L_{c}, \alpha^{\prime \prime}$ still transforms $X$ into this plane. This proves Claim 3 and completes the proof of the proposition.

Now the proof of Theorem 7.2 is completed. The next example shows that Theorem 7.2 cannot be extended to $\mathrm{Q}$ - acyclic surfaces.

Example 7.1: A Q-acyclic modification of the plane. Consider the surface $X \subset \mathbf{C}^{3}$ given by the equation $x^{2} z=x+y^{2}$. It is easily seen that $X$ is $\mathbf{Q}-$ acyclic but not acyclic. Indeed, in the notation as in Proposition 7.1 we have $E^{\prime} \simeq \mathbf{C}, \quad X \backslash E^{\prime} \simeq \mathbf{C}^{*} \times \mathbf{C}$ and $\sigma^{*}\left(D_{p}\right)=2 E^{\prime}$, which provides that $H_{i}(X ; \mathbf{Z})=0$ for $i \geq 2$ and $H_{1}(X ; \mathbf{Z}) \simeq \mathbf{Z} / 2 \mathbf{Z}$ (cf. the proof of Theorem 3.1). Thus, the assumption of Proposition 7.1 that the curves $\operatorname{supp} D_{p}$ and $\operatorname{supp} D_{g}$ meet transversally is essential, as well as the condition $E^{\prime}=\sigma^{*}(D)$ in (i) of Theorem 3.1.

\section{References}

[AM] S.S. Abhyankar, T.T. Moh, Embedding of the line in the plane, J. Reine Angew. Math. 276 (1975), 148-166.

[Akh] D.N. Akhiezer, Lie group actions in complex analysis, Prospects in Mathem., Vieweg, 1995.

[As] T. Asanuma, Non-linearizable algebraic $k^{*}$-actions on affine spaces, preprint, 1996, $23 \mathrm{p}$.

[Bre] G.E.Bredon, Introduction to compact transformation groups, Academic Press, N.Y., 1972.

[Bri] E. Brieskorn, Beispiele zur Differentialtopologie von Singularitäten, Invent. Math. 2 (1966), 1-14.

[Da] E.D. Davis, Ideals of the principal class, $R$-sequences and a certain monoidal transformation, Pacific J. Math. 20 (1967), 197-205.

[De] H. Derksen, Constructive Invariant Theory and the Linearization Problem, Ph.D. thesis, Basel, 1997.

[tDP] T. tom Dieck, T. Petrie, Contractible affine surfaces of Kodaira dimension one, Japan J. Math. 16 (1990), 147-169.

[Di 1] A. Dimca, Hypersurfaces in $\mathbf{C}^{2 n}$ diffeomorphic to $\mathbf{R}^{4 n-2}(n \geq 2)$, Max-Planck Institute, preprint, 1991.

[Di 2] A. Dimca, Singularities and Topology of Hypersurfaces, Universitext, Springer, 1992.

[Do] A. Dold, Lectures on algebraic topology, Springer, Berlin e.a., 1974. 
[Du] A. Durfee, Algebraic varieties which are a disjoint union of subvarieties, In: Geometry and Topology. Proc. Conf. Athens/Ga, 1985. Lect. Notes Pure Appl. Math. 105 (1987), 99-102.

[Ei] D. Eisenbud, Commutative algebra with a view towards algebraic geometry, Graduate Texts in Math., Springer, N.Y. e.a., 1994

[FlZa] H. Flenner, M. Zaidenberg, Q-acyclic surfaces and their deformations, Contempor. Mathem. 162, Providence, RI, 1994, 143-208.

[FoFu] A.T. Fomenko, B.D. Fuks, A course in homotopic topology, Textbook, Nauka, Moscow, 1989 (Russian).

[Fuj] T. Fujita, On the topology of non-complete algebraic surfaces, J. Fac. Sci. Univ. Tokyo, Sect.IA, 29 (1982), 503-566.

[Ful] W. Fulton, Intersection Theory, Ergebnisse der Mathematik, B.2, Springer, Berlin e.a., 1984.

[Ha] R. Hartshorn, Algebraic geometry, Springer, NY e.a., 1977.

[Hiro] H. Hironaka, Resolution of singularities, Ann. Math. 79 (1964), 109-326.

[Je] Z. Jelonek, The extension of regular and rational embeddings, Math. Ann. 113 (1987) 113-120.

[Ju] H. W. E. Jung, Über ganze birationale Transformationen der Ebene, J. reine und angew. Math., 184 (1942), 161-174.

[Ka 1] S. Kaliman, Exotic analytic structures and Eisenman intrinsic measures, Israel Math. J. 88 (1994), 411-423.

[Ka 2] S. Kaliman, Extensions of isomorphisms between affine algebraic subvarieties of $k^{n}$ to automorphisms of $k^{n}$, Proc. Amer. Math. Soc. 113 (1991), 325-334.

[Ka 3] S. Kaliman, Smooth contractible hypersurfaces in $\mathbf{C}^{n}$ and exotic algebraic structures on $\mathbf{C}^{3}$, Math. Zeitschrift 214 (1993), 499-510.

[KaML 1] S. Kaliman, L. Makar-Limanov, On Russell-Koras contractible threefolds, J. of Algebraic Geom. 6 (1997), 247-268.

[KaML 2] S. Kaliman, L. Makar-Limanov, Affine algebraic manifolds without dominant morphisms from Euclidean spaces, Rocky Mount. J. Math. 27:2 (1997), 601 -609.

[ML 1] L. Makar-Limanov, On the hypersurface $x+x^{2} y+z^{2}+t^{3}=0$ in $\mathbf{C}^{4}$ or a $\mathbf{C}^{3}$-like threefold which is not $\mathbf{C}^{3}$, Israel J. Math. 96 (1996), 419-429.

[ML 2] L. Makar-Limanov, On groups of automorphisms of a class of surfaces, Israel J. Math. 69 (1990), 250-256. 
[Mik] A. Micali, Sur les algèbres universalles, Annales Inst. Fourier, Grenoble 14 (1964), $33-88$.

[Mil 1] J. Milnor, Morse Theory, Princeton Univ. Press, Princeton, NJ, 1963.

[Mil 2] J. Milnor, On the 3-dimensional Brieskorn manifolds $M(p, q, r)$, in: Knots, groups, and 3-manifolds, L. P. Neuwirth, ed. Annals of Math. Stud., Princeton Univ. Press, Princeton, NJ, 1975, 175-225.

[MilSta] J. Milnor, J. Stasheff, Characteristic classes, Annals of Mathem. Studies 76, Princeton Univ. Press and Univ. of Tokyo Press, Princeton, NJ, 1974.

[Miy] M. Miyanishi, Algebraic characterization of the affine 3-space, Proc. Algebraic Geom. Seminar, Singapore, World Scientific, 1987, 53-67.

[Ram] C.P. Ramanujam, A topological characterization of the affine plane as an algebraic variety, Ann. Math., 94 (1971), 69-88.

[Re] R. Rentschler, Opérations du groupe additif sur le plane affine, C.R. Acad. Sci. Paris, 267 (1968), 384-387.

[RoRu] J.-P. Rosay, W. Rudin, Holomorphic maps from $\mathbf{C}^{n}$ to $\mathbf{C}^{n}$, Trans. Amer. Math. Soc. 310 (1988), 47-86.

[Ru 1] P. Russell, On a class of $\mathbf{C}^{3}$-like threefolds, Preliminary Report, Berlin, 1992.

[Ru 2] P. Russell, Simple birational extensions of two dimensional affine rational domains, Compos. Math. 33 (1976), 197-208.

[RuSat] P. Russell, A. Sathaye, On finding and cancelling variables in $k[X, Y, Z], \mathrm{J}$. Algebra 57 (1979), 151-166.

[Sat] A. Sathaye, On linear planes, Proc. Amer. Math. Soc. 56 (1976), 1-7.

[Se] J.-P. Serre, Trees, Springer Verlag, Berlin-Heidelberg-New York, 1980.

[Sr] V. Srinivas, On the embedding dimension of an affine variety, Math. Ann. 289 (1991), 125-132.

[Suz] M. Suzuki, Propiétés topologiques des polynômes de deux variables complexes, et automorphismes algébrigue de l'espace C $^{2}$, J. Math. Soc. Japan, 26 (1974), 241257.

[vdK] W. van der Kulk, On polynomial rings in two variables, Nieuw Arch. Wisk. (3) 1 (1953), 33-41.

[Va] W.V. Vasconselos, Arithmetic of blowup algebras, London Math. Soc. Lect. Notes Ser. 195, Cambridge Univ. Press, 1994.

[Wr 1] D. Wright, Abelian subgroups of $\operatorname{Aut}_{k}(k[X, Y])$ and applications to actions on the affine plane, Ill. J. Math. 23 (1979), 579-634. 
[Wr 2] D. Wright, Cancellation of variables of the form $b T^{n}-a$, J. Algebra 52 (1978), 94-100.

[Za 1] M. Zaidenberg, Rational actions of the group $\mathbf{C}^{*}$ on $\mathbf{C}^{2}$, their quasi-invariants, and algebraic curves in $\mathrm{C}^{2}$ with Euler characteristic 1, Soviet Math. Dokl. 31:1 (1985), 57-60.

[Za 2] M. Zaidenberg, Lectures on exotic algebraic structures on affine spaces, Schriftenreihe des Graduiertenkollegs Geometrie und Mathematische Physik, Heft 24, Institut Für Mathematik, Ruhr-Universität Bochum, Juli 1997, 56p.

[Zar] O. Zariski, Foundations of a general theory of birational correspondences, Trans. Amer. Math. Soc. 53 (1943), 497-542

Shulim Kaliman

Department of Mathematics and Computer Science

University of Miami

Coral Gables

FL 33124

U.S.A.

e-mail:

kaliman@math.miami.edu
Mikhail Zaidenberg

Institut Fourier de Mathématiques

UMR 5582

Université Grenoble I

BP 74

38402 St. Martin d'Hères-cédex

France

e-mail:

zaidenbe@ujf-grenoble.fr 